\title{
Herschel-HIFI observations of $\mathrm{H}_{2} \mathrm{O}, \mathrm{NH}_{3}$, and $\mathrm{N}_{2} \mathrm{H}^{+}$toward high-mass starless and protostellar clumps identified by the Hi-GAL survey ${ }^{\star}, \star \star$
}

\author{
L. Olmi ${ }^{1,2}$, C. M. Persson ${ }^{3}$, and C. Codella ${ }^{1}$ \\ 1 INAF, Osservatorio Astrofisico di Arcetri, Largo E. Fermi 5, 50125 Firenze, Italy \\ e-mail: olmi.luca@gmail.com \\ 2 University of Puerto Rico, Rio Piedras Campus, Physics Dept., Box 23343, UPR station, San Juan, Puerto Rico, USA \\ 3 Chalmers University of Technology, Department of Earth and Space Sciences, Onsala Space Observatory, 43992 Onsala, Sweden
}

Received 6 July 2015 / Accepted 25 August 2015

\begin{abstract}
Context. Our present understanding of high-mass star formation still remains very schematic. In particular, it is not yet clear how much of the difference between low-mass and high-mass star formation occurs during the earliest star formation phases.

Aims. The chemical characteristics of massive cold clumps, and the comparison with those of their low-mass counterparts, could provide crucial clues about the exact role that chemistry plays in differentiating the early phases of low-mass and high-mass star formation. Water, in particular, is a unique probe of physical and chemical conditions in star-forming regions.

Methods. Using the HIFI instrument of Herschel, we have observed the ortho- $\mathrm{NH}_{3}\left(1_{0}-0_{0}\right)(572 \mathrm{GHz})$, ortho- $\mathrm{H}_{2} \mathrm{O}\left(1_{10}-1_{01}\right)$ $(557 \mathrm{GHz})$, and $\mathrm{N}_{2} \mathrm{H}^{+}(6-5)(559 \mathrm{GHz})$ lines toward a sample of high-mass starless and protostellar clumps selected from the Herschel Infrared Galactic Plane Survey (Hi-GAL). We compare our results to previous studies of low-mass and high-mass protostellar objects. Results. At least one of the three molecular lines was detected in 4 (out of 35) and 7 (out of 17) objects in the $\ell=59^{\circ}$ and $\ell=30^{\circ}$ galactic regions, respectively. All detected sources are protostellar. The water spectra are complex and consist of several kinematic components, identified through a Gaussian decomposition, and we detected inverse and regular P-Cygni profiles in a few sources. All water line profiles of the $\ell=59^{\circ}$ region are dominated by a broad Gaussian emission feature, indicating that the bulk of the water emission arises in outflows. No such broad emission is detected toward the $\ell=30^{\circ}$ objects. The ammonia line in some cases also shows line wings and an inverse $\mathrm{P}$-Cygni profile, thus confirming that $\mathrm{NH}_{3}$ rotational transitions can be used to probe the dynamics of high-mass, star-forming regions. Both bolometric and water line luminosity increase with the continuum temperature.

Conclusions. The higher water abundance toward the $\ell=59^{\circ}$ sources, characterized by the presence of outflows and shocks, supports a scenario in which the abundance of this molecule is linked to the shocked gas. Various indicators suggest that the detected sources toward the $\ell=30^{\circ}$ region are in a somewhat later evolutionary phase compared to the $\ell=59^{\circ}$ field, although a firm conclusion is limited by the small number of observed sources. We find many similarities with studies carried out toward low-mass protostellar objects, but there are indications that the level of infall and turbulence in the high-mass protostars studied here could be significantly higher.
\end{abstract}

Key words. line: formation - stars: formation - ISM: molecules - ISM: abundances

\section{Introduction}

High-mass stars (OB spectral type, $M \gtrsim 8 M_{\odot}$ ), though much rarer than low-mass and intermediate-mass stars, are fundamental in the evolution of galaxies. While a reasonably robust evolutionary sequence has been established for the formation and early evolution of isolated low-mass stars, our present understanding of high-mass star formation still remains very schematic (Zinnecker \& Yorke 2007; Tan et al. 2014). In particular, some of the main issues regarding high-mass star formation that still need to be studied are: (i) what is the physical and dynamical evolutionary path of massive starless ${ }^{1}$

\footnotetext{
* Herschel is an ESA space observatory with science instruments provided by European-led Principal Investigator consortia and with important participation from NASA.

$\star \star$ Appendices are available in electronic form at http://www . aanda.org

1 As identified through the absence of mid-IR emission and/or the strength of the PACS $70 \mu \mathrm{m}$ emission.
}

clumps $^{2}$ toward the protostellar and stellar phases? And, (ii) how much of the difference between low-mass and high-mass star formation occurs during the earliest star formation phases?

The high-mass analogues of low-mass Class 0 protostars and starless clumps (e.g., Motte et al. 2007; Bontemps et al. 2010; Olmi et al. 2010; Olmi et al. 2013) constitute a fundamental link between the global processes that regulate star formation on large scales and the properties of newly formed (proto)stars and clusters. Therefore, to better define the evolutionary path from molecular clumps to protostars it is crucial to determine the physical and dynamical (and, possibly, chemical) properties of these early phases of high-mass star formation.

\footnotetext{
2 In the literature, the term "core" or "fragment" usually refers to small-scale objects $(\$ 0.1 \mathrm{pc}$ or even $\ll 0.1 \mathrm{pc})$, possibly corresponding to a later stage of fragmentation, while the term "clump" is generally used for a somewhat larger $(\gtrsim 0.1-1 \mathrm{pc})$, unresolved object, possibly composed of several cores. The term clump is thus more appropriate to refer to the compact objects described in this work.
} 
Table 1. Observed transitions.

\begin{tabular}{lccccc}
\hline \hline Species & $\begin{array}{c}\text { Frequency }^{a} \\
{[\mathrm{GHz}]}\end{array}$ & Transition $^{b}$ & $\begin{array}{c}E_{\mathrm{l}} \\
{[\mathrm{K}]}\end{array}$ & $\begin{array}{c}E_{\mathrm{u}} \\
{[\mathrm{K}]}\end{array}$ & $\begin{array}{c}\log \left(A_{\mathrm{ul}}\right) \\
{\left[\mathrm{s}^{-1}\right]}\end{array}$ \\
\hline${\text { ortho- } \mathrm{NH}_{3}}^{572.4982}$ & $J_{K}=1_{0}-0_{0}$ & 0 & 27 & -2.80 \\
${\text { ortho- } \mathrm{H}_{2} \mathrm{O}}^{2}$ & 556.9359 & $J_{K_{a}, K_{c}}=1_{1,0}-1_{0,1}$ & 34 & 61 & -2.46 \\
$\mathrm{~N}_{2} \mathrm{H}^{+}$ & 558.9665 & $J=6-5$ & 67 & 94 & -1.96 \\
\hline
\end{tabular}

Notes. ${ }^{(a)}$ Frequencies from http://www.cv.nrao.edu/php/splat/ (b) Critical densities are $n_{\mathrm{cr}} \gtrsim 10^{7}-10^{8} \mathrm{~cm}^{-3}$ for all three lines.

The formation of both low-mass and high-mass stars from the initial dense clumps is accompanied by large changes in the temperature and density structure in their surrounding envelopes. These changes in turn open up different chemical routes. Current observational evidence indicates that significant amounts of volatile molecules, for instance $\mathrm{CO}, \mathrm{H}_{2} \mathrm{O}$ and $\mathrm{NH}_{3}$, reside on the surfaces of dust grains in dense clumps. These surface molecules can be formed either in the gas phase via ion-molecule reactions followed by accretion onto the dust particles, or via direct production on the grain surfaces. Different physical conditions, for example during the early formation phases of low-mass and high-mass stars, could thus determine which of these different chemical routes to molecule formation are active and/or dominant.

The chemical characteristics of massive cold clumps, and the comparison with those of their low-mass counterparts, are only starting to emerge (Russeil et al. 2010; Marseille et al. 2010). While governed by the same processes as lower mass objects, the much larger luminosity of massive stars during all their evolutionary phases greatly affect the outcome. Therefore, the comparison of chemical models (e.g., with different weights of grain vs. gas chemistry) with aimed spectral line observations provides crucial clues about the exact role that chemistry plays in differentiating the early phases of high-mass star formation from those of low-mass star formation.

The $\left(1_{0}-0_{0}\right)$ ground-state rotational transition of $o-\mathrm{NH}_{3}$ (e.g., Ho \& Townes 1983) has an upper state energy of $27 \mathrm{~K}$ (see Table 1), comparable to the rotation-inversion transitions at $\mathrm{cm}$ wavelengths. The critical density is, however, four orders of magnitude higher and is thus a better probe of the physical conditions of the dense high-mass starless clumps detected by the Hi-GAL survey. Moreover, its ortho symmetry form makes it a complementary tool to the commonly used rotation-inversion transitions of para- $\mathrm{NH}_{3}$ observable from the ground. Both $\mathrm{NH}_{3}$ and $\mathrm{N}_{2} \mathrm{H}^{+}$are well-known tracers of low-mass, pre- and protostellar clumps.

Water is another important component in the chemistry of regions of star formation. In cold gas, water is mainly produced on dust grains and released to the gas phase when the temperature increases $(\gtrsim 100 \mathrm{~K})$. Water is thus not expected to be found with high abundances in cold, starless clumps (e.g., Bergin \& Tafalla 2007; Caselli et al. 2012; Wirström et al. 2014). In fact, recent results from the Herschel key program "Water in star-forming regions with Herschel" (WISH; van Dishoeck et al. 2011) have shown that during the quiescent phases of star formation, the water abundance is very low. The embedded stage of star formation, however, shows very broad and complex line profiles consisting of many dynamical components (Kristensen \& van Dishoeck 2011; Kristensen et al. 2012). The WISH team also found, comparing typical low-, intermediate-, and high-mass young stellar objects, that their spectra were remarkably similar, characterized by broad, complex line profiles and consisting of multiple components. Our observations mainly consist of high-mass clumps in various evolutionary stages, and thus we also try to identify possible evolutionary differences.

The outline of the paper is as follows. In Sect. 2 we discuss how our sources were selected and observed with Herschel. In Sect. 3 we describe how the main kinematical and physical parameters of the detected sources were derived. The gas kinematics is further discussed in Sect. 4 and we draw our conclusions in Sect. 5.

\section{Observations and data reduction}

\subsection{Source selection}

We have selected our targets from the preliminary source catalog obtained toward the two Galactic fields, at $\ell=30^{\circ}$ and $\ell=59^{\circ}$, observed for the Herschel Infrared Galactic Plane Survey (Hi-GAL, Molinari et al. 2010) during the science demonstration Phase of Herschel (Elia et al. 2010). The HiGAL (and Hi-GAL360) Project used SPIRE and PACS in parallel mode to carry out an unbiased imaging survey of the Galactic plane, uniformly sampling a $2^{\circ}$-wide strip, with $-60^{\circ}<\ell<60^{\circ}$, in the $70,170,250,350$, and $500 \mu \mathrm{m}$ photometric bands. The source identification, flux extraction, and spectral energy density building in these two Hi-GAL maps were carried out adapting to the Herschel SPIRE/PACS wavebands the methods and techniques described by Netterfield et al. (2009) and Olmi et al. (2009). From the preliminary catalogs of the $\ell=30^{\circ}$ and $\ell=59^{\circ}$ fields, we have extracted both high-mass, starless clumps and high-mass, protostellar objects.

We selected the candidate high-mass starless clumps by imposing that $M_{\text {clump }}>100 M_{\odot}\left(M_{\text {clump }}>200 M_{\odot}\right)$ and $L_{\text {fir }}<$ $100 L_{\odot}\left(L_{\mathrm{fir}}<500 L_{\odot}\right)$ for the $\ell=59^{\circ}\left(\ell=30^{\circ}\right)$ field. Moreover, we checked that no compact Multiband Imaging Photometer for Spitzer (MIPS; Rieke et al. 2004) $24 \mu \mathrm{m}$ emission were present at the position of the candidate high-mass starless clump. Instead, candidate high-mass protostellar objects do have a compact MIPS $24 \mu \mathrm{m}$ counterpart. Finally, the sources have been visually inspected to reject ambiguous detections. This procedure resulted in a total of 81 clumps, including the four candidate high-mass starless clumps already observed with the VLA and the Effelsberg 100-m telescope in the $\mathrm{NH}_{3}(1,1)$ and $(2,2)$ inversion lines by Olmi et al. (2010). Out of the initial sample, 52 sources were finally observed with Herschel: 17 objects in the $\ell=30^{\circ}$ region and 35 in the $\ell=59^{\circ}$ region. The observed sources and their basic physical parameters are listed in Table 2. We highlighted sources with a detection in at least one of the three observed lines in boldface.

\subsection{Herschel observations}

Using the HIFI instrument (de Graauw et al. 2010) of Herschel (Pilbratt et al. 2010) we have observed the $o-\mathrm{NH}_{3}\left(1_{0}-0_{0}\right)$ line at $572 \mathrm{GHz}$ in the upper side band of band $1 \mathrm{~b}$, and simultaneously in the lower side band both the $o-\mathrm{H}_{2} \mathrm{O}\left(1_{10}-1_{01}\right)$ line at $557 \mathrm{GHz}$, and the $\mathrm{N}_{2} \mathrm{H}^{+}(6-5)$ line at $559 \mathrm{GHz}$ (see Table 1). We used the wide-band spectrometer with a bandwidth of $4 \mathrm{GHz}$ and an effective spectral resolution of $1.1 \mathrm{MHz}(\Delta V=$ $\left.0.27 \mathrm{~km} \mathrm{~s}^{-1}\right)$. The half-power beam width and the main-beam efficiency at $572 \mathrm{GHz}$ are $36^{\prime \prime}$ and 0.62 , respectively (Mueller et al. $\left.2014^{3}\right)$. The total calibration uncertainties are $\simeq 9 \%$ for band $1^{4}$ (Roelfsema et al. 2012). We used the dual beam switching (DBS)

\footnotetext{
http://herschel.esac.esa.int/twiki/pub/Public/ HifiCalibrationWeb/HifiBeamReleaseNote_Sep2014.pdf 4 http://herschel. esac. esa.int/Docs/HIFI/html/ch5.html
} 
L. Olmi et al.: High-mass starless and protostellar clumps identified by the Hi-GAL survey

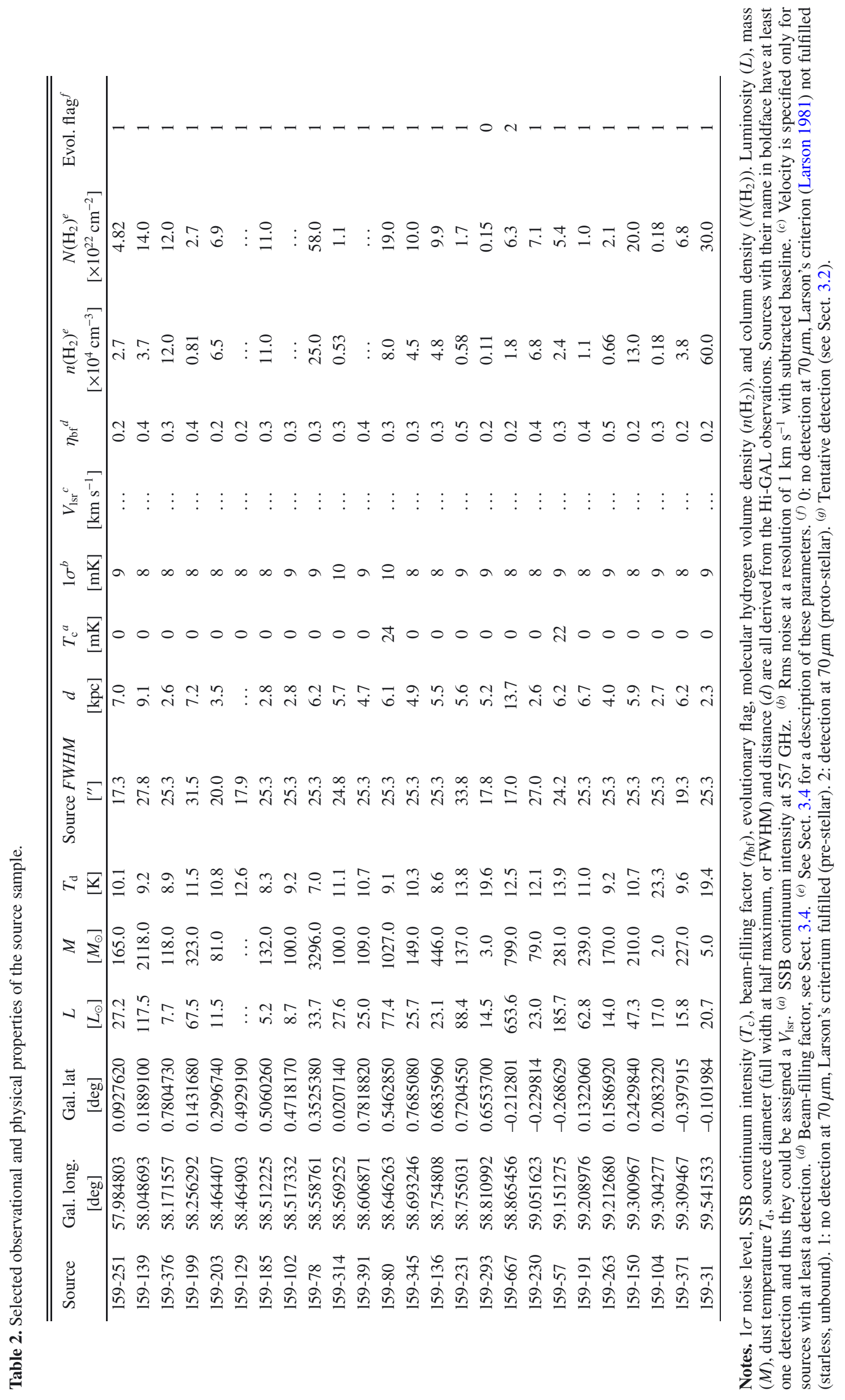

A125, page 3 of 28 


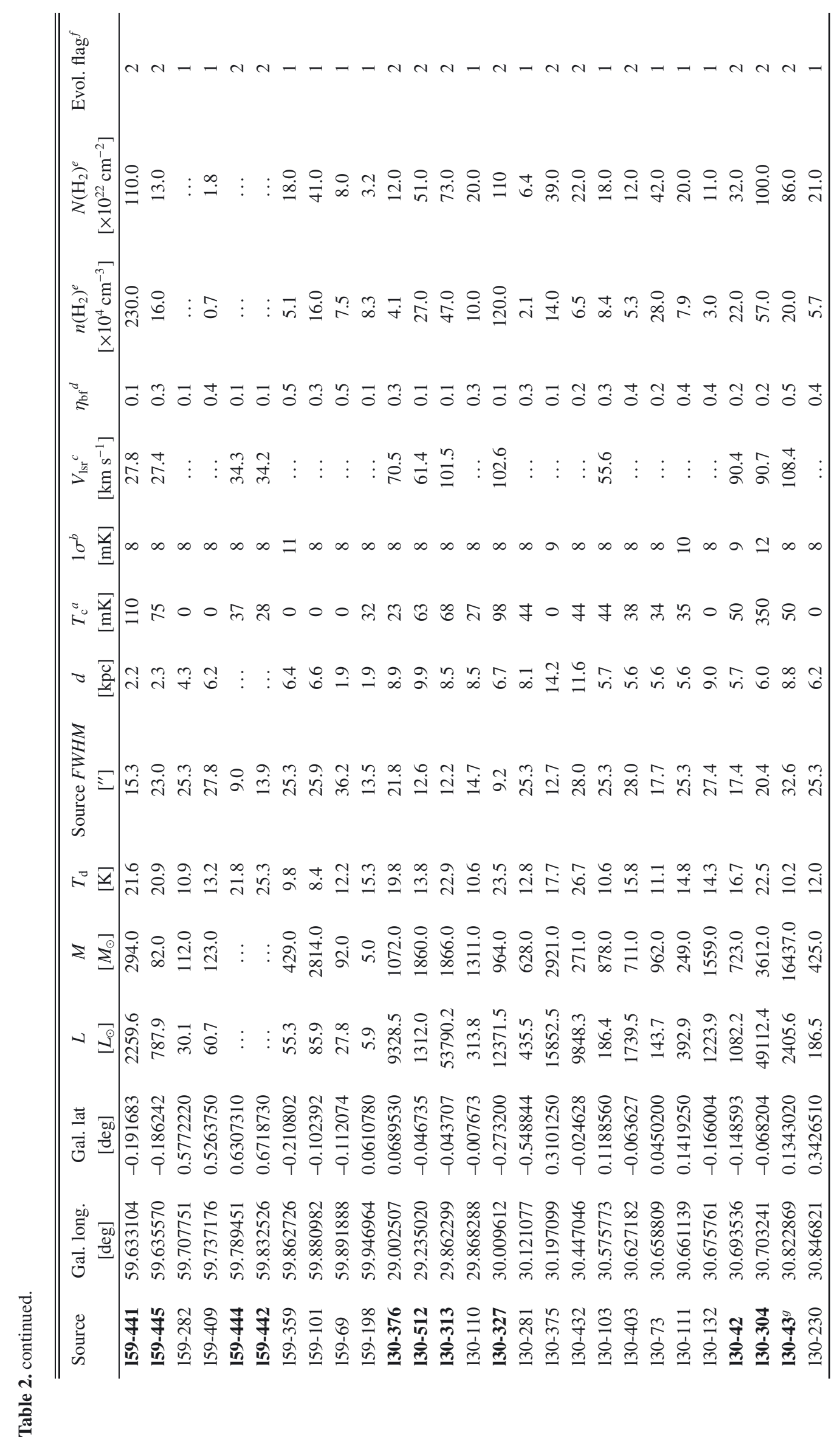


observing mode. The reference beams in these observations were located within $3^{\prime}$ on either side of the source. The observations were performed during the Herschel open time (OT2) on April 19 and 20, 2013, and the corresponding observation identifier (OBSID) range is [1342270449, 1342270604].

The data were processed using the standard Herschel interactive processing environment ${ }^{5}$, version 11.1, up to level 2, providing fully calibrated double side band spectra in the $T_{\mathrm{A}}^{*}$ antenna temperature intensity scale, in which the lines are calibrated on a single side band (SSB) scale. Because HIFI is intrinsically a double side band instrument, the continuum has to be divided by two to be properly scaled. The data quality is excellent with very low intensity ripples in most cases, typically below a few percent of the double side band continuum. However, in some cases the baselines are bad enough that the large rms $(\gtrsim 20-30 \mathrm{mK})$ does not enable a reliable estimate of the continuum temperature to be obtained (see Sect. 3.3). Therefore, for these sources we set the continuum temperature to 0. The FITS files were exported to the spectral line analysis software packages $\mathrm{xs}^{6}$ and $\mathrm{CLASS}^{7}$. Both polarizations and all three LO-settings were included in the averaged noise-weighted spectra. The resulting averages were convolved to a resolution of $1 \mathrm{~km} \mathrm{~s}^{-1}$ in sources with a low signal-to-noise ratio. All HIFI spectra, in $T_{\mathrm{A}}^{*}$ units, are shown in Appendices $\mathrm{A}\left(\ell=30^{\circ}\right.$ region $)$ and $\mathrm{B}\left(\ell=59^{\circ}\right.$ region $)$.

\section{Results}

\subsection{Distance determination}

Assigning a distance to sources detected with a photometer is a crucial step in giving physical significance to all information extracted from the Hi-GAL data. While reliable distance estimates are available for a limited number of known objects (e.g., HII regions, see Russeil 2003; and masers, see e.g., Green \& McClure-Griffiths 2011), this information does not exist for the majority of Hi-GAL sources. We therefore adopted the scheme presented by Russeil et al. (2011) to assign kinematic distances to large lists of sources. A ${ }^{12} \mathrm{CO}$ (or ${ }^{13} \mathrm{CO}$ ) spectrum (e.g., from the BU-FCRAO Galactic Ring Survey or GRS; Jackson et al. 2006) is extracted in the line of sight of every Hi-GAL source. Then, the velocity, $V_{\mathrm{lsr}}$, of the brightest spectral component is assigned to that specific source thereby allowing calculation of a kinematical distance. By using extinction maps (derived from the 2MASS point source catalog, see, e.g., Schneider et al. 2011) and a catalog of sources with known distances, such as HII regions, masers, and others, the distance ambiguity is resolved and a recommendation given. With this approach, it is possible to produce a distance map with the same pixel size of the $\mathrm{CO}$ cube as used to extract the spectra for every target, where the value of the pixel is the assigned distance of the Hi-GAL source(s) falling in that pixel. The typical error on the kinematic distance ${ }^{8}$ (Col. 8 in Table 2 ) is estimated to vary between $\sim 0.6-0.9 \mathrm{kpc}$ in the range of longitude $\ell \sim 30^{\circ}-60^{\circ}$.

\subsection{Profile decomposition}

The observed line profiles, and in particular those of the water spectral lines, show complex shapes, and likely represent

\footnotetext{
5 http://herschel.esac. esa.int/HIPE_download.shtml

6 http://www. chalmers.se/rss/oso-en/observations/ data-reduction-software

7 CLASS is part of the GILDAS software package developed by IRAM.

8 This estimate assumes that the distance ambiguity has been correctly solved.
}
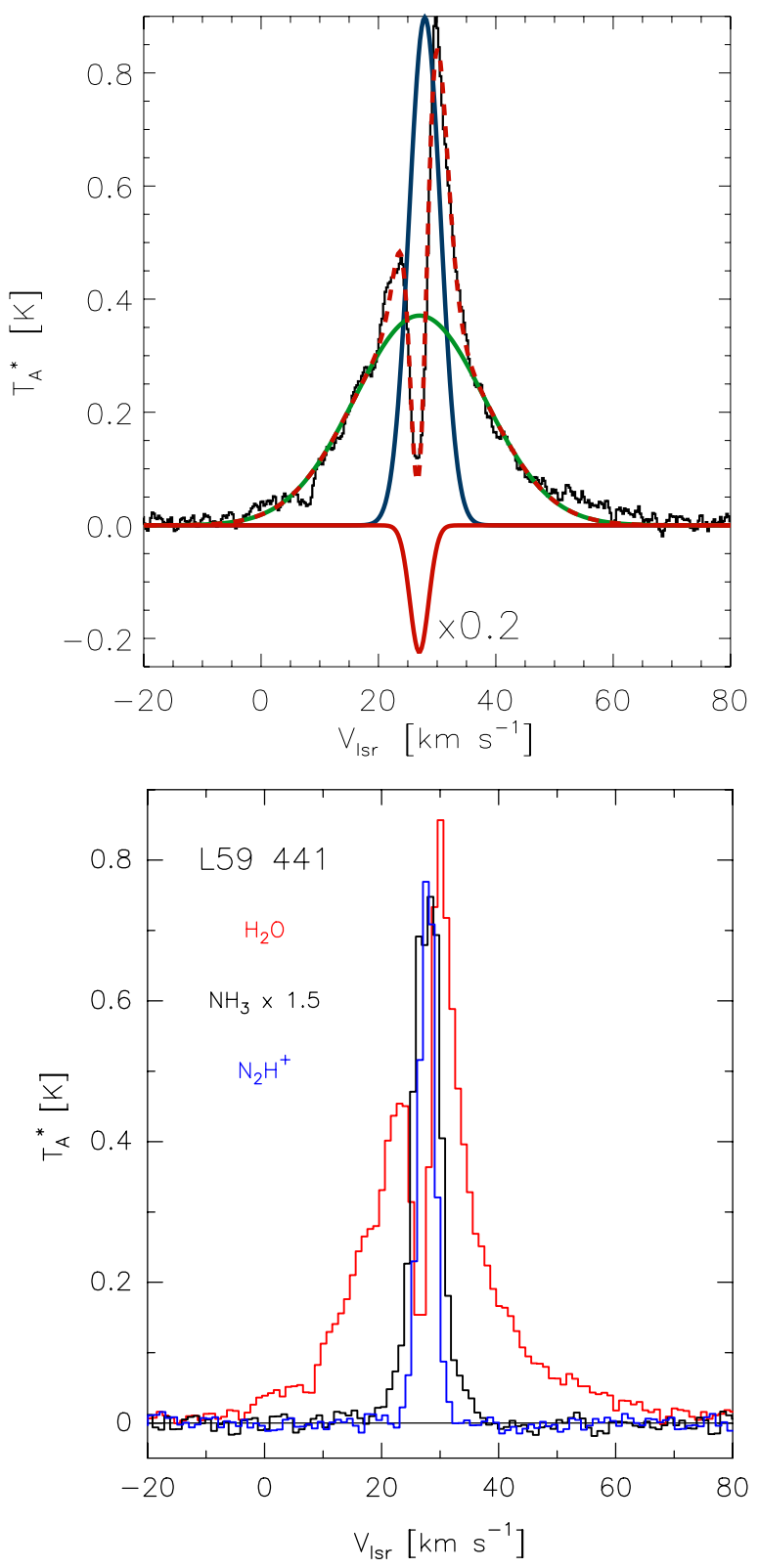

Fig. 1. Top panel: Gaussian decomposition of the $o-\mathrm{H}_{2} \mathrm{O}\left(1_{10}-1_{01}\right)$ spectrum toward the source 159-441. The individual Gaussian components are shown with solid color lines overlaid on the (continuumsubtracted) spectrum. The overall Gaussian fit is shown with a red dashed line, and the residual deviation at a velocity of $\simeq 55 \mathrm{~km} \mathrm{~s}^{-1}$ is visible. The intensity of the component in absorption has been multiplied by a 0.2 factor. Bottom panel: overlay of the $o-\mathrm{H}_{2} \mathrm{O}\left(1_{10}-1_{01}\right)$, $o-\mathrm{NH}_{3}\left(1_{0}-0_{0}\right)$ and $\mathrm{N}_{2} \mathrm{H}^{+}(6-5)$ lines toward the source $159-441$.

contributions from protostellar envelopes, molecular outflows, foreground clouds, as well as infall motions. These contributions, both in emission and in absorption, are disentangled and parameterized by fitting multiple Gaussians to the line profiles. We show an example in Fig. 1 and list all the results in Table 3. The Gaussian fits have been performed using up to four velocity components and all line parameters were allowed to vary. A similar decomposition of the $\mathrm{H}_{2} \mathrm{O}\left(1_{10}-1_{01}\right)(557 \mathrm{GHz})$ line has also been used by Kristensen et al. (2012) and Herpin et al. (2012).

Table 3 shows that the $\mathrm{H}_{2} \mathrm{O}$ line profiles of the $\ell=59^{\circ}$ sources are very similar. Almost all water spectra can be described as the sum of a narrow $\left(F W H M \sim 3-5 \mathrm{~km} \mathrm{~s}^{-1}\right)$, a medium $\left(F W H M \sim 6-10 \mathrm{~km} \mathrm{~s}^{-1}\right)$ and a broad (up to $\simeq 30 \mathrm{~km} \mathrm{~s}^{-1}$ ) 
Table 3. Gaussian fits of the Herschel-HIFI observations.

\begin{tabular}{|c|c|c|c|c|c|c|c|c|c|c|c|}
\hline Source & $\mathrm{H}_{2} \mathrm{O}$ Comp. ${ }^{a}$ & $\mathrm{E} / \mathrm{A}^{b}$ & $\begin{array}{c}\mathrm{H}_{2} \mathrm{O} \\
V_{\mathrm{lsr}} \\
{\left[\mathrm{km} \mathrm{s}^{-1}\right]}\end{array}$ & $\begin{array}{c}T_{\mathrm{A}}^{*} \\
{[\mathrm{~K}]}\end{array}$ & $\begin{array}{c}\Delta V \\
{\left[\mathrm{~km} \mathrm{~s}^{-1}\right]}\end{array}$ & $\begin{array}{c}V_{\mathrm{lsr}} \\
{\left[\mathrm{km} \mathrm{s}^{-1}\right]}\end{array}$ & $\begin{array}{c}\mathrm{N}_{2} \mathrm{H}^{+} \\
T_{\mathrm{A}}^{*} \\
{[\mathrm{~K}]} \\
\end{array}$ & $\begin{array}{c}\Delta V \\
{\left[\mathrm{~km} \mathrm{~s}^{-1}\right]}\end{array}$ & $\begin{array}{c}V_{\mathrm{lsr}} \\
{\left[\mathrm{km} \mathrm{s}^{-1}\right]}\end{array}$ & $\begin{array}{c}\mathrm{NH}_{3} \\
T_{\mathrm{A}}^{*} \\
{[\mathrm{~K}]} \\
\end{array}$ & $\begin{array}{c}\Delta V \\
{\left[\mathrm{~km} \mathrm{~s}^{-1}\right]}\end{array}$ \\
\hline \multirow[t]{3}{*}{$159-441$} & $\mathrm{M}$ & $\bar{E}$ & 27.9 & 0.89 & 6.3 & 27.8 & 0.83 & 3.1 & 27.7 & 0.81 & 3.6 \\
\hline & B & E & 27.0 & 0.37 & 26.0 & $\ldots$ & $\ldots$ & $\ldots$ & 28.2 & 0.09 & 10.1 \\
\hline & $\mathrm{N}$ & A & 26.9 & -1.1 & 3.7 & $\ldots$ & $\ldots$ & $\ldots$ & 27.7 & -0.46 & 2.0 \\
\hline \multirow[t]{3}{*}{$159-442$} & M & E & 36.7 & 0.24 & 6.3 & 34.2 & 0.14 & 3.6 & 34.8 & 0.13 & 6.4 \\
\hline & B & E & 36.7 & 0.12 & 23.6 & $\ldots$ & $\ldots$ & $\ldots$ & & $\ldots$ & \\
\hline & $\mathrm{N}$ & A & 34.9 & -0.30 & 4.8 & $\ldots$ & $\ldots$ & $\ldots$ & 35.1 & -0.05 & 2.5 \\
\hline \multirow[t]{4}{*}{$159-444$} & $\mathrm{~N}$ & E & 32.0 & 0.21 & 2.9 & 34.3 & 0.20 & 4.6 & 34.4 & 0.21 & 5.8 \\
\hline & B & $\mathrm{E}$ & 31.2 & 0.08 & 20.7 & $\ldots$ & $\ldots$ & $\ldots$ & $\ldots$ & $\ldots$ & $\ldots$ \\
\hline & $\mathrm{N}$ & A & 27.7 & -0.08 & 1.0 & $\ldots$ & $\ldots$ & $\ldots$ & $\ldots$ & $\ldots$ & $\ldots$ \\
\hline & $\mathrm{N}$ & A & 33.9 & -0.14 & 4.8 & $\ldots$ & $\ldots$ & $\ldots$ & 35.4 & -0.18 & 3.4 \\
\hline \multirow[t]{3}{*}{$159-445$} & M & E & 28.0 & 0.67 & 7.2 & 27.4 & 0.67 & 3.1 & 27.4 & 0.64 & 4.2 \\
\hline & B & E & 28.4 & 0.24 & 28.2 & $\ldots$ & $\ldots$ & $\ldots$ & $\ldots$ & . & \\
\hline & $\mathrm{N}$ & A & 27.0 & -0.80 & 3.7 & $\ldots$ & $\ldots$ & $\ldots$ & 27.7 & -0.25 & 1.7 \\
\hline $130-42$ & $\ldots$ & $\ldots$ & $\ldots$ & $\ldots$ & $\ldots$ & 90.4 & 0.03 & 1.9 & 91.3 & 0.08 & 4.2 \\
\hline $130-43^{d}$ & $\mathrm{~N}$ & E & 108.4 & 0.07 & 2.7 & $\ldots$ & $\lesssim 0.02^{c}$ & $\ldots$ & $\ldots$ & $\lessgtr 0.02^{c}$ & \\
\hline \multirow[t]{2}{*}{ 130-304 } & $\ldots$ & $\ldots$ & $\ldots$ & $\ldots$ & $\ldots$ & 90.7 & 0.86 & 4.1 & 87.8 & 0.33 & 3.1 \\
\hline & M & A & 92.9 & -0.35 & 8.5 & $\ldots$ & . & $\ldots$ & 93.7 & -0.31 & 5.7 \\
\hline $130-313$ & M & E & 101.2 & 0.07 & 6.5 & 101.5 & 0.08 & 2.7 & 101.5 & 0.14 & 5.8 \\
\hline \multirow[t]{2}{*}{$130-327$} & $\ldots$ & $\ldots$ & $\ldots$ & $\ldots$ & $\ldots$ & 102.6 & 0.07 & 3.6 & $\ldots$ & $\ldots$ & $\ldots$ \\
\hline & M & A & 103.8 & -0.11 & 5.7 & $\ldots$ & $\ldots$ & $\ldots$ & 104.6 & -0.08 & 4.6 \\
\hline $130-376$ & $\mathrm{~N}$ & E & 70.5 & 0.07 & 3.2 & $\ldots$ & $\lesssim 0.02^{c}$ & $\ldots$ & 70.5 & 0.05 & 2.7 \\
\hline \multirow[t]{3}{*}{$130-512$} & M & E & 63.2 & 0.11 & 5.5 & 61.4 & 0.06 & 2.9 & 61.8 & 0.15 & 2.5 \\
\hline & $\mathrm{N}$ & A & 59.5 & -0.06 & 2.4 & $\ldots$ & $\ldots$ & $\ldots$ & $\ldots$ & $\ldots$ & $\ldots$ \\
\hline & $\mathrm{N}$ & A & 64.0 & -0.06 & 1.6 & $\ldots$ & $\ldots$ & $\ldots$ & $\ldots$ & $\ldots$ & $\ldots$ \\
\hline
\end{tabular}

Notes. ${ }^{(a)}$ Definition of the Gaussian components of the water line: $\mathrm{N}=$ narrow, $\mathrm{M}=$ medium, $\mathrm{B}=$ broad. ${ }^{(b)} \mathrm{E}=$ emission, $\mathrm{A}=$ absorption. (c) $3 \sigma$ limit. ${ }^{(d)}$ Tentative detection (see Sect. 3.2).

velocity component. The narrow component is mostly seen in absorption, whereas the medium and broad components have positive intensities. Source $159-444$ shows an inverse PCygni profile overlaid on a broad component. In source 159-441 and, to a lesser extent, in 159-445 (these two sources are only $21.5^{\prime \prime}$ apart) if three Gaussian components are used in the fit, the redshifted wing of the spectrum still shows a residual deviation ( $23 \sigma$, see Fig. 1) from the overall fit at a velocity of $\simeq 55 \mathrm{~km} \mathrm{~s}^{-1}$. This residual can be eliminated with an additional Gaussian component, which may either have a high velocity $\left(\gtrsim 50 \mathrm{~km} \mathrm{~s}^{-1}\right.$ ), or a velocity comparable to the other components but with a wide line width $\left(\gtrsim 40 \mathrm{~km} \mathrm{~s}^{-1}\right)$. In the first case, the additional velocity component could be interpreted as an extremely high velocity (EHV) component. Similar EHV components have previously been reported, e.g., by Kristensen et al. (2012) toward several low-mass protostars and by Leurini et al. (2014) toward the massive protostar IRAS 17233-3606, and are usually associated with shocked gas. However, we do not have further evidence (such as spectra of other water lines and/or spectra at different positions) to confirm that an EHV component indeed exists in source 159-441. In the second scenario, an additional broad velocity component must be invoked, which may be caused by the fact that molecular outflows are not Guassian shaped. Because of this ambiguity, we choose to show only three Gaussian components for this source. Section 4.2 discusses the water line profile components in detail.

The situation is very different in the $\ell=30^{\circ}$ region, since no two profiles are identical. We have no clear detection of a broad component in the water spectra, and the line profile can be fit by fewer Gaussian components. Ammonia also displays complex line shapes with mixed emission and absorption, and only weak signs of outflows. In contrast to water and ammonia, the $\mathrm{N}_{2} \mathrm{H}^{+}$spectra do not show any line asymmetries and we observe only emission. In addition, two sources also show a regular or inverse P-Cygni profile, either in the water line (130-512) or in the $\mathrm{NH}_{3}$ spectrum (130-327) (see Sect. 4.2.5). Tables 2-4 mark source 130-43 as a tentative detection because recent (June 2015) follow-up observations with the 20-m telescope of the Onsala Space Observatory detected the $\mathrm{NH}_{3}(1,1)$ line toward this source at a velocity of $\simeq 95.3 \mathrm{~km} \mathrm{~s}^{-1}$, instead of $108.4 \mathrm{~km} \mathrm{~s}^{-1}$ as shown in Table 3 and Appendix A. Therefore, either the water line in source 130-43 is an artefact of some type, or both lines are actually real and thus the issue remains as to whether or not they belong to the same object. With our current data we cannot answer this question and therefore we assume here that the water line detection is real.

\subsection{Correlations between dust- and gas-derived physical parameters}

To better understand the nature of the detected sources in the $\ell=30^{\circ}$ and $\ell=59^{\circ}$ regions, as well as to determine the origin of the $\mathrm{H}_{2} \mathrm{O}$ emission, we performed correlations between several parameters. We begin with a comparison, which includes both dust-derived physical parameters, as determined from our previous Hi-GAL observations, and HIFI-derived parameters, such as the continuum temperature, $T_{\mathrm{c}}$. Then, the total $\mathrm{H}_{2} \mathrm{O}$ integrated line intensity is compared to different parameters.

In Fig. 2 we plot the clump luminosity vs. mass, as derived from our previous Hi-GAL observations. Since both are distance-dependent quantities, we only show sources where a distance could be determined. The plot shows that the clumps 
Table 4. Column densities and abundances from RADEX modeling.

\begin{tabular}{|c|c|c|c|c|c|c|c|c|c|c|}
\hline Source & Comp. ${ }^{a}$ & $\begin{array}{c}V_{\mathrm{lsr}} \\
{\left[\mathrm{km} \mathrm{s}^{-1}\right]}\end{array}$ & $\begin{array}{c}N\left(\mathrm{o}-\mathrm{H}_{2} \mathrm{O}\right)^{b} \\
{\left[\times 10^{14} \mathrm{~cm}^{-2}\right]}\end{array}$ & $\begin{array}{c}X\left(\mathrm{o}-\mathrm{H}_{2} \mathrm{O}\right)^{c} \\
{\left[\times 10^{-9}\right]}\end{array}$ & $\begin{array}{c}V_{\mathrm{lsr}} \\
{\left[\mathrm{km} \mathrm{s}^{-1}\right]}\end{array}$ & $\begin{array}{c}N\left(\mathrm{~N}_{2} \mathrm{H}^{+}\right)^{b} \\
{\left[\times 10^{14} \mathrm{~cm}^{-2}\right]}\end{array}$ & $\begin{array}{c}X\left(\mathrm{~N}_{2} \mathrm{H}^{+}\right)^{c} \\
{\left[\times 10^{-9}\right]}\end{array}$ & $\begin{array}{c}V_{\mathrm{lsr}} \\
{\left[\mathrm{km} \mathrm{s}^{-1}\right]}\end{array}$ & $\begin{array}{c}N\left(\mathrm{o}-\mathrm{NH}_{3}\right)^{b} \\
{\left[\times 10^{14} \mathrm{~cm}^{-2}\right]}\end{array}$ & $\begin{array}{c}X\left(\mathrm{o}-\mathrm{NH}_{3}\right)^{c} \\
{\left[\times 10^{-9}\right]}\end{array}$ \\
\hline \multirow[t]{3}{*}{$159-441$} & $\mathrm{M}$ & 27.9 & 450 & 41 & 27.8 & 0.34 & 6.2 & 27.7 & 37 & 3.4 \\
\hline & B & 27.0 & 67 & $\ldots$ & $\ldots$ & $\ldots$ & $\ldots$ & 28.2 & 1.3 & $\ldots$ \\
\hline & $\mathrm{N}$ & 26.9 & 0.34 & 0.06 & $\ldots$ & $\ldots$ & $\ldots$ & 27.7 & 0.05 & 0.01 \\
\hline \multirow{3}{*}{$159-442$} & M & 36.7 & $\ldots$ & $\ldots$ & 34.2 & $\ldots$ & $\ldots$ & 34.8 & $\ldots$ & $\ldots$ \\
\hline & B & 36.7 & $\ldots$ & $\ldots$ & $\ldots$ & $\ldots$ & $\ldots$ & $\ldots$ & $\ldots$ & $\ldots$ \\
\hline & $\mathrm{N}$ & 34.9 & $\ldots$ & $\ldots$ & $\ldots$ & $\ldots$ & $\ldots$ & 35.1 & $\ldots$ & $\ldots$ \\
\hline \multirow[t]{4}{*}{$159-444$} & $\mathrm{~N}$ & 32.0 & $\ldots$ & $\ldots$ & 34.3 & $\ldots$ & $\ldots$ & 34.4 & $\ldots$ & $\ldots$ \\
\hline & B & 31.2 & $\ldots$ & $\ldots$ & $\ldots$ & $\ldots$ & $\ldots$ & $\ldots$ & $\ldots$ & $\ldots$ \\
\hline & $\mathrm{N}$ & 27.7 & $\ldots$ & $\ldots$ & $\ldots$ & $\ldots$ & $\ldots$ & $\ldots$ & $\ldots$ & $\ldots$ \\
\hline & $\mathrm{N}$ & 33.9 & $\ldots$ & $\ldots$ & $\ldots$ & $\ldots$ & $\ldots$ & 35.4 & $\ldots$ & $\ldots$ \\
\hline \multirow[t]{3}{*}{$159-445$} & M & 28.0 & 350 & 270 & 27.4 & 32 & 25 & 27.4 & 60 & 46 \\
\hline & B & 28.4 & 300 & $\ldots$ & $\ldots$ & $\ldots$ & $\ldots$ & $\ldots$ & $\ldots$ & $\ldots$ \\
\hline & $\mathrm{N}$ & 27.0 & 0.36 & 0.6 & $\ldots$ & $\ldots$ & $\ldots$ & 27.7 & 0.03 & 0.04 \\
\hline $130-42$ & $\ldots$ & $\ldots$ & $\ldots$ & $\ldots$ & 90.4 & 4.3 & 1.3 & 91.3 & 6.0 & 1.9 \\
\hline $130-43^{g}$ & $\mathrm{~N}$ & 108.4 & 5 & 0.58 & & $\$ 30$ & $\$ 4$ & & $\lessgtr 0.6^{d}$ & $\lesssim 0.06^{d}$ \\
\hline \multirow[t]{2}{*}{ 130-304 } & & & & & 90.7 & 33 & 3.3 & 87.8 & 4.9 & 0.49 \\
\hline & M & 92.9 & $\gtrsim 2^{e}$ & $\gtrsim 0.4^{e}$ & $\ldots$ & $\ldots$ & $\ldots$ & 93.7 & 0.52 & 0.1 \\
\hline $130-313$ & M & 101.2 & 15 & 2.19 & 101.5 & 3.1 & 0.42 & 101.5 & 10 & 1.4 \\
\hline \multirow[t]{2}{*}{$130-327$} & .. & 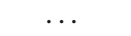 & .. & $\ldots$ & 102.6 & 2.4 & 0.22 & & .. & \\
\hline & M & 103.8 & 0.73 & 0.1 & $\ldots$ & $\ldots$ & $\ldots$ & 104.6 & 0.21 & 0.04 \\
\hline $130-376$ & $\mathrm{~N}$ & 70.5 & 39 & 33 & .. & $\lesssim 8$ & $\lesssim 7$ & 70.5 & 6.1 & 5.1 \\
\hline \multirow[t]{3}{*}{$130-512$} & M & 63.2 & 110 & 22 & 61.4 & 26 & 5.1 & 61.8 & 30 & 5.9 \\
\hline & $\mathrm{N}$ & 59.5 & 0.3 & 0.1 & $\ldots$ & $\ldots$ & $\ldots$ & $\ldots$ & $\ldots$ & $\ldots$ \\
\hline & $\mathrm{N}$ & 64.0 & 0.2 & 0.08 & $\ldots$ & $\ldots$ & $\ldots$ & $\ldots$ & $\ldots$ & $\ldots$ \\
\hline
\end{tabular}

Notes. Using our derived $n\left(\mathrm{H}_{2}\right)$ and $T_{\mathrm{k}}=T_{\text {dust }}$ (Table 2). ${ }^{(a)} \mathrm{N}=$ narrow component, $\mathrm{M}=$ medium, $\mathrm{B}=$ broad. ${ }^{(b)}$ Column density corrected for main beam efficiency and beam-filling factor (Table 2). ${ }^{\left({ }^{c}\right)}$ Abundance with respect to molecular hydrogen (Table 2). In the absorption components we used $N\left(\mathrm{H}_{2}\right) / 2$, assuming that the absorption is probing approximately half of the total column density in front of the background continuum. (d) $3 \sigma$ limit. ${ }^{(e)}$ Optically thick; $\tau$ is assumed to be 5. ${ }^{(f)}$ Tentative detection (see Sect. 3.2).

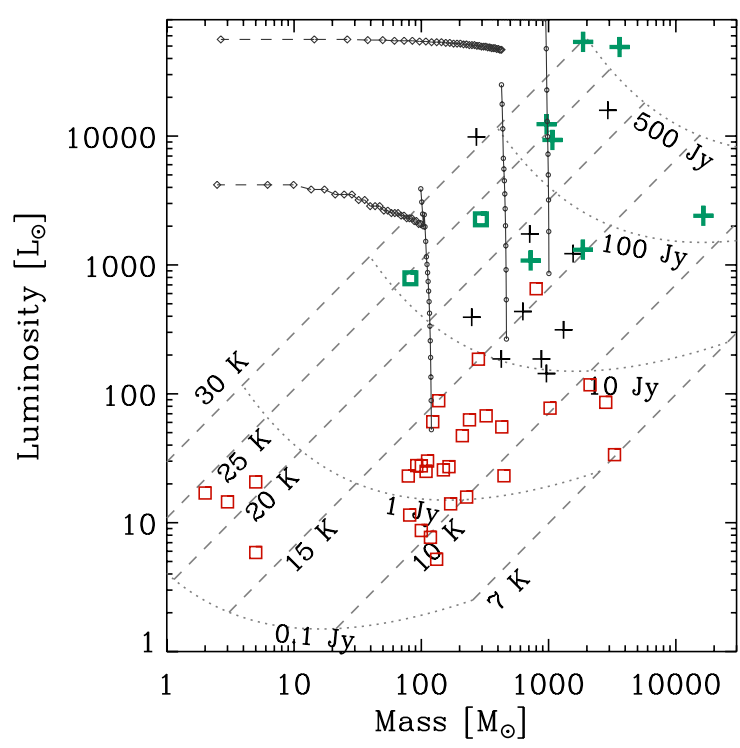

Fig. 2. Clump bolometric luminosity vs. mass, as derived from the HiGAL observations for all sources where a distance could be determined. Sources in the $\ell=30^{\circ}$ and $\ell=59^{\circ}$ regions are represented by the (black) "+" sign and (red) open squares, respectively. Sources with a detection in at least one of the three observed spectral lines are shown with thicker (green) symbols. The dashed lines are loci at constant temperature. Roughly orthogonal to these are loci (dotted lines) of constant $250 \mu \mathrm{m}$ flux density, ranging from 0.1 to $500 \mathrm{Jy}$, assuming a modified blackbody spectral energy distribution with $\beta=1.5$ and a fixed distance of $\sim 6 \mathrm{kpc}$, equal to the median of the distances of the observed sources. The black dots and the lines joining them represent the evolutionary tracks of clumps with starting envelope mass equal to 120,470 and $10^{3} M_{\odot}$ (see Sect. 4.4). found in the $\ell=59^{\circ}$ region are characterized by lower luminosity and mass compared to those in the $\ell=30^{\circ}$ region (see the detailed statistical analysis by Olmi et al. 2013 and Olmi et al. 2014a). Sources with a detection in at least one of the three observed spectral lines are clearly found at higher luminosity $\left(L \gtrsim 10^{3} L_{\odot}\right)$, as also confirmed by Table 5 . In Fig. 2 we also plot a series of evolutionary tracks, which we discuss in Sect. 4.4.

In Fig. 3 we plot the dust-derived temperatures $\left(T_{\mathrm{d}}\right.$, Col. 6 in Table 2) and luminosities of the clumps in the $\ell=30^{\circ}$ and $\ell=59^{\circ}$ regions vs. $T_{\mathrm{c}}$. The continuum flux density associated with $T_{\mathrm{c}}$ is likely to have a contribution from both free-free emission and warm dust thermal emission. We do not have an alternative method to determine the contribution of free-free emission in our sources. Furthermore, because we have only measured $T_{\mathrm{c}}$ at one frequency, we cannot determine how $T_{\mathrm{c}}$ varies with frequency, which could otherwise be used to discriminate between dust and free-free emission. van der Tak et al. (2013), however, found that the free-free emission in their massive protostellar clumps is negligible compared to warm dust emission. If this is also true for our sources, then $T_{\mathrm{c}}$ should correlate with the source bolometric luminosity. This is indeed shown in the top panel of Fig. 3, where we also used the Bayesian IDL routine LINMIX_ERR to perform a linear regression to find the slope of the best-fit line. The source luminosity is expected to increase with time, as the clump evolves from the starless to the protostellar phase (see, e.g., Molinari et al. 2008). Therefore, the top panel of Fig. 3 is also likely to represent an evolutionary sequence, however, the bottom panel of Fig. 3 shows that there is only a marginal correlation between $T_{\mathrm{d}}$ and $T_{\mathrm{c}}$, which are both distance independent. The large scatter is certainly in part due to 
Table 5. Mean values of bolometric luminosity, mass, and continuum temperature.

\begin{tabular}{lcccccrr}
\hline \hline \multirow{2}{*}{ Region } & \multicolumn{3}{c}{ Sources with detection } & & \multicolumn{3}{c}{ Sources with no detection ${ }^{a}$} \\
\cline { 2 - 3 } & Mean $L$ & Mean $M$ & Mean $T_{\mathrm{c}}$ & & Mean $L$ & Mean $M$ & Mean $T_{\mathrm{c}}$ \\
& {$\left[L_{\odot}\right]$} & {$\left[M_{\odot}\right]$} & {$[\mathrm{K}]$} & & {$\left[L_{\odot}\right]$} & {$\left[M_{\odot}\right]$} & {$[\mathrm{K}]$} \\
\hline$\ell=30$ & $16200 \pm 17600$ & $3430 \pm 3300$ & $93 \pm 65$ & & $2145 \pm 2570$ & $690 \pm 305$ & $37 \pm 5$ \\
$\ell=59$ & $1520 \pm 735$ & $190 \pm 105$ & $63 \pm 30$ & & $90 \pm 64$ & $440 \pm 390$ & $26 \pm 4$ \\
\hline
\end{tabular}

Notes. ${ }^{(a)}$ Only sources where all three parameters could be measured were included.
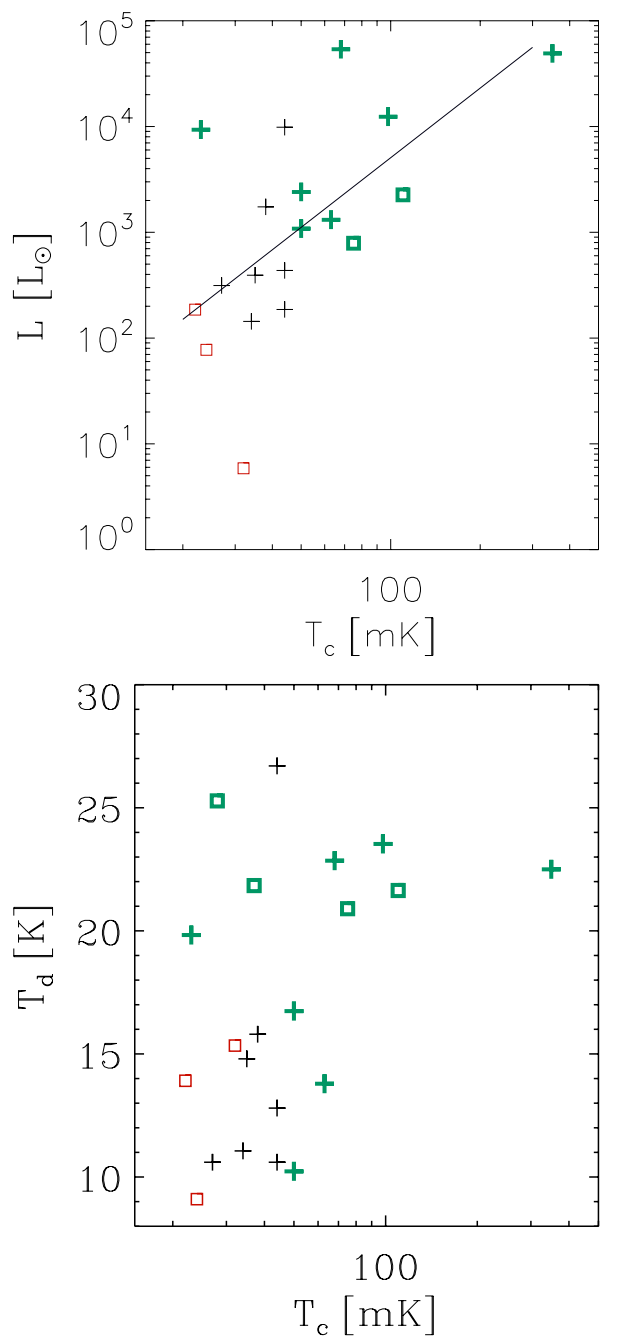

Fig. 3. Top panel: clump bolometric luminosity vs. continuum temperature, $T_{\mathrm{c}}$. The solid line represents the linear fit to all points (see text); the Spearman rank coefficient is 0.65. Bottom panel: dust-derived temperature, $T_{\mathrm{d}}$, vs. continuum temperature. Symbols are as in Fig. 2.

the large uncertainties in $T_{\mathrm{c}}$, since the baseline root mean square (rms) was in general large ( $\gtrsim 20-30 \mathrm{mK})$.

In Fig. 4 we plot various line integrated intensities. The integrated area of the profile has been determined by summing all components in emission. For a Gaussian line profile, the integrated emission is $T_{\mathrm{A}}^{\mathrm{pk}} \Delta V \sqrt{\pi /(4 \ln 2)}$, where $T_{\mathrm{A}}^{\mathrm{pk}}$ is the peak antenna temperature and $\Delta V$ is the FWHM of the line. We did not consider sources with pure absorption spectra in any of the plotted spectral lines. Despite the low number of data points, the top and middle panels show an excellent correlation between the integrated intensities of the $\mathrm{H}_{2} \mathrm{O}$ and $\mathrm{NH}_{3}$ lines, and also between those of the two N-bearing molecules. In the middle panel, we did not include source 130-304 in the plot because it has a completely self-absorbed water line profile, and the $\mathrm{NH}_{3}$ line has an inverse P-Cygni profile. Therefore, the integrated intensity (in emission) of ammonia is very uncertain. The reasonable agreement between the integrated intensities of ammonia and $\mathrm{N}_{2} \mathrm{H}^{+}$suggests that they are emitted from similar volumes of gas, assuming similar excitation conditions.

Finally, in the bottom panel of Fig. 4 we plot the quantity $\int T_{\mathrm{A}}^{*}\left[\mathrm{H}_{2} \mathrm{O}\right] \mathrm{d} v \times d^{2}$, proportional to the $\mathrm{H}_{2} \mathrm{O}$ line luminosity, vs. the continuum temperature, $T_{\mathrm{c}}$. Although we do not show any linear fit in this case, there seems to be a general trend of increasing line luminosity with $T_{\mathrm{c}}$. The 130 source with the highest water line luminosity ( $~ Z 60 \mathrm{~K} \mathrm{~km} \mathrm{~s}^{-1} \mathrm{kpc}^{2}$ ) is 130-512, which has a P-Cygni profile (see Sect. 4.2.5) and thus the actual line luminosity is uncertain. Except for this point, the plot shows that in these objects the typical water line luminosity toward the $\ell=30^{\circ}$ sources is lower than that of the $\ell=59^{\circ}$ region.

\subsection{Column densities and molecular abundances}

\subsubsection{RADEX modeling}

Since we lack direct information about the excitation temperatures and opacities of the observed transitions, we use the nonequilibrium homogeneous radiative transfer code RADEX ${ }^{9}$ (van der Tak et al. 2007) to correct for possible populations of molecules in unobserved excited levels to relate the observed emission and absorption components to the column densities of $\mathrm{H}_{2} \mathrm{O}, \mathrm{NH}_{3}$, and $\mathrm{N}_{2} \mathrm{H}^{+}$.

The principal parameters needed to specify a model are the kinetic temperature, $T_{\mathrm{k}}$, the average number density of molecular hydrogen $n_{\mathrm{H} 2}$, and the line width (see, e.g., Persson et al. 2009). The density of each source is computed from the Hi-GAL observed size and mass, assuming a homogeneous, spherical cloud. The kinetic temperature is assumed to be equal to the dust temperature obtained from our Hi-GAL observations. All parameters are listed in Table 2. The background radiation field is an average of the Galactic background radiation in the solar neighborhood and of the cosmic microwave background radiation.

The column density obtained from RADEX is varied until the model line brightness temperature, $T_{\mathrm{b}}$, matches the amplitude of the Gaussian fit (Table 3 ). The line brighness temperature is obtained from the antenna temperature after correcting for mainbeam efficiency, $\eta_{\mathrm{mb}}$, and beam-filling factor, $\eta_{\mathrm{bf}}$, i.e.,

$T_{\mathrm{A}}^{*}=T_{\mathrm{b}} \eta_{\mathrm{mb}} \eta_{\mathrm{bf}}=T_{\mathrm{b}} \eta_{\mathrm{mb}} \frac{\theta_{\mathrm{mb}}^{2}}{\theta_{\mathrm{mb}}^{2}+\theta_{\mathrm{s}}^{2}}$,

where $\theta_{\mathrm{mb}}$ and $\theta_{\mathrm{s}}$ are the FWHM of the main beam and source, respectively. The values of $\eta_{\mathrm{bf}}$ are also listed in Table 2 . For the

9 http://www.sron.rug.nl/ vdtak/radex/radex.php 

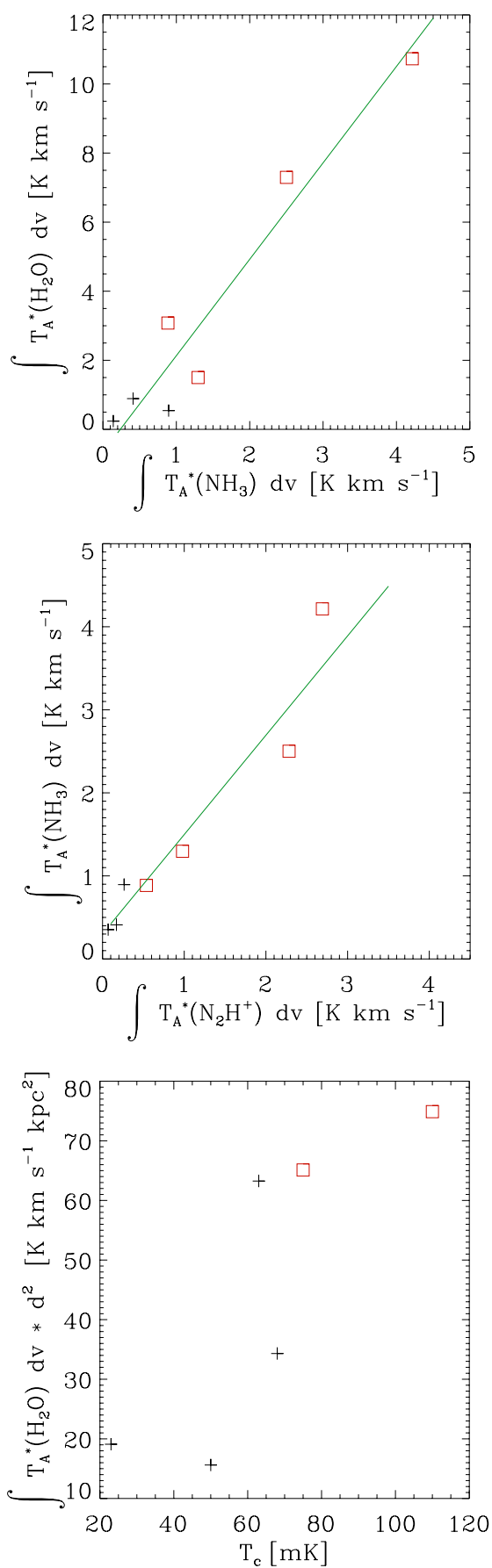

Fig. 4. Top panel: integrated $\mathrm{H}_{2} \mathrm{O}\left(1_{10}-1_{01}\right)$ line intensity plotted vs. the integrated $\mathrm{NH}_{3}\left(1_{0}-0_{0}\right)$ line intensity. Symbols are as in Fig. 2. The solid line represents the linear fit to all points. The Spearman rank coefficient is 0.94 . Middle panel: integrated $\mathrm{NH}_{3}\left(1_{0}-0_{0}\right)$ line intensity vs. the integrated $\mathrm{N}_{2} \mathrm{H}^{+}(6-5)$ line intensity. Source 130-304 has not been included in the plot (see text). The Spearman rank coefficient is 0.96 . Bottom panel: $\int T_{\mathrm{A}}^{*}\left[\mathrm{H}_{2} \mathrm{O}\right] \mathrm{d} v \times d^{2}$, proportional to the $\mathrm{H}_{2} \mathrm{O}$ line luminosity, vs. continuum temperature, $T_{\mathrm{c}}$. Symbols are as in Fig. 2.

components in absorption, we match the observed and modeled integrated opacity instead of the brightness temperature (see Sect. 4.2.3).

The computed RADEX results are listed in Table 4 . The results are not very sensitive to changes in density because of the high critical densities $\left(n_{\mathrm{cr}} \gtrsim 10^{7}-10^{8} \mathrm{~cm}^{-3}\right)$ of all three molecular transitions. However, the column density is sensitive to changes in kinetic temperature if $T_{\mathrm{k}} \lesssim 15 \mathrm{~K}$. For sources with nondetections in one (or two) of the molecular transitions, we estimated the upper limit to the column density with $3 \sigma$ limits for $T_{\mathrm{A}}^{*}$ listed in Table 3.

Abundances with respect to molecular hydrogen, $X(\mathrm{~mol})=$ $N(\mathrm{~mol}) / N\left(\mathrm{H}_{2}\right)$, are estimated using the derived volume densities and observed source sizes to compute $N\left(\mathrm{H}_{2}\right)$. For the components in absorption, we divide the estimated $N\left(\mathrm{H}_{2}\right)$ by a factor of two since the absorption is probing half of the total column density that is in front of the continuum source. For the outflow components, we cannot estimate the abundance since $N\left(\mathrm{H}_{2}\right)$ is likely orders of magnitude lower than that of the whole cloud.

Table 4 indicates that the water molecular abundance is possibly higher toward sources in the $\ell=59^{\circ}$ region. Our results may be affected by the uncertainties in the derived Hi-GAL physical parameters and other assumptions. However, combined with the result that the typical water line luminosity toward the $\ell=30^{\circ}$ sources (where no broad component is detected) is lower compared to that of the $\ell=59^{\circ}$ region (see bottom panel of Fig. 4), we conclude that our observations support a scenario where the water abundance is linked to shocked gas.

\subsubsection{Column density for the absorption component}

We also used an alternative approach to compute the $o-\mathrm{H}_{2} \mathrm{O}$ column density of the envelope gas responsible for the observed absorption in the water lines, which we also use to double check the RADEX results. We first derived the optical depth at the dip of the absorption profile as

$\tau_{\text {dip }}=-\ln \left(\frac{T_{\mathrm{A}}^{\mathrm{em}}-T_{\mathrm{A}}^{\mathrm{dip}}}{T_{\mathrm{A}}^{\mathrm{em}}}\right)$,

where, in order to include the line contribution to the absorption background, the antenna temperature $T_{\mathrm{A}}^{\mathrm{em}}$ represents the sum of all Gaussian components in emission (at the velocity of the dip in the absorption component) and of the continuum temperature $T_{\mathrm{c}}$. This procedure assumes that the absorber is located in front of both the continuum and the line emitting source. The parameter $T_{\mathrm{A}}^{\mathrm{dip}}$ represents the peak absolute value of the antenna temperature of the Gaussian component in absorption. The integrated optical depth of the absorption conponent is then calculated as $\tau_{\mathrm{abs}}=\int \tau \mathrm{d} v=1.06 \tau_{\text {dip }} \Delta V_{\mathrm{abs}}$, where $\Delta V_{\mathrm{abs}}$ represents the line FWHM of the Gaussian component in absorption. Finally, the column density can be estimated as

$N_{\mathrm{tot}} \simeq N_{l}=\frac{8 \pi v_{\mathrm{ul}}^{3} g_{l}}{c^{3} A_{\mathrm{ul}} g_{u}}\left[1-\exp \left(-\frac{h \nu_{\mathrm{ul}}}{k T_{\mathrm{ex}}}\right)\right]^{-1} \tau_{\mathrm{abs}}$,

where $A_{\mathrm{ul}}$ and $v_{\mathrm{ul}}$ represent the Einstein $A$ coefficient and the frequency associated with the transition between the lower and upper level, indicated by the subscripts $l$ and $u$, respectively, and with degeneracies $g_{l}$ and $g_{u}$. Physical constants are indicated with standard symbols and $T_{\mathrm{ex}}$ represents the excitation temperature. Since we do not know $T_{\text {ex }}$ we assume for the moment that $T_{\text {ex }} \ll h v_{\text {ul }} / k=26.6 \mathrm{~K}$ for the $557 \mathrm{GHz}$ water line. Therefore, the exp factor in Eq. (3) can be neglected.

We thus obtain remarkably similar $o-\mathrm{H}_{2} \mathrm{O}$ column densities, varying from about $10^{13} \mathrm{~cm}^{-2}$ to $\simeq 5 \times 10^{13} \mathrm{~cm}^{-2}$ (consistent with the RADEX results), with the highest value obtained toward the source 130-304, where in fact the absorption appears to be almost saturated since the line profile is nearly down to the zero-temperature level. The column densities of the outer 
envelope are thus quite similar to those estimated for both low(Kristensen et al. 2012) and high-mass (van der Tak et al. 2013), star-forming regions.

\section{Discussion}

\subsection{Average physical conditions in the $\ell=30^{\circ}$ and $\ell=59^{\circ}$ regions}

As shown by Tables 2 and 3 , in the $\ell=30^{\circ}$ region about $41 \%$ of the sources have a detection in at least one of the observed spectral lines. This percentage drops to about $11 \%$ in the $\ell=$ $59^{\circ}$ region. All of the sources with at least one detection are protostellar in both galactic regions. The significant difference in detections between the two regions may be a consequence of the different fraction of protostellar sources observed in each region, corresponding to $59 \%$ and $14 \%$ in $\ell=30^{\circ}$ and $\ell=59^{\circ}$, respectively. In fact, the fraction of protostellar sources with at least one detection corresponds to $70 \%$ and $80 \%$ in the $\ell=30^{\circ}$ and $\ell=59^{\circ}$ regions, respectively, making the difference in the detection rate less significant.

In Table 5 we list the mean values of the clump mass and bolometric luminosity, as derived from the Hi-GAL observations, for the observed sources in the two galactic regions. The mean values of the luminosity for sources with a detection in one of the observed spectral lines are much higher than the luminosity of sources with no detection at all. The scatter around the mean value is high and thus our conclusion is only tentative and needs more data to be confirmed. Furthermore, the mean values in the $\ell=59^{\circ}$ region are derived from only two sources, since in sources 159-442 and 159-444 the distance could not be determined reliably.

As with luminosity, Table 5 shows that $T_{\mathrm{c}}$ has a higher mean value (by more than a factor of 2) in sources with at least one detected spectral line for both observed regions. Given that all of these sources are protostellar, the mean values of $T_{\mathrm{c}}$ for sources with and without detection are in fact very similar to the mean values of $T_{\mathrm{c}}$ for protostellar and starless sources, respectively. Compared to luminosity and mass, the mean values of $T_{\mathrm{c}}$ show a lower scatter, which is still high only in the case of sources with detection in the $\ell=30^{\circ}$ region. In fact, the scatter for the case of sources with no detection is quite low in both regions, despite the low number of sources. Given the marginal correlation between luminosity and $T_{\mathrm{c}}$ (see Sect. 3.3) this result is not totally unexpected. However, given that the continuum temperature is a distance-independent quantity, this result is more significant in charaterizing the sources. Finally, Table 5 also reveals that the mean values of the mass for sources with and without a detection do not show any specific relation. Thus, if we tentatively assume that the difference in the mean value of luminosity (and $T_{\mathrm{c}}$ ) between sources with and without detections is indeed real, then this might be a consequence of evolutionary effects rather then an effect associated with more massive clumps (see also Sect. 4.4).

\subsection{Gas kinematics}

Our decomposition of the $\mathrm{H}_{2} \mathrm{O}$ line profiles (see Sect. 3.2) is similar to that of Kristensen et al. (2012) toward lowmass protostars, and to the description of the $\mathrm{H}_{2} \mathrm{O}$ profiles of van der Tak et al. (2013) toward high-mass protostars. Herpin et al. (2012) found a similar profile decomposition toward the massive protostar W43-MM1. In the following, first we describe the general properties of the line shapes in the $\ell=30^{\circ}$ and $\ell=59^{\circ}$ regions, and then we discuss the possible physical origin of each component.

\subsubsection{Line profile components in the $\ell=59^{\circ}$ region}

Here we summarize the main observational properties of the line profiles of the three transitions, $\mathrm{H}_{2} \mathrm{O}\left(1_{10}-1_{01}\right), \mathrm{NH}_{3}\left(1_{0}-0_{0}\right)$, and $\mathrm{N}_{2} \mathrm{H}^{+}(6-5)$ toward the $\ell=59^{\circ}$ region.

$\mathrm{H}_{2} \mathrm{O}$. All detected sources of the $\ell=59^{\circ}$ region have a strikingly similar water line profile, which is always charaterized by an absorption dip at the source velocity (as given by $\mathrm{N}_{2} \mathrm{H}^{+}$). This absorption dip does not appear to be saturated. As shown by Table 3, the offset between these absorption components and the source velocity does not appear to be significant. The broad and medium Gaussian components have line widths exceeding those of $\mathrm{N}_{2} \mathrm{H}^{+}$, which trace the more quiescent ambient gas, and are thus likely originating in protostellar outflows. The water line profile in source 159-444 is somewhat different from that of the other three detected sources in the $\ell=59^{\circ}$ region since it is charaterized by an inverse P-Cygni profile overlaid on a broad component (see Sect. 4.2.5).

$\mathrm{N}_{2} \mathrm{H}^{+}$and $\mathrm{NH}_{3}$. While the $\mathrm{N}_{2} \mathrm{H}^{+}$line-profile looks Gaussian and thus is used to set the source velocity, $\mathrm{NH}_{3}$ shows an asymmetric line profile, which exhibits self-absorption (less evident in 159-442), though not at the exact same velocity as absorption in the water line (see Table 3). Absorption is also present in the $\mathrm{NH}_{3}$ spectrum of 159-444. Finally, one of the Gaussian components of the $\mathrm{NH}_{3}$ line toward sources 159-441 and 159-442 has a line width of $10.1 \mathrm{~km} \mathrm{~s}^{-1}$ and $6.4 \mathrm{~km} \mathrm{~s}^{-1}$ (see Table 3). This suggests that the outflows observed in the water line are also partially detected in the ammonia line, at least in source 159-441.

\subsubsection{Line profile components in the $\ell=30^{\circ}$ region and comparison of line widths}

The nature of the observed lines toward this region is significantly different from that of the sources in the $\ell=59^{\circ}$ region. The line intensities are in general lower compared to those measured toward the $\ell=59^{\circ}$ region, which is likely a consequence of the average larger distance to the $\ell=30^{\circ}$ sources.

$\mathrm{H}_{2} \mathrm{O}$. The water line is detected both in emission and absorption, but the line profiles are much more varied. As already mentioned, in the sources 130-304 and 130-327 the Gaussian component in absorption appears to be almost saturated. No line wings are detected above the noise level. In several sources (e.g., 130-304 and 130-327), the presence of multiple water absorption lines is evidently tracing the diffuse interstellar gas (which will be the subject of a separate paper, Persson et al., in prep.), which are likely a consequence of the larger amount of diffuse gas encountered along the $\ell=30^{\circ}$ direction. In Fig. 4 we have already shown that the water line luminosity toward the $\ell=30^{\circ}$ sources is lower compared to that of the $\ell=59^{\circ}$ region.

$\mathrm{N}_{2} \mathrm{H}^{+}$and $\mathrm{NH}_{3}$. As for the $\ell=59^{\circ}$ region, the $\mathrm{N}_{2} \mathrm{H}^{+}$line profile looks Gaussian, and (when detected) it is also used to set the source velocity. In most cases the line profiles of water and ammonia are consistent, i.e., they are either both in emission or absorption. The $\mathrm{NH}_{3}$ spectrum exhibits an inverse P-Cygni profile toward source 130-304, and the component in absorption looks almost saturated as in the water line. Ammonia also shows absorption lines along the line of sight toward source 130-327 (and possibly 130-42), as already observed in the water spectra. The maximum velocity difference between $\mathrm{NH}_{3}$ and $\mathrm{N}_{2} \mathrm{H}^{+}$, when both lines are seen in emission, is $\simeq 0.9 \mathrm{~km} \mathrm{~s}^{-1}$ in source $130-42$. 
L. Olmi et al.: High-mass starless and protostellar clumps identified by the Hi-GAL survey
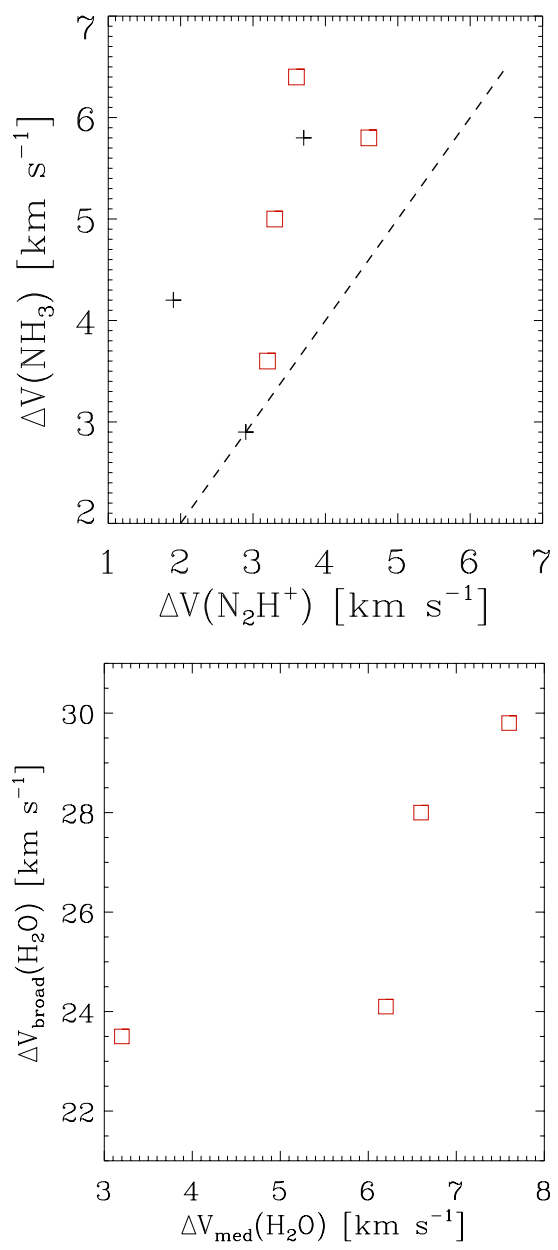

Fig. 5. Top: line width of the $\mathrm{NH}_{3}$ line vs. line width of the $\mathrm{N}_{2} \mathrm{H}^{+}$line, when both are detected in emission. The dashed line represents the $\Delta V\left(\mathrm{NH}_{3}\right)=\Delta V\left(\mathrm{~N}_{2} \mathrm{H}^{+}\right)$locus. Bottom: line width of the broad component of the water line profile vs. line width of the medium component for the $\ell=59^{\circ}$ sources. Symbols are as in Fig. 2.

We compared the line widths of the $\mathrm{NH}_{3}$ and $\mathrm{N}_{2} \mathrm{H}^{+}$lines, as shown in Fig. 5. In all of the sources except for source 130304, which shows an inverse P-Cygni profile in the ammonia spectrum, we find that $\Delta V\left(\mathrm{NH}_{3}\right) \geq \Delta V\left(\mathrm{~N}_{2} \mathrm{H}^{+}\right)$, including all four detected sources in the $\ell=59^{\circ}$ region. We also find that $\Delta V\left(\mathrm{NH}_{3}\right)$ tends to be somewhat higher in the $\ell=59^{\circ}$ sources. Since molecular outflows are the most distinctive feature of the detected $\ell=59^{\circ}$ sources, this is an indication that the line width of $\mathrm{NH}_{3}$ is being broadened by these outflows. Likewise, the $\mathrm{N}_{2} \mathrm{H}^{+}$emission must then originate in a volume where the molecular gas is not significantly affected by the molecular outflows.

\subsubsection{Narrow component}

The general appearance of the narrow component, mostly in absorption, indicates a high $\mathrm{H}_{2} \mathrm{O}$ column density and a low excitation temperature, suggesting that the absorption is being caused by the outer envelope and ambient cloud. Source 130-376 is the only source showing a clear narrow component in emission, which could be related to temperature, as appearance in emission requires an excitation temperature above the continuum level. In fact, 130-376 has the lowest continuum temperature $(23 \mathrm{mK}$, see Table 2$)$ among sources in the $\ell=30^{\circ}$ region with a detectable continuum emission. As mentioned earlier, in
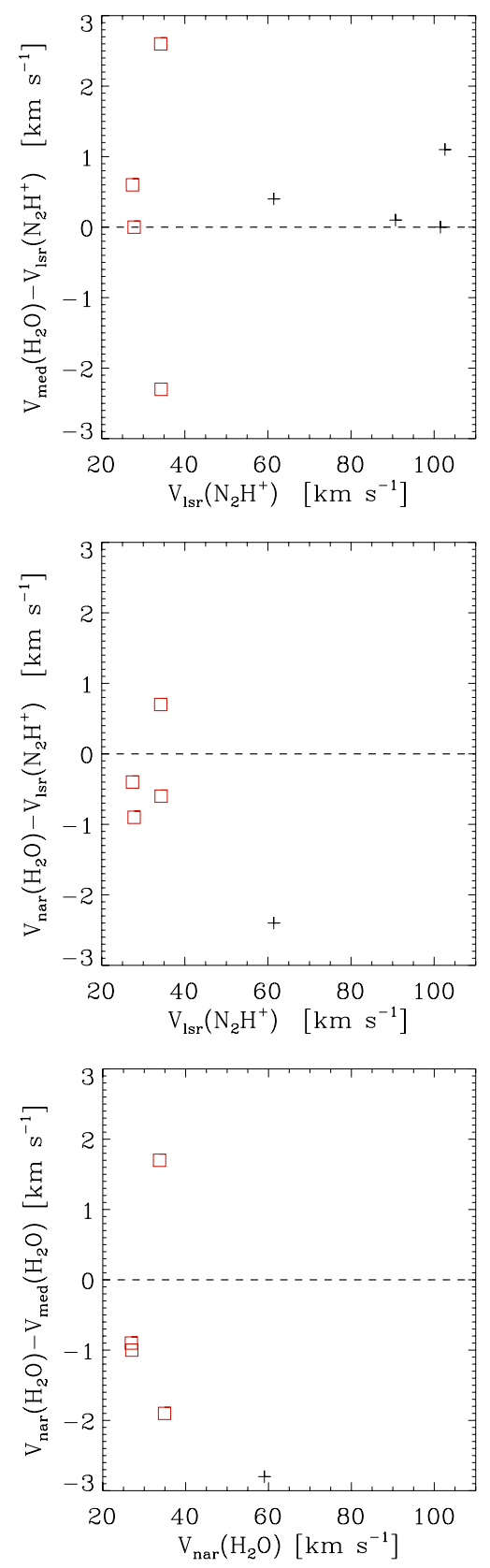

Fig. 6. Central velocities of the narrow $\left(V_{\text {nar }}\right)$ and medium $\left(V_{\text {med }}\right)$ Gaussian components of the observed $\mathrm{H}_{2} \mathrm{O}$ lines compared with each other and with the systemic velocity from $\mathrm{N}_{2} \mathrm{H}^{+}$. Symbols are as in Fig. 2.

the other $\ell=30^{\circ}$ sources the water line, either in absorption or emission, is broader than $\simeq 5 \mathrm{~km} \mathrm{~s}^{-1}$ and has thus been classified as a medium component.

Following Kristensen et al. (2012), since the absorption of the narrow component is seen against both the outflow and continuum emission, the absorbing layer must be located in front of both the emitting layers, i.e., the outflows must also be embedded. Figure 6 shows that the narrow component is either centered at the source velocity (offset $\lesssim 1 \mathrm{~km} \mathrm{~s}^{-1}$ ) or blueshifted (130-512) relative to the systemic velocity, as given by $\mathrm{N}_{2} \mathrm{H}^{+}$. In most $\ell=59^{\circ}$ sources, however, the narrow component is also slightly blushifted relative to $\mathrm{N}_{2} \mathrm{H}^{+}$emission, suggesting that the envelope, where the absorbed narrow component originates, is expanding. In addition, Fig. 6 shows that the narrow component is also blushifted relative to both the medium and broad 
(not shown) components. The only source where $V_{\text {nar }}\left(\mathrm{H}_{2} \mathrm{O}\right)$ is redshifted compared to both $V_{\text {med }}\left(\mathrm{H}_{2} \mathrm{O}\right)$ and $V_{\text {broad }}\left(\mathrm{H}_{2} \mathrm{O}\right)$ is 159-444, which has an inverse P-Cygni profile, typical of an infalling envelope.

Contrary to van der Tak et al. (2013) we do not find any correlation between the source mass (which is mostly due to the envelope contribution, and is derived from the Hi-GAL observations of cold dust emission) and the line width of the narrow component. In Sect. 3.4 using RADEX we estimated the $o-\mathrm{H}_{2} \mathrm{O}$ column density of the envelope gas responsible for the observed absorption toward all $\ell=59^{\circ}$ sources and toward the $\ell=30^{\circ}$ sources 130-304, 130-327 and 130-512 (see also Table 3).

\subsubsection{Outflows and shocked gas}

The $\mathrm{H}_{2} \mathrm{O}$ line profiles are characterized by two additional and broader components: a broad emission component, always in emission and visible only toward the $\ell=59^{\circ}$ sources, and a medium absorption or emission component seen in almost all sources. The broad and medium line components have widths exceeding the line width typical of the more quiescent envelope (e.g., Jørgensen et al. 2002) and are thus likely related to a protostellar outflow.

In the $\ell=59^{\circ}$ sources, both Gaussian components are required to fit the line profile. The medium component has already been observed by Kristensen et al. (2012) toward a sample of low-mass, protostellar objects, by van der Tak et al. (2013) toward a sample of high-mass, star-forming regions, and also by Herpin et al. (2012) toward the massive protostar W43MM1. Figure 6 shows that the medium component in most cases is centered at the source velocity (offset $\lesssim 1 \mathrm{~km} \mathrm{~s}^{-1}$ ), with $V_{\text {med }}\left(\mathrm{H}_{2} \mathrm{O}\right)-V_{\text {lsr }}\left(\mathrm{N}_{2} \mathrm{H}^{+}\right)$having positive values in almost all cases. We already noted in the previous section that the narrow component is blueshifted relative to the medium component. van der Tak et al. (2013) found that their medium component, which they call "narrow outflow component", usually appears in absorption, and is instead blueshifted relative to the narrow component, which they call "envelope component". However, their decomposition of the line profiles is based on higher excitation water lines.

Both Kristensen et al. (2012) and van der Tak et al. (2013) associate the origin of the medium component to the region, closer to the central protostar, where the gas is currently being shocked, whereas they associate the broader component with the sweptup gas of the molecular outflow. However, Herpin et al. (2012) report that their medium velocity component is likely due to a combination of turbulence and infall. A more detailed analysis of the different velocity components in shocked molecular gas has been carried out, for example, by Busquet et al. (2014) in the $\mathrm{L} 1157-\mathrm{B} 1$ shock region. They performed a multiline $\mathrm{H}_{2} \mathrm{O}$ survey probing a wide range of excitation conditions. In addition to the component in absorption (probably due to the photodesorption of icy mantles at the edge of the cloud), Busquet et al. (2014) found two gas components, connected with a J-type shock associated with a jet and with different excitation conditions. The first component is a relatively extended $\left(\sim 10^{\prime \prime}\right.$ corresponding to $\left.\sim 2 \times 10^{4} \mathrm{AU}\right)$, warm $(\sim 200 \mathrm{~K})$, and dense $\left(\sim 10^{6} \mathrm{~cm}^{-3}\right)$ region, and the second is a more compact (less than $5^{\prime \prime}$ or $\sim 10^{4} \mathrm{AU}$ ), hot $(\sim 1000 \mathrm{~K})$ and tenuous $\left(\sim 10^{3}-10^{4} \mathrm{~cm}^{-3}\right)$ component. The water abundance is very high in the hot gas $\left(\sim 10^{-4}\right)$ and it decreases down to $\sim 10^{-6}$ in the warm component. Both warm and hot components have their peak at low velocities, with the hot region dominating the total flux at the high end of the velocity range.
In our work, and with only the data presented here, it is not possible to infer the exact physical origin of the medium component of the $\mathrm{H}_{2} \mathrm{O}\left(1_{10}-1_{01}\right)$ transition. However, the correlation between the line widths of the broad and medium components in the $\ell=59^{\circ}$ sources, shown in the bottom panel of Fig. 5, suggests an outflow or shock origin for the medium component as well. In addition, Kristensen et al. (2012) speculate that the phenomenon producing the masers should also have a thermal component associated with it, which then produces the medium component. We analyze the association with masers in Sect. 4.3.

As far as sources in the $\ell=30^{\circ}$ region are concerned, no broad component has been detected. Observationally, this may be a consequence of the larger distances of these sources compared to the $\ell=59^{\circ}$ region. Alternatively, the viewing angle of the molecular outflows and/or shocks may also determine whether a source shows a broad, or both a broad and medium component. Because of the small sample size of $\ell=30^{\circ}$ sources, this kind of effect could be statistically significant, although in the $\ell=59^{\circ}$ region, with a comparable sample size, all sources show a broad component. An alternative, more physical reason for the lack of a broad component toward the $\ell=30^{\circ}$ region could be a different evolutionary phase (see Sect. 4.4).

As far as $\mathrm{NH}_{3}$ and $\mathrm{N}_{2} \mathrm{H}^{+}$are concerned, the line width of the ammonia line is likely affected by the presence of molecular outflows. In particular, source 159-441 is the only one where the $\mathrm{NH}_{3}$ profile shows the same three components as the water line. While the water narrow component is generally blueshifted relative to the medium and broad components, all ammonia components have remarkably similar velocities. Three more sources show a $\mathrm{NH}_{3}$ narrow and medium component (or two narrow components): 159-442, 159-444, and 130-304. The two ammonia components also have very similar velocities in $159-442$. In the other two sources, 159-444, and 130-304, however, inverse P-Cygni profiles are observed, and are discussed in the next section.

\subsubsection{Regular and inverse P-Cygni profiles}

In a total of three cases, we observed a regular or inverse P-Cygni profile, which are usually associated with envelope expansion and infall, respectively. An inverse P-Cygni profile is characterized by an absorption feature that is redshifted relative to an emission feature, and is typically caused by a velocity and excitation gradient in the gas falling in toward a central object. Two sources have inverse P-Cygni profiles, 130-304 and 159-444. In the case of source 159-444, the inverse P-Cygni profile is visible in the spectrum of water as superposed on the broad outflow component. In this source, a redshifted absorption is also visible in the ammonia spectrum. Toward 130-304 the inverse P-Cygni profile is only detected in the $\mathrm{NH}_{3}$ spectrum, whereas the water spectrum shows multiple absorption lines from the diffuse interstellar gas, and at the source systemic velocity the water line profile appears almost completely self-absorbed (Sect. 3.4.2). A recent example of multiple ammonia lines, observed with Herschel toward the massive star-forming region $\mathrm{G} 34.3+0.15$ and showing inverse P-Cygni profiles, can be found in Hajigholi et al. (2015). The only regular P-Cygni profile we have detected, toward source 130-512, is also superposed on what looks like an outflow/shock component. These three profiles are thus all different at some level and suggest a great variety of kinematic and excitation conditions.

To give the simplest possible description of a contracting or expanding system that can reproduce the observed line profiles, Di Francesco et al. (2001) used a simple model consisting of 
Table 6. Best-fit parameters of the infall/outflow modeling.

\begin{tabular}{|c|c|c|c|c|c|c|c|c|c|}
\hline Source & Species & $\begin{array}{c}V_{\mathrm{in}} \\
{\left[\mathrm{km} \mathrm{s}^{-1}\right]}\end{array}$ & $\begin{array}{c}\sigma \\
{\left[\mathrm{km} \mathrm{s}^{-1}\right]}\end{array}$ & $\begin{array}{c}V_{\mathrm{lsr}^{a}}{ }^{-1} \\
{\left[\mathrm{~km} \mathrm{~s}^{-1}\right]}\end{array}$ & $\tau_{\mathrm{o}}$ & $\Phi$ & $\begin{array}{l}T_{\mathrm{c}}{ }^{a} \\
{[\mathrm{~K}]}\end{array}$ & $\begin{array}{l}T_{\mathrm{f}}{ }^{a} \\
{[\mathrm{~K}]}\end{array}$ & $\begin{array}{c}T_{\mathrm{r}} \\
{[\mathrm{K}]}\end{array}$ \\
\hline $159-444$ & $\mathrm{H}_{2} \mathrm{O}$ & 0.5 & 1.5 & 34.3 & 1.7 & 0.14 & 37.0 & 4.0 & 17.2 \\
\hline $130-304$ & $\mathrm{NH}_{3}$ & 2.5 & 1.7 & 90.7 & 3.1 & 0.01 & 350.0 & 4.0 & 10.5 \\
\hline $130-512^{b}$ & $\mathrm{H}_{2} \mathrm{O}$ & -2.1 & 1.6 & 61.4 & 1.3 & 0.01 & 63.0 & 4.0 & $11.0^{a}$ \\
\hline
\end{tabular}

Notes. See text for a description of the parameters used in the fit. ${ }^{(a)}$ Fixed parameter, see text. ${ }^{(b)}$ Tentative result.

two slabs moving toward each other with a continuum source between them. This model was a modification of a previous "twolayer" code used by Myers et al. (1996). This model is appealing because of its simplicity, but on the other hand it gives only the crudest picture of the optical depth and excitation temperature of the absorbing and emitting gas. In addition, the model uses a relatively large number of free parameters (eight in total, if none is held fixed), including the excitation temperature of the front and rear layers and the continuum source $\left(T_{\mathrm{f}}, T_{\mathrm{r}}, T_{\mathrm{c}}\right)$ the filling factor of the absorbing source with respect to the telescope beam $(\Phi)$, the peak optical depth of the front and rear layers $\left(\tau_{\mathrm{o}}\right)$, the turbulent velocity dispersion in each layer $(\sigma)$, the infall velocity $\left(V_{\text {in }}\right)$, and the systemic velocity $\left(V_{\mathrm{lsr}}\right)$.

Therefore, applying the simple two-slab model described above to the two sources with inverse P-Cygni profiles, we obtain the best-fit infall models shown in Fig. 7, with the corresponding best-fit parameters listed in Table 6. In the case of source 159-444, the broad outflow component and an additional narrow absorption feature, blueshifted with respect to the inverse P-Cygni profile (see Table 3), have been subtracted from the original spectrum before applying the model. For both sources, we fixed the value of $T_{\mathrm{c}}$ to that shown in Table 2, and we fixed the value of $V_{\text {lsr }}$ to the velocity of $\mathrm{N}_{2} \mathrm{H}^{+}$(see Table 3). In Sect. 4.2.3 we suggested that the gas front layer may have a low excitation temperature and thus we fixed it to the approximate intermediate value $\left(T_{\mathrm{f}} \simeq 4 \mathrm{~K}\right)$ shown by Myers et al. (1996) in their Fig. 1. In comparison, Hajigholi et al. (2015) find an excitation temperature of $\simeq 7-8 \mathrm{~K}$ in the absorbing envelope of source G34.3+0.15.

Through the comparison of different infall model profiles with the observations, we find that $V_{\text {in }}$ and $\sigma$ can vary by as much as $0.2-0.5 \mathrm{~km} \mathrm{~s}^{-1}$, and are not very sensitive to $T_{\mathrm{f}}$. However, these tests do not take into account the uncertainty in the beamfilling factor, used to convert the antenna temperature to line brightness temperature (see Sect. 3.4), which may also affect the best-fit results. Thus, our results show that while the infall velocity toward $159-444$ is comparable to the values found by Di Francesco et al. (2001) and Kristensen et al. (2012, who analyze low-mass protostars), the values of $\sigma$ (and the value of $V_{\text {in }}$ toward 130-304) are somewhat higher, since these authors find $V_{\text {in }}, \sigma \lesssim 1 \mathrm{~km} \mathrm{~s}^{-1}$. Our estimated values of $\sigma$ are consistent with those found by Hajigholi et al. (2015) toward G34.3+0.15. Some of the high-mass protostars studied by van der Tak et al. (2013) also show regular and inverse P-Cygni profiles, but they do not analyze the line profiles and thus we cannot directly compare our results. Instead, we can compare our results with those of Herpin et al. (2012) toward the massive protostar W43-MM1, where they derive infall velocities as high as $2.9 \mathrm{~km} \mathrm{~s}^{-1}$ and turbulent velocities $\gtrsim 2 \mathrm{~km} \mathrm{~s}^{-1}$. These values are much more similar to our estimates of $\sigma$ and of the infall velocity toward 130-304.

As far as the only source, 130-512, with a regular P-Cygni profile in our sample, this source shows a narrow absorption component at a velocity of $64 \mathrm{~km} \mathrm{~s}^{-1}$. Therefore, assuming that this additional absorption feature is not related to the overall
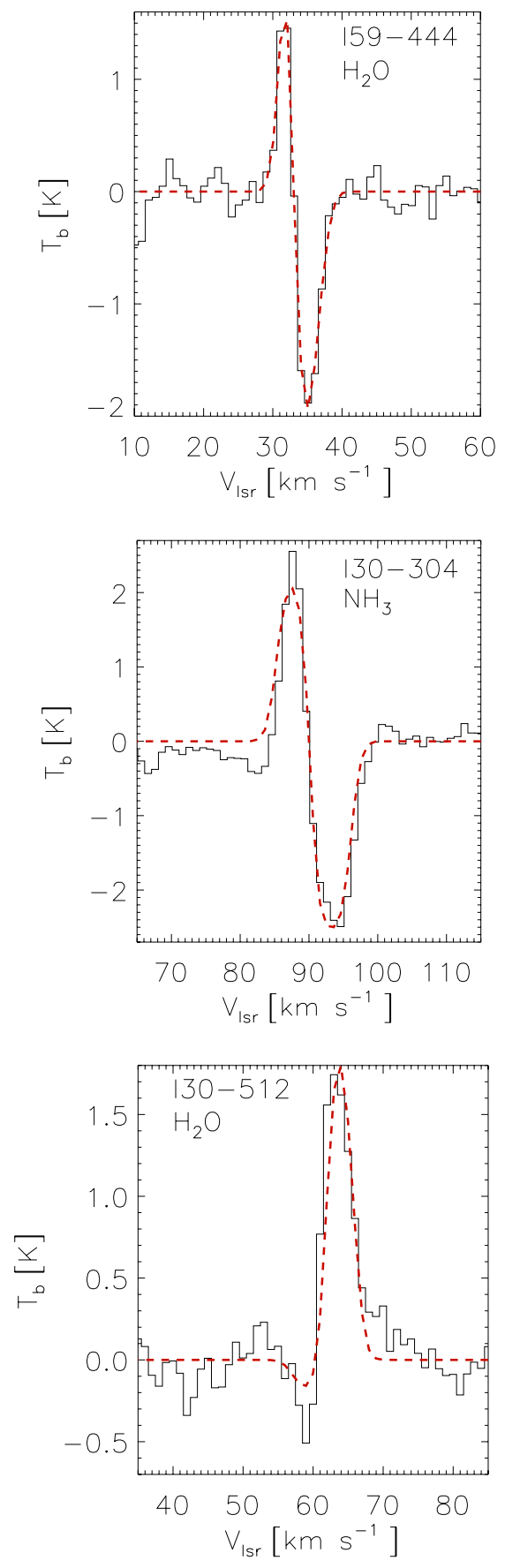

Fig. 7. From top to bottom, inverse P-Cygni profiles detected toward 159-444 $\left(\mathrm{H}_{2} \mathrm{O}\right)$ and 130-304 $\left(\mathrm{NH}_{3}\right)$, and regular P-Cygni profile detected toward 130-512 $\left(\mathrm{H}_{2} \mathrm{O}\right)$. The infall (or expansion, in the case of 130-512) model is shown on top (red, dashed line). The broad outflow component has been subtracted from the spectrum of source 159-444, whereas a narrow absorption component has been added back to the spectrum of $130-512$. 
P-Cygni profile, and to reconstruct the hypothetical P-Cygni profile we added this component back to the water spectrum. The two-layer model described earlier can also be applied to the case of expanding gas, and the only change in the model is that the infall velocity becomes negative. We show our best-fit result in the bottom panel of Fig. 7, obtained by fixing the value of $T_{\mathrm{r}}$, which would otherwise diverge. Clearly, the fit is not very good and it is difficult to determine whether this may be due to the contamination of the line profile, up to the level of possibly resulting in a fake P-Cygni profile. For this reason, our results in Table 6 are labeled as tentative.

While the infall and turbulent velocities are distance independent and, at least in this toy model, do not depend on the mass distribution within the source, determining the mass infall rate would require the knowledge of the characteristic distance of the infall zone or the mass undergoing infall. If the envelopes are in free fall, the velocity at a radius $R_{\text {in }}$ in the envelope is given by $V_{\text {in }}=\sqrt{2 G M_{\text {enc }} / R_{\text {in }}}$, where $M_{\text {enc }}$ is the mass enclosed within the radius $R_{\mathrm{in}}$. Assuming spherical infall symmetry, the mass infall rate can be estimated as (e.g., Kristensen et al. 2012)

$\dot{M}=4 \pi \mu m_{\mathrm{H}} n_{\text {in }}\left(2 G M_{\text {enc }}\right)^{2} V_{\text {in }}^{-3}$,

with $n_{\text {in }}$ the density at the infall radius, $m_{\mathrm{H}}$ and $\mu=2.8$ the hydrogen atom mass and mean molecular weight, respectively. Therefore, to estimate $\dot{M}$ it is necessary to be able to determine either $M_{\text {enc }}$ or the infall radius $R_{\text {in }}$. We do not have a reliable distance determination for source 159-444 (see Table 2), and thus we cannot estimate any mass. In the case of source 130-304, we have a distance estimate and a mass, but the large mass and relatively large distance (see Table 2) suggest that the estimated mass represents a much larger mass than that enclosed within the infalling region. As an example, if we used a typical infall radius estimated by Herpin et al. $(2012)\left(R_{\text {in }} \sim 6 \times 10^{17} \mathrm{~cm}\right)$ then in source 130-304 we would get a mass infall rate $\dot{M} \sim$ $5 \times 10^{-2} M_{\odot} \mathrm{yr}^{-1}$, using as $n_{\text {in }}$ the density given in Table 2 and $V_{\text {in }}$ from Table 6 . Not surprisingly, $\dot{M}$ is comparable to the values estimated by Herpin et al. (2012) toward the massive protostellar clump W43-MM1, and is much higher than the infall rates determined by Kristensen et al. (2012) toward low-mass, protostellar sources.

\subsection{Association with maser emission}

We searched for masers associated with the detected sources (within a 30" radius) and found a total of six sources associated with maser emission: all four detected sources in the $\ell=59^{\circ}$ region and two more in the $\ell=30^{\circ}$ field. Sources 159-441 and 159-445, which are very close, are actually associated with the same methanol maser. All sources, except for 159-442, are associated with a methanol $6.7 \mathrm{GHz}$ maser. Source $159-442$ is the only source associated with a $22 \mathrm{GHz}$ water maser (B193852.5+235736, Palla et al. 1991), while source 130-304 is also associated with an $\mathrm{OH}$ maser.

The methanol masers associated with sources 159-441/ 159-445 and 159-444 were found by comparing our observations with those carried out by Olmi et al. (2014b) toward the $\ell=30^{\circ}$ and $\ell=59^{\circ}$ regions in search of $6.7 \mathrm{GHz} \mathrm{CH} \mathrm{CH}_{3} \mathrm{OH}$ and $6.0 \mathrm{GHz} \mathrm{OH}$ masers. The masers associated with 159-441/ 159-445 and 159-444 are G59.63-0.19 and G59.78+0.63, respectively, and they have peak flux densities of 0.58 and $0.03 \mathrm{Jy}$. As discussed by Olmi et al. (2014b), these two methanol masers belong to a new class of low-brightness masers that could represent an earlier stage of evolution.
The methanol masers have velocities that agree well with the velocities observed in our detected sources (differences are $\left.\$ 1-2 \mathrm{~km} \mathrm{~s}^{-1}\right)$. However, the velocity of the $22 \mathrm{GHz}$ water maser $\left(V_{\mathrm{lsr}} \simeq 50 \mathrm{~km} \mathrm{~s}^{-1}\right)$ associated with source 159-442 significantly differs from the central velocity of any of the Gaussian components of its $\mathrm{H}_{2} \mathrm{O}$ line profile $\left(V_{\mathrm{lsr}} \simeq 35-37 \mathrm{~km} \mathrm{~s}^{-1}\right)$. If we assume that the physical association between these two sources is indeed real, given the small angular separation between them ( $\simeq 4$ arcsec), then one might speculate that the water maser has originated somewhere along the path of the outflow. In fact, the broad Gaussian component in 159-442 has a line width that encompasses the velocity of the water maser.

\subsection{Evolutionary trends}

The relative intensities of the various components used to decompose the line profiles of the water spectra have already been used in an attempt to identify possible evolutionary sequences. For example, Kristensen et al. (2012) associate the disappearance of the broad component with later evolutionary stages in their low-mass protostars sample. van der Tak et al. (2013) then propose that the appearance of the narrow component (or "envelope" component) in emission is an indicator of a warmer envelope, and thus associated with later evolutionary stages. While the narrow component appears in absorption in all $\ell=59^{\circ}$ sources, toward the $\ell=30^{\circ}$ region sources 130-43 and 130-376 are characterized by a narrow component in emission (see Table 3; see also Sect. 3.2 regarding the water detection toward 130-43). In source 130-512, the narrow component is in absorption, but this is a more complex source with a regular P-Cygni profile, as discussed in Sect. 4.2.5.

An alternative method to analyze the evolutionary stages of our sources makes use of the evolutionary tracks previously shown in Fig. 2. These tracks were determined following a method similar to that discussed by Molinari et al. (2008), who proposed an evolutionary sequence for high-mass protostars in terms of two parameters: the envelope mass and the bolometric luminosity. These authors suggest an evolutionary sequence, which is concentrated in two main phases: protostars first accrete mass from their envelopes, and later disperse their envelopes by winds and/or radiation. In Fig. 2 the calculated evolutionary tracks thus rise upward almost vertically in luminosity during the accretion phase, and then proceed horizontally to the left (to lower masses) during the envelope dispersal phase. The positions of our sources in this plot suggest that some (but not all) of the sources with at least one line detection are, for similar ranges of mass, in a more advanced stage of evolution. This is certainly the case for the detected $\ell=59^{\circ}$ sources with a well-defined distance (159-441 and 159-445), while for sources detected toward the $\ell=30^{\circ}$ region a preferential evolutionary phase is not apparent. None of these sources, however, seem to belong to the late phase of envelope dispersal.

There are other indicators that can be used to analyze the relative evolutionary phase of the $\ell=30^{\circ}$ and $\ell=59^{\circ}$ regions. For example, from the discussion about $T_{\mathrm{c}}$ in Sect. 4.1 and Table 5, there is a trend ${ }^{10}$ of higher $T_{\mathrm{c}}$ toward the detected $\ell=30^{\circ}$ sources, suggesting they may be in a more advanced stage of protostellar evolution, compared to sources in the $\ell=59^{\circ} \mathrm{re}-$ gion. Furthermore, as already noted in Sect. 3.3, the typical water line luminosity toward the $\ell=30^{\circ}$ sources is lower compared to that of the $\ell=59^{\circ}$ region. Kristensen et al. (2012)

${ }^{10}$ We use the word "trend" because of the large scatter shown by the $T_{\mathrm{c}}$ values in Table 5 . 
find a similar decrease in water emission between their Class 0 and Class I low-mass protostars. Finally, as stated in Sect. 4.3 two of the $\ell=59^{\circ}$ sources are associated with low-brightness methanol masers, which could indicate an earlier phase of evolution. Source 130-304 is the only source associated with an $\mathrm{OH}$ maser, which is sometimes described as characterizing later evolutionary stages (Breen et al. 2010) compared to $6.7 \mathrm{GHz}$ methanol masers. Therefore, a firm conclusion about the specific evolutionary phase of the observed sample is limited by the small number of sources. However, various indicators tentatively suggest that the detected sources toward the $\ell=30^{\circ}$ region are in a later evolutionary phase compared to those in the $\ell=59^{\circ}$ region.

\section{Summary and conclusions}

Using the HIFI instrument of Herschel we observed the o- $\mathrm{NH}_{3}\left(1_{0}-0_{0}\right) \quad(572 \mathrm{GHz}), \quad 0-\mathrm{H}_{2} \mathrm{O}\left(1_{10}-1_{01}\right) \quad(557 \mathrm{GHz})$ and $\mathrm{N}_{2} \mathrm{H}^{+}(6-5)(559 \mathrm{GHz})$ lines toward a sample of 52 high-mass starless and protostellar clumps selected from the Hi-GAL survey. The target sources are concentrated in two galactic fields, at $\ell=30^{\circ}$ and $\ell=59^{\circ}$, and were observed during the science demonstration phase of Herschel. At least one of the three selected molecular lines was detected in 4 out of 35 sources toward the $\ell=59^{\circ}$ region, and 7 out of 17 objects toward the $\ell=30^{\circ}$ region.

Almost all water spectra can be described as the sum of a narrow $\left(F W H M \sim 3-5 \mathrm{~km} \mathrm{~s}^{-1}\right)$, a medium $\left(F W H M \sim 6-10 \mathrm{~km} \mathrm{~s}^{-1}\right)$ and a broad (up to $F W H M \simeq 30 \mathrm{~km} \mathrm{~s}^{-1}$ ) velocity component. All detected sources of the $\ell=59^{\circ}$ region have a strikingly similar water line profile that is characterized by the broad Gaussian component associated with the presence of molecular outflows, which are also observed in the $\mathrm{NH}_{3}$ line, at least toward one source. Therefore, in these sources there is strong evidence that water line emission is linked to the presence of shocked molecular gas.

The situation is very different in the $\ell=30^{\circ}$ region, since no two profiles are identical and there is no clear detection of a broad component in the water spectra. We find that the continuum temperature, $T_{\mathrm{c}}$, is correlated with the source bolometric luminosity. We also find an indication that the water line luminosity increases with $T_{\mathrm{c}}$. The detected sources in the $\ell=59^{\circ}$ region have both a higher water line luminosity and water abundance, compared to sources detected in the $\ell=30^{\circ}$ region. Although we have few sources, this trend constitutes additional support to a scenario where the water abundance is linked to shocks.

In terms of the gas kinematics, the general appearance in absorption of the narrow Gaussian component indicates a high $\mathrm{H}_{2} \mathrm{O}$ column density and a low excitation temperature, suggesting that the absorption is being caused by the outer envelope and ambient cloud. The correlation between the line widths of the broad and medium components in the $\ell=59^{\circ}$ sources suggests an outflow or shock origin for the medium component. In addition, we also detect in a few sources inverse and regular P-Cygni profiles, although we do not find any link with other source parameters. A firm conclusion about the specific evolutionary phase of the observed sample is limited by the small number of sources. However, various indicators tentatively suggest that the detected sources toward the $\ell=30^{\circ}$ region are in a later evolutionary phase compared to those in the $\ell=59^{\circ}$ region.

Acknowledgements. HIFI was designed and built by a consortium of institutes and university departments from across Europe, Canada, and the USA under the leadership of SRON Netherlands Institute for Space Research, Groningen, The Netherlands and with major contributions from Germany, France and the USA. Consortium members are: Canada: CSA, U.Waterloo; France: CESR, LAB, LERMA, IRAM; Germany: KOSMA, MPIfR, MPS; Ireland, NUI Maynooth; Italy: ASI, IAPS-INAF, Osservatorio Astrofisico di Arcetri-INAF; Netherlands: SRON, TUD; Poland: CAMK, CBK; Spain: Observatorio Astronomico Nacional (IGN), Centro de Astrobiologia (CSIC-INTA). Sweden: Chalmers University of Technology - MC2, RSS \& GARD; Onsala Space Observatory; Swedish National Space Board, Stockholm University - Stockholm Observatory; Switzerland: ETH Zurich, FHNW; USA: Caltech, JPL, NHSC. CMP acknowledges generous support from the Swedish National Space Board.

\section{References}

Bergin, E. A., \& Tafalla, M. 2007, ARA\&A, 45, 339

Bontemps, S., Motte, F., Csengeri, T., \& Schneider, N. 2010, A\&A, 524, A18

Breen, S. L., Caswell, J. L., Ellingsen, S. P., \& Phillips, C. J. 2010, MNRAS, 406, 1487

Busquet, G., Lefloch, B., Benedettini, M., et al. 2014, A\&A, 561, A120

Caselli, P., Keto, E., Bergin, E. A., et al. 2012, ApJ, 759, L37

de Graauw, T., Helmich, F. P., Phillips, T. G., et al. 2010, A\&A, 518, L6

Di Francesco, J., Myers, P. C., Wilner, D. J., Ohashi, N., \& Mardones, D. 2001, ApJ, 562, 770

Elia, D., Schisano, E., Molinari, S., et al. 2010, A\&A, 518, L97

Green, J. A., \& McClure-Griffiths, N. M. 2011, MNRAS, 417, 2500

Hajigholi, M., Persson, C. M., Wirström, E. S., et al. 2015, A\&A, in press, DOI: 10.1051/0004-6361/201526451

Herpin, F., Chavarría, L., van der Tak, F., et al. 2012, A\&A, 542, A76

Ho, P. T. P., \& Townes, C. H. 1983, ARA\&A, 21, 239

Jackson, J. M., Rathborne, J. M., Shah, R. Y., et al. 2006, ApJS, 163, 145

Jørgensen, J. K., Schöier, F. L., \& van Dishoeck, E. F. 2002, A\&A, 389, 908

Kristensen, L. E., \& van Dishoeck, E. F. 2011, Astron. Nachr., 332, 475

Kristensen, L. E., van Dishoeck, E. F., Bergin, E. A., et al. 2012, A\&A, 542, A8

Larson, R. B. 1981, MNRAS, 194, 809

Leurini, S., Gusdorf, A., Wyrowski, F., et al. 2014, A\&A, 564, L11

Marseille, M. G., van der Tak, F. F. S., Herpin, F., \& Jacq, T. 2010, A\&A, 522, A40

Molinari, S., Pezzuto, S., Cesaroni, R., et al. 2008, A\&A, 481, 345

Molinari, S., Swinyard, B., Bally, J., et al. 2010, PASP, 122, 314

Motte, F., Bontemps, S., Schilke, P., et al. 2007, A\&A, 476, 1243

Myers, P. C., Mardones, D., Tafalla, M., Williams, J. P., \& Wilner, D. J. 1996, ApJ, 465, L133

Netterfield, C. B., Ade, P. A. R., Bock, J. J., et al. 2009, ApJ, 707, 1824

Olmi, L., Ade, P. A. R., Anglés-Alcázar, D., et al. 2009, ApJ, 707, 1836

Olmi, L., Araya, E. D., Chapin, E. L., et al. 2010, ApJ, 715, 1132

Olmi, L., Anglés-Alcázar, D., Elia, D., et al. 2013, A\&A, 551, A111

Olmi, L., Anglés-Alcázar, D., Elia, D., et al. 2014a, A\&A, 564, A87

Olmi, L., Araya, E. D., Hofner, P., et al. 2014b, A\&A, 566, A18

Palla, F., Brand, J., Comoretto, G., Felli, M., \& Cesaroni, R. 1991, A\&A, 246, 249

Persson, C. M., Olberg, M., Hjalmarson, Å., et al. 2009, A\&A, 494, 637

Pilbratt, G. L., Riedinger, J. R., Passvogel, T., et al. 2010, A\&A, 518, L1

Rieke, G. H., Young, E. T., Engelbracht, C. W., et al. 2004, ApJS, 154, 25

Roelfsema, P. R., Helmich, F. P., Teyssier, D., et al. 2012, A\&A, 537, A17

Russeil, D. 2003, A\&A, 397, 133

Russeil, D., Zavagno, A., Motte, F., et al. 2010, A\&A, 515, A55

Russeil, D., Pestalozzi, M., Mottram, J. C., et al. 2011, A\&A, 526, A151

Schneider, N., Bontemps, S., Simon, R., et al. 2011, A\&A, 529, A1

Tan, J. C., Beltrán, M. T., Caselli, P., et al. 2014, Protostars and Planets VI, 149

van der Tak, F. F. S., Black, J. H., Schöier, F. L., Jansen, D. J., \& van Dishoeck, E. F. 2007, A\&A, 468, 627

van der Tak, F. F. S., Chavarría, L., Herpin, F., et al. 2013, A\&A, 554, A83

van Dishoeck, E. F., Kristensen, L. E., Benz, A. O., et al. 2011, PASP, 123, 138

Wirström, E. S., Charnley, S. B., Persson, C. M., et al. 2014, ApJ, 788, L32

Zinnecker, H., \& Yorke, H. W. 2007, ARA\&A, 45, 481 
Appendix A: Spectra of sources in the $\ell=30^{\circ}$ region

All spectra are in $T_{\mathrm{A}}^{*}$ units and have been smoothed to a spectral resolution of $1 \mathrm{~km} \mathrm{~s}^{-1}$, unless noted otherwise in the caption.

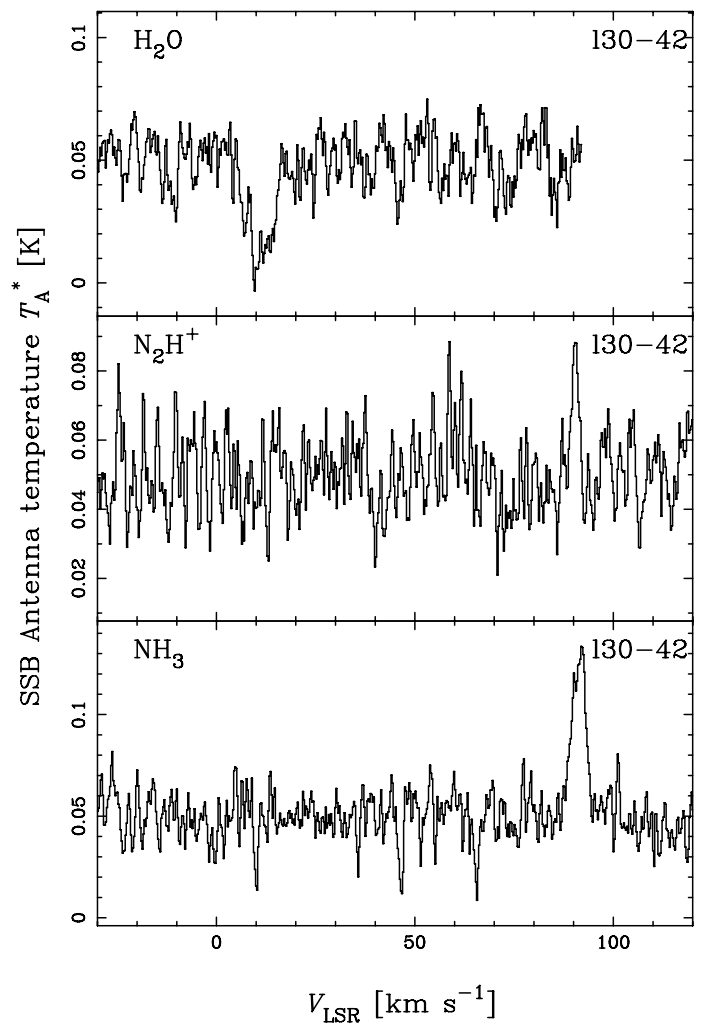

Fig. A.1. Original channel width $\left(0.27 \mathrm{~km} \mathrm{~s}^{-1}\right)$.

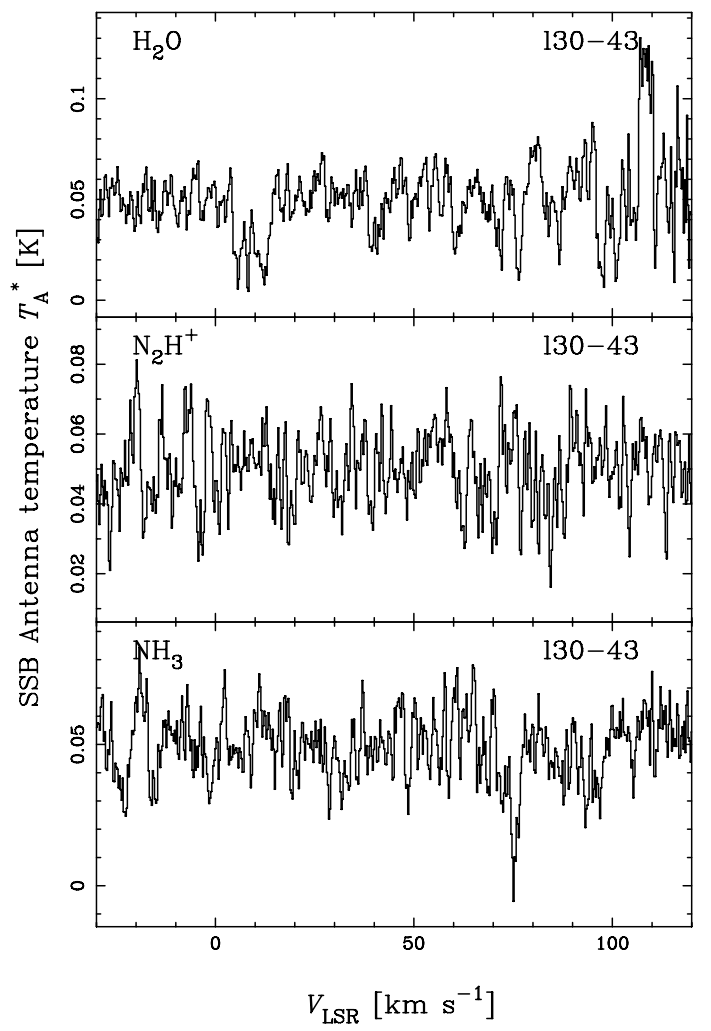

Fig. A.2. Original channel width $\left(0.27 \mathrm{~km} \mathrm{~s}^{-1}\right)$.

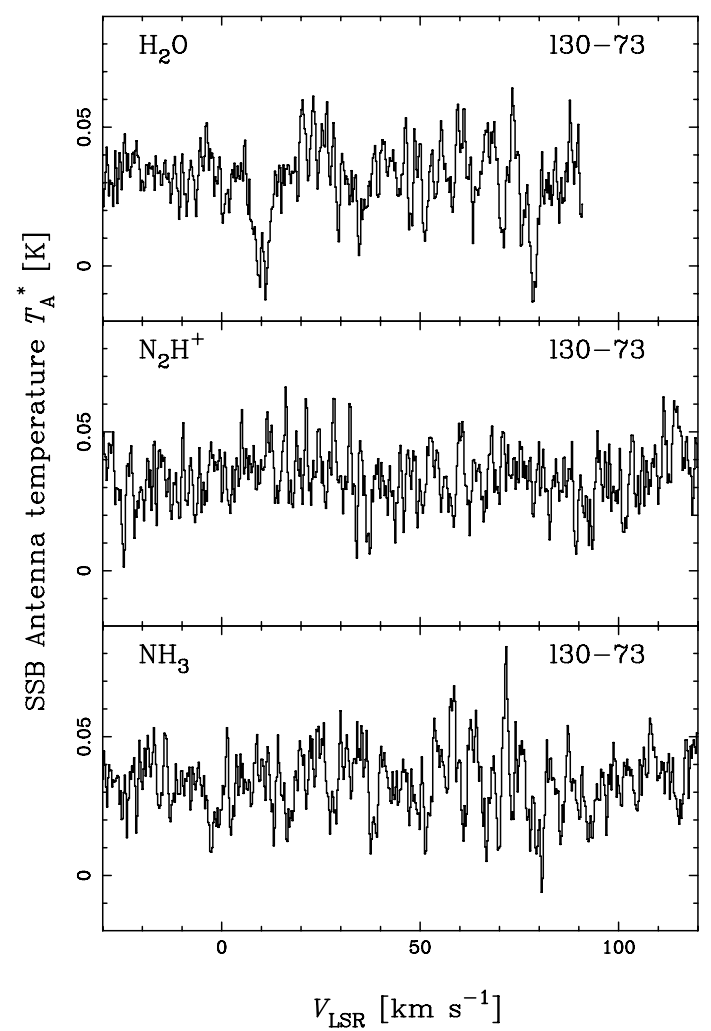

Fig. A.3. Original channel width $\left(0.27 \mathrm{~km} \mathrm{~s}^{-1}\right)$.

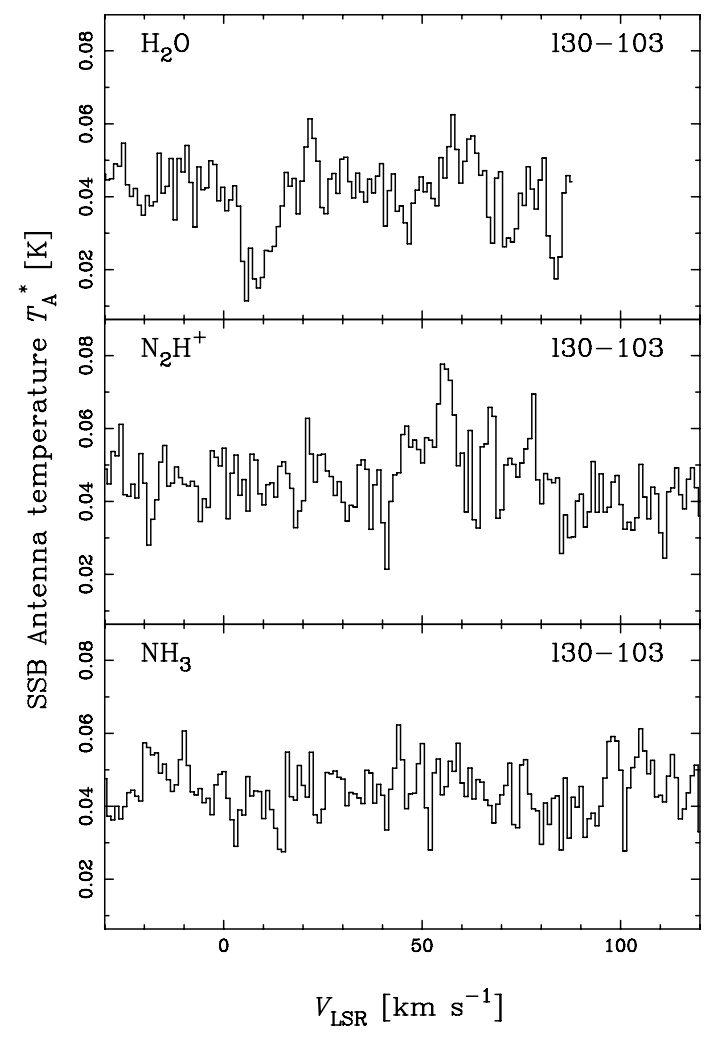

Fig. A.4. 
L. Olmi et al.: High-mass starless and protostellar clumps identified by the Hi-GAL survey

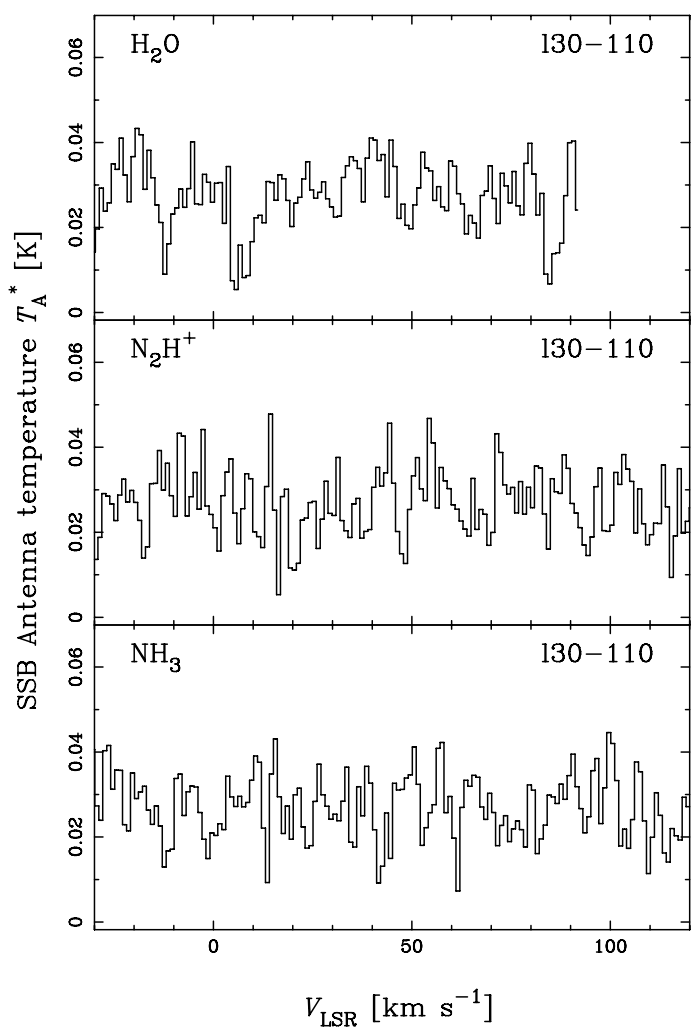

Fig. A.5.

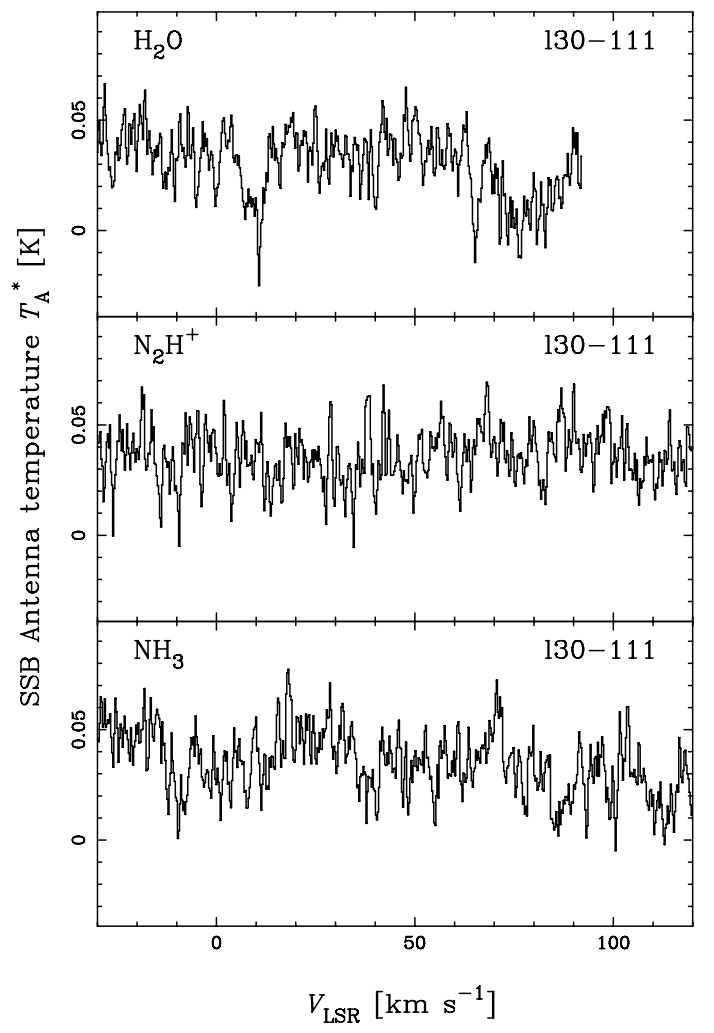

Fig. A.6. Original channel width $\left(0.27 \mathrm{~km} \mathrm{~s}^{-1}\right)$.

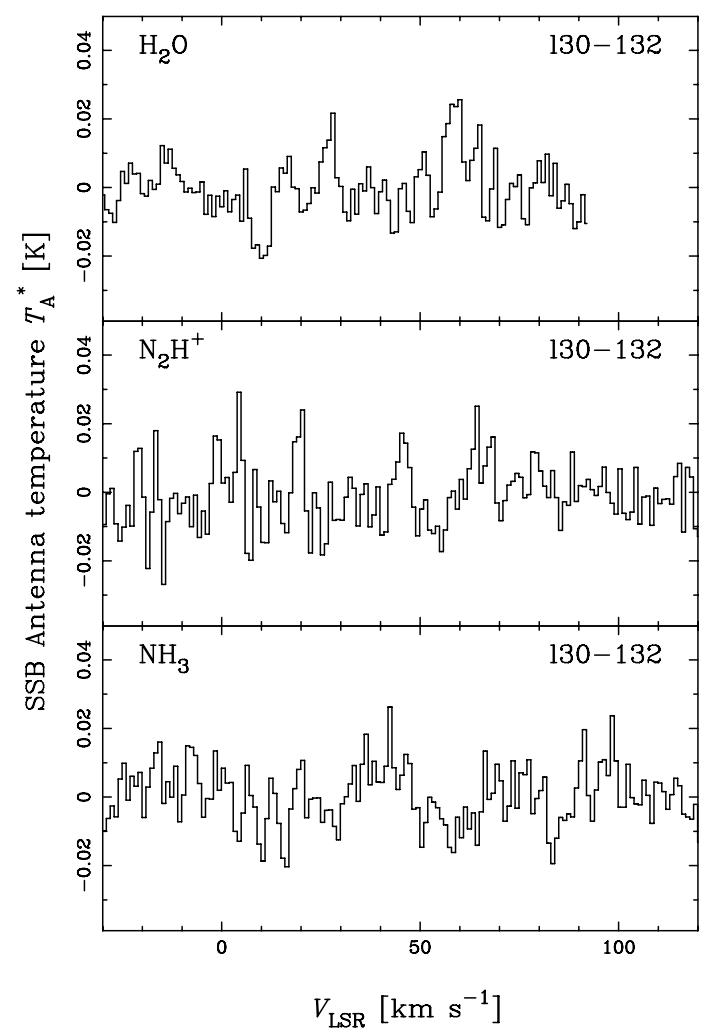

Fig. A.7.

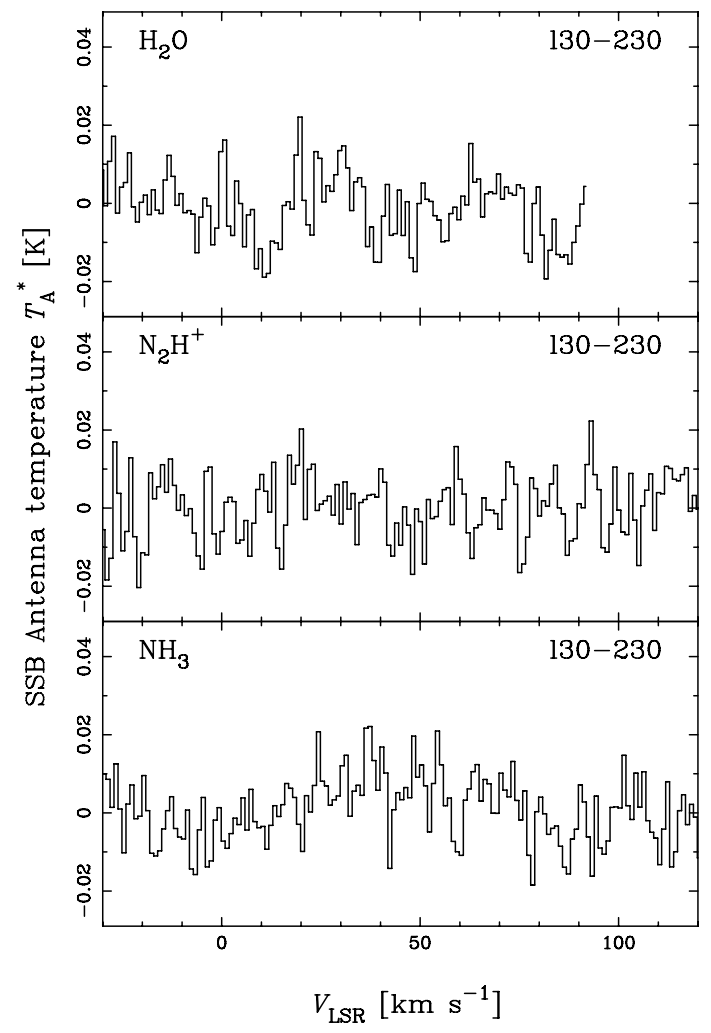

Fig. A.8. 


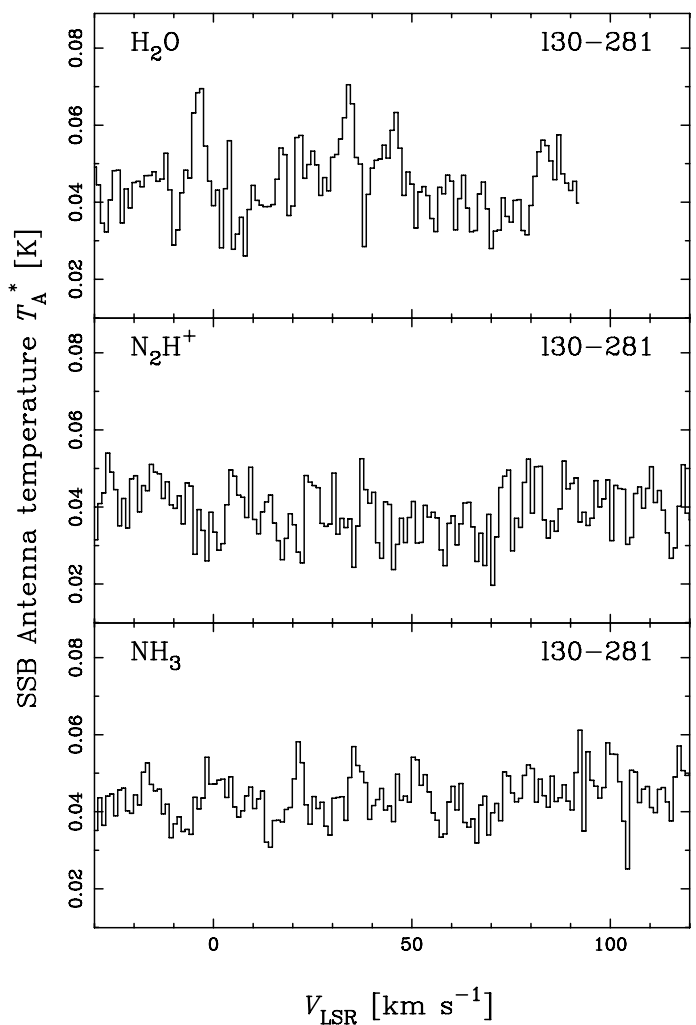

Fig. A.9.

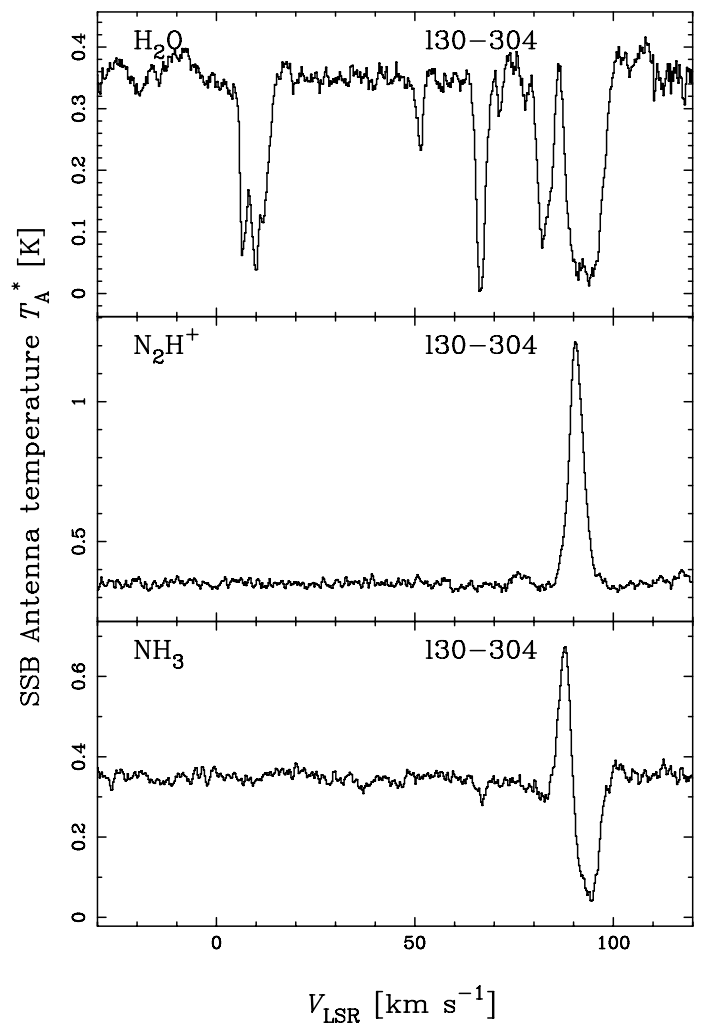

Fig. A.10. Original channel width $\left(0.27 \mathrm{~km} \mathrm{~s}^{-1}\right)$.

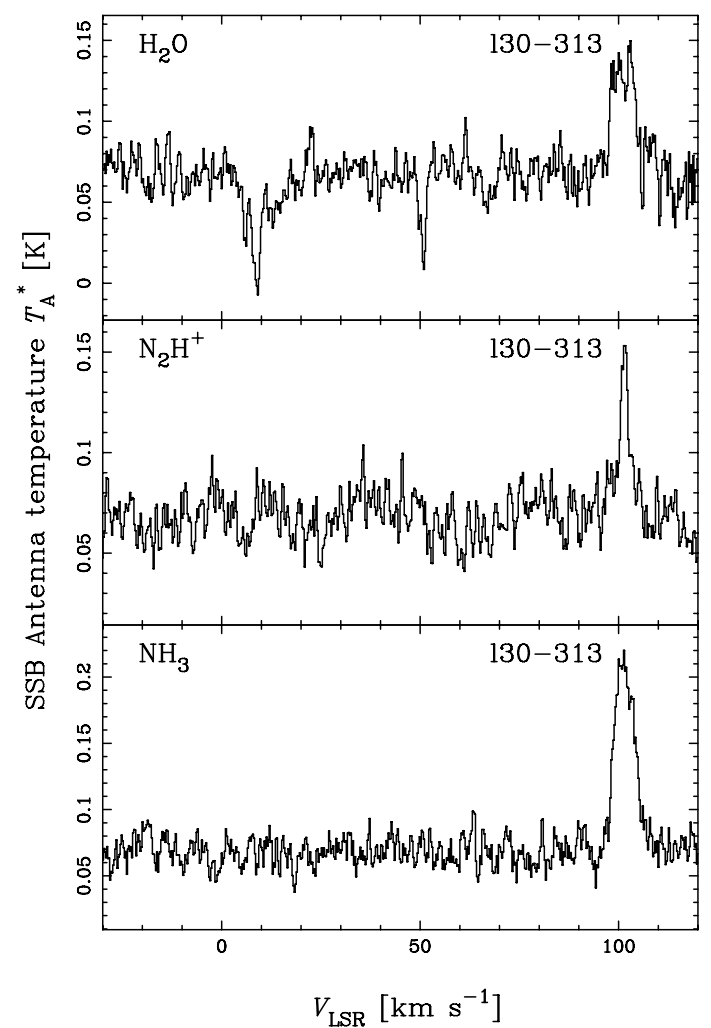

Fig. A.11. Original channel width $\left(0.27 \mathrm{~km} \mathrm{~s}^{-1}\right)$.

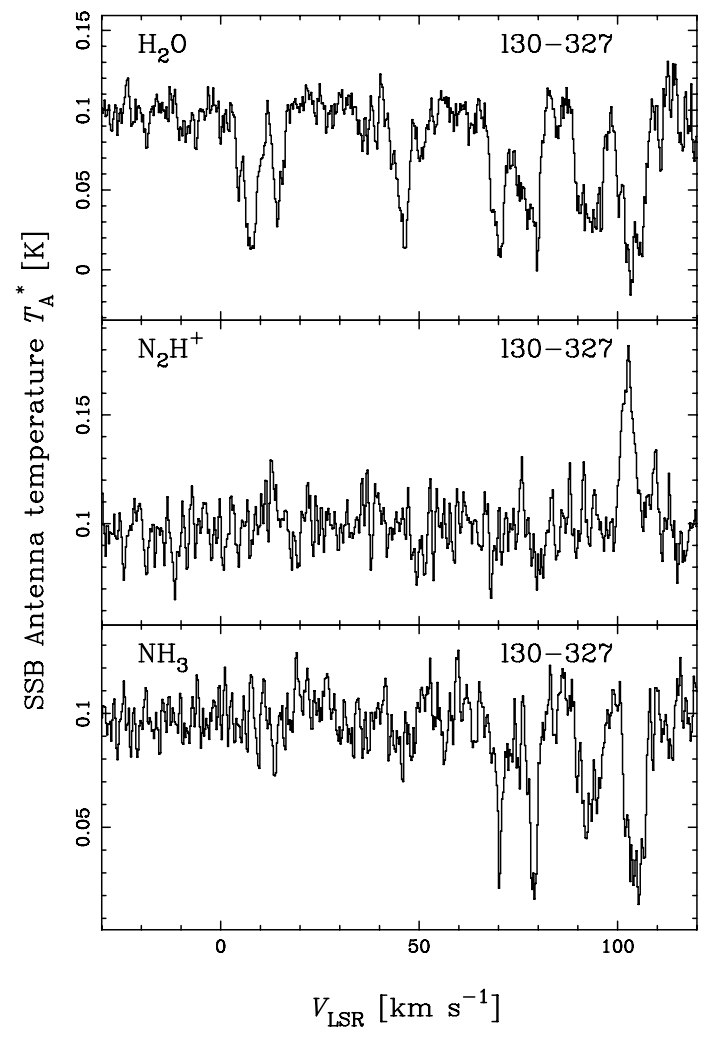

Fig. A.12. Original channel width $\left(0.27 \mathrm{~km} \mathrm{~s}^{-1}\right)$. 
L. Olmi et al.: High-mass starless and protostellar clumps identified by the Hi-GAL survey

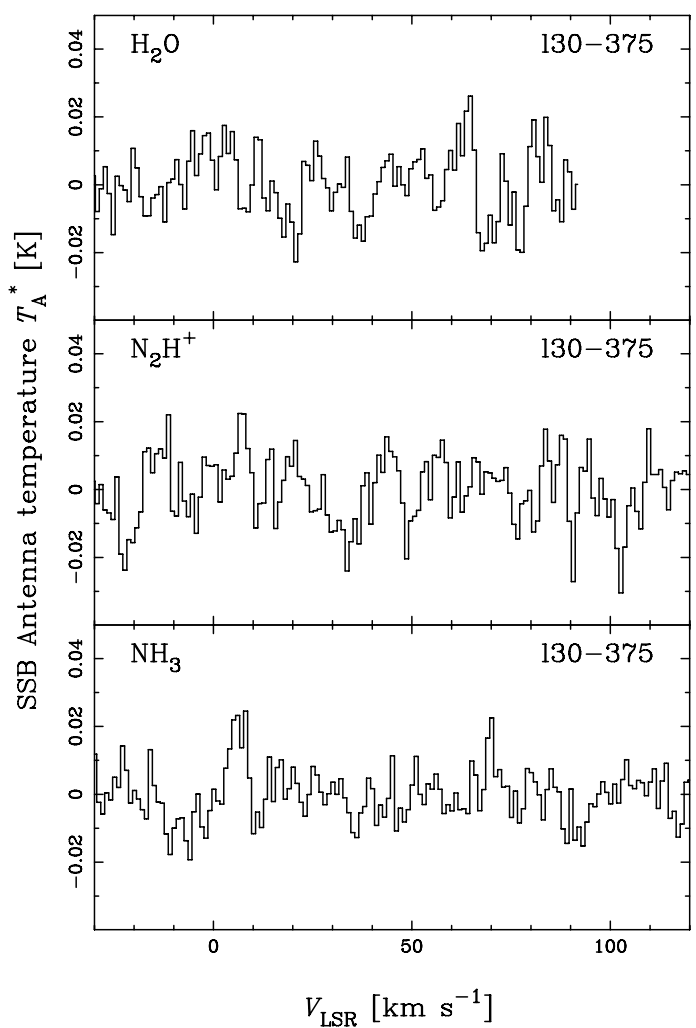

Fig. A.13.

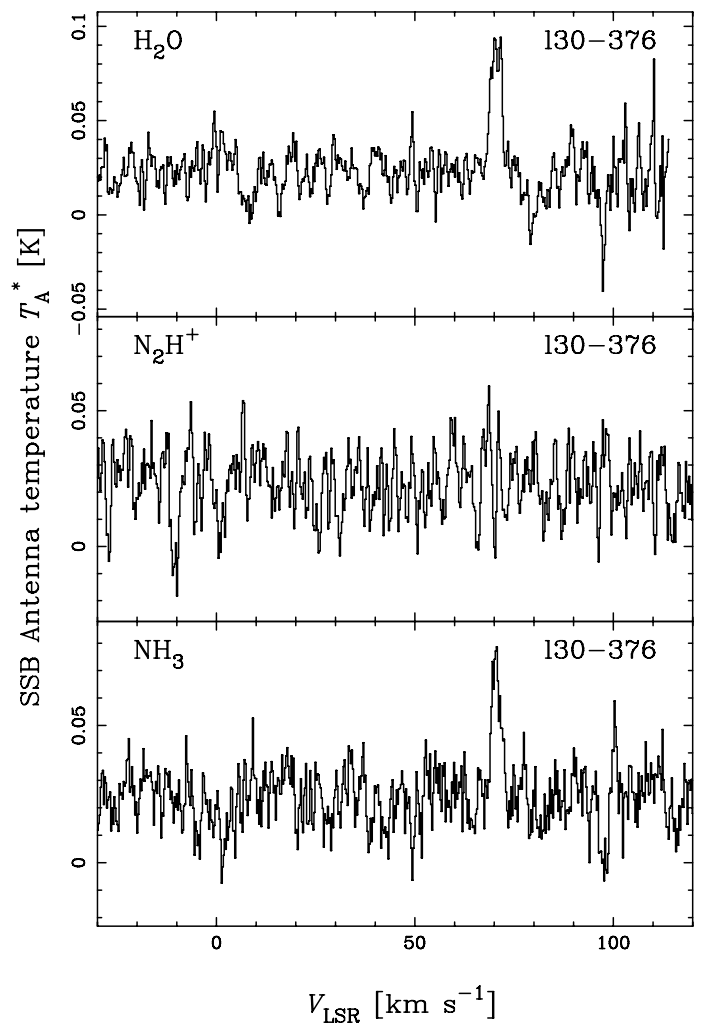

Fig. A.14. Original channel width $\left(0.27 \mathrm{~km} \mathrm{~s}^{-1}\right)$.

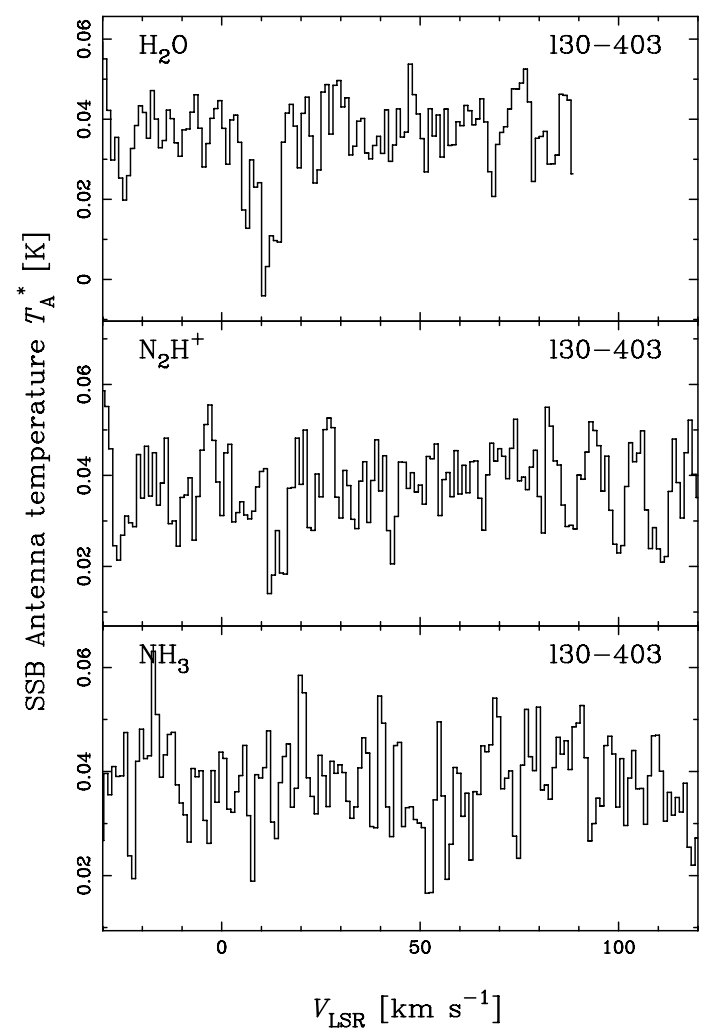

Fig. A.15.

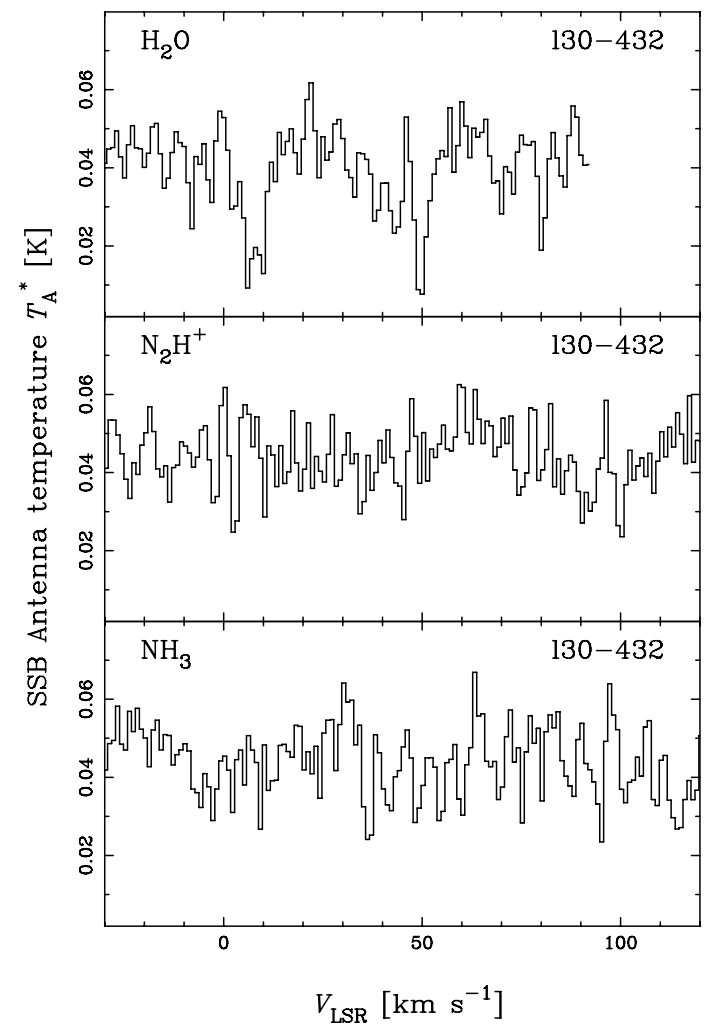

Fig. A.16. 


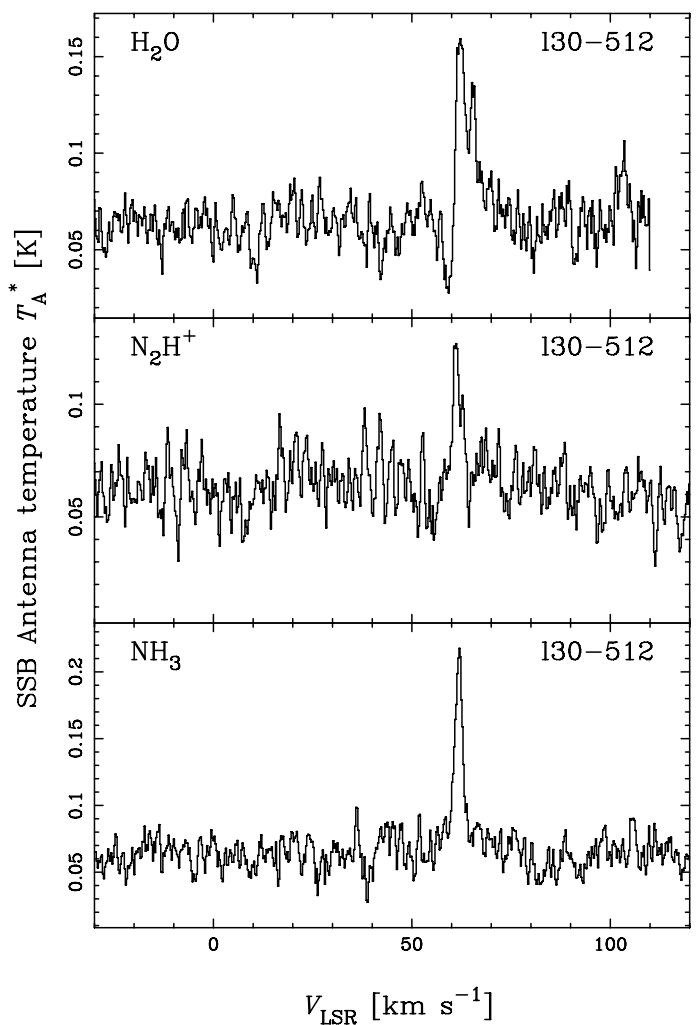

Fig. A.17. Original channel width $\left(0.27 \mathrm{~km} \mathrm{~s}^{-1}\right)$.

\section{Appendix B: Spectra of sources in the $\ell=59^{\circ}$} region

All spectra are in $T_{\mathrm{A}}^{*}$ units and have been smoothed to a spectral resolution of $1 \mathrm{~km} \mathrm{~s}^{-1}$, unless noted otherwise in the caption.

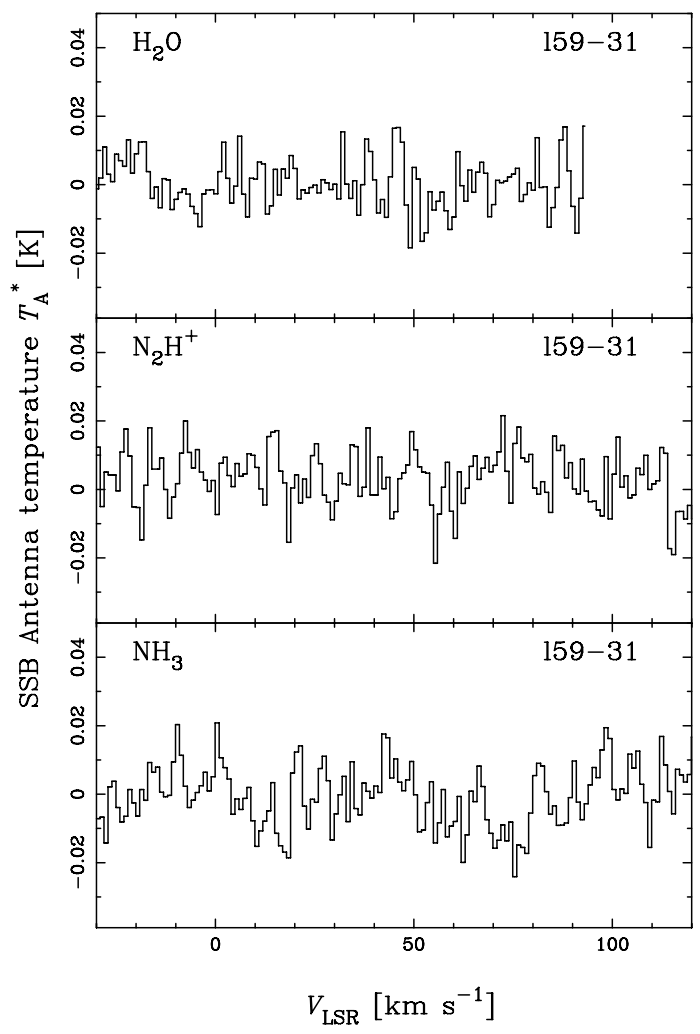

Fig. B.1.

A125, page 20 of 28

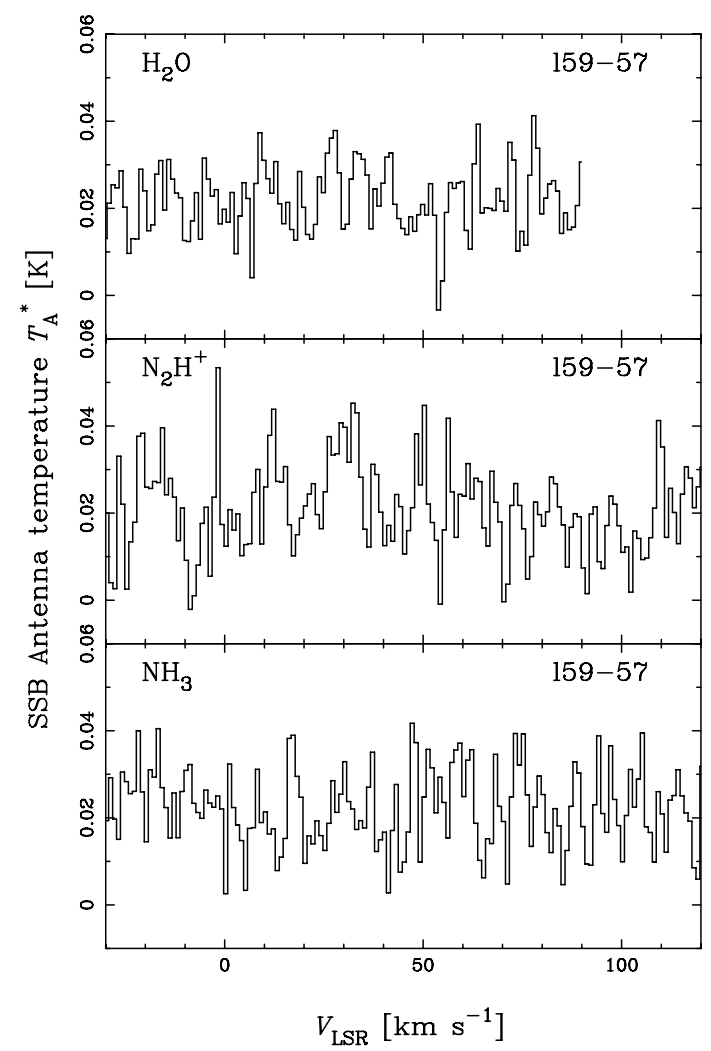

Fig. B.2.

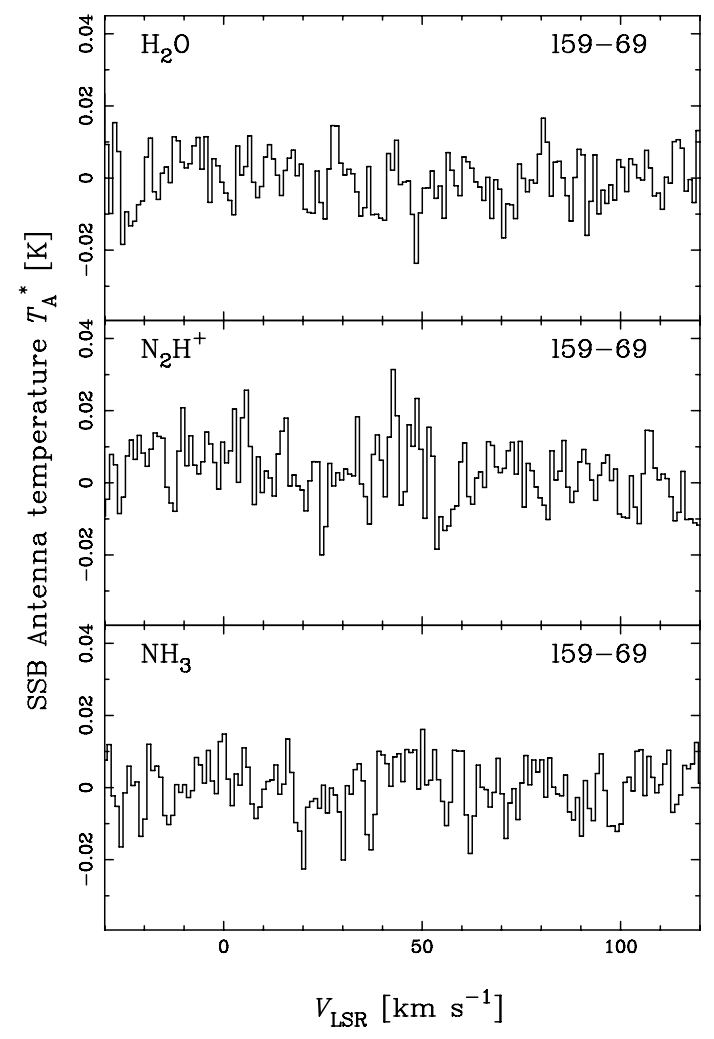

Fig. B.3. 
L. Olmi et al.: High-mass starless and protostellar clumps identified by the Hi-GAL survey

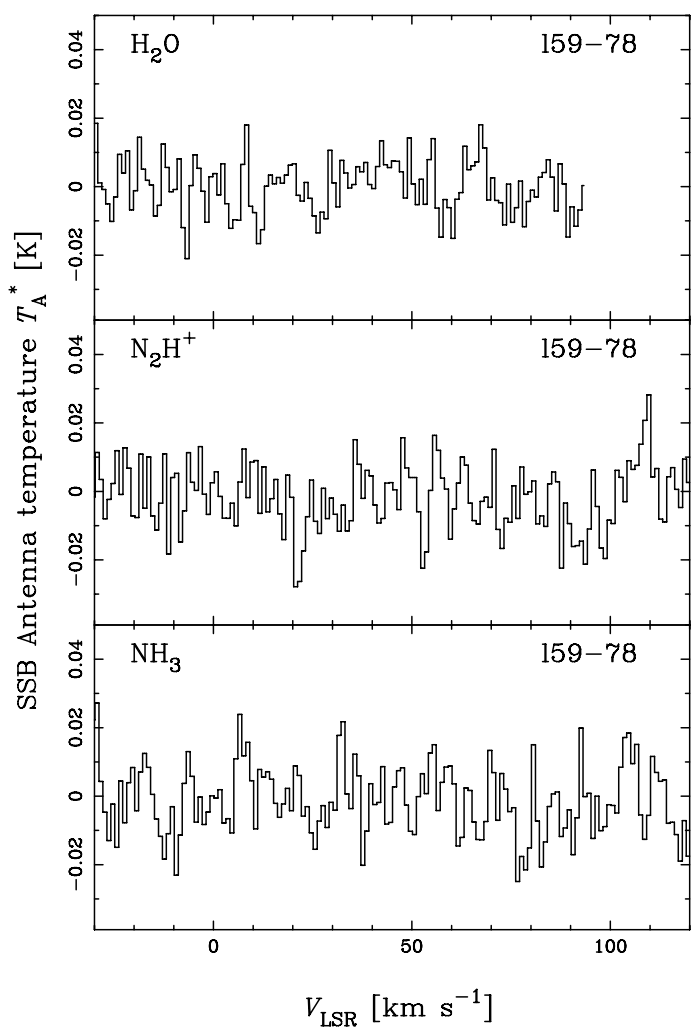

Fig. B.4.

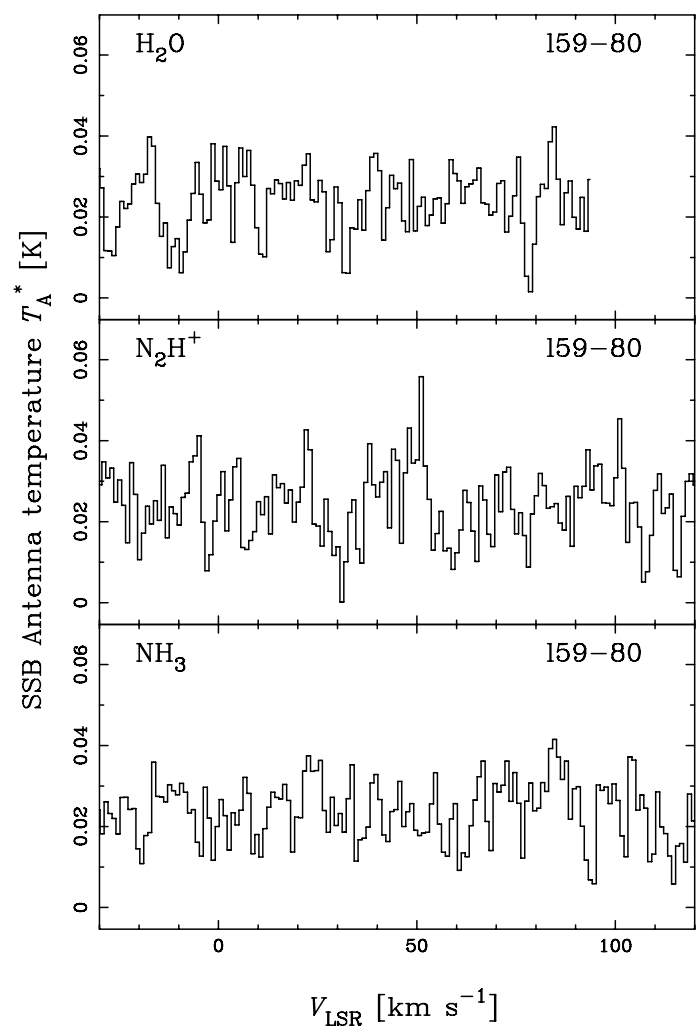

Fig. B.5.

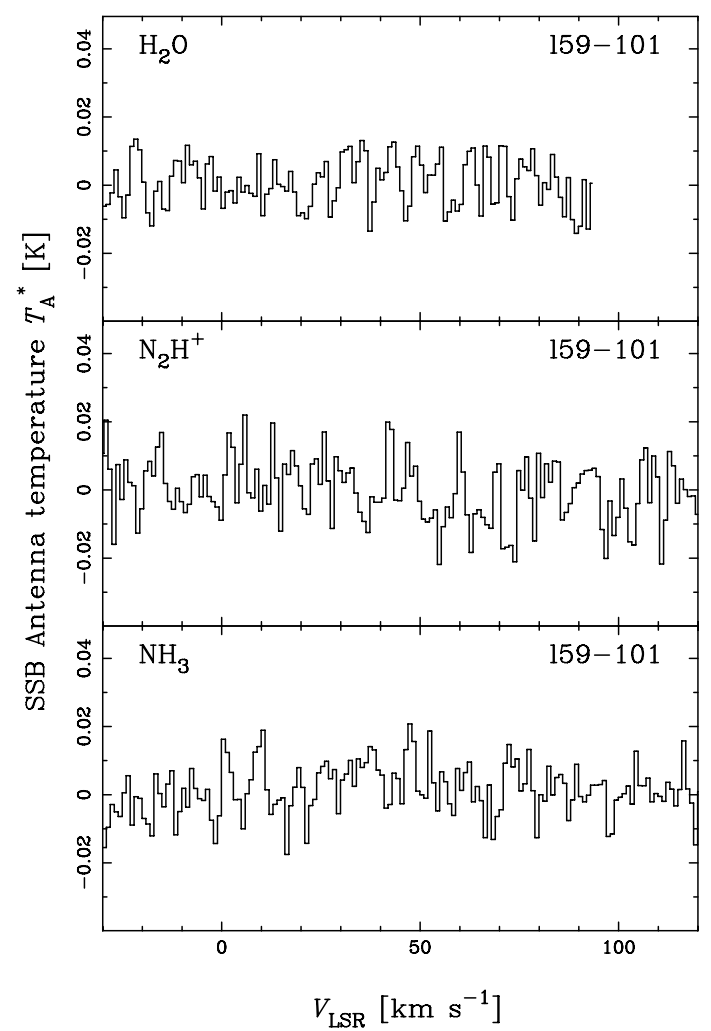

Fig. B.6.

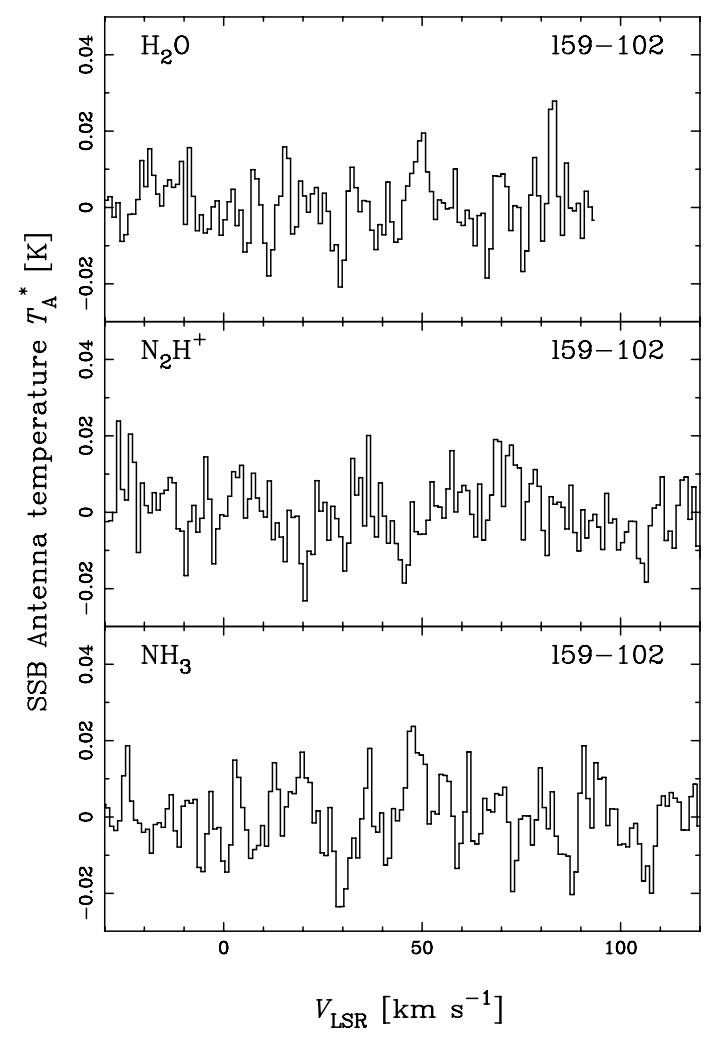

Fig. B.7. 


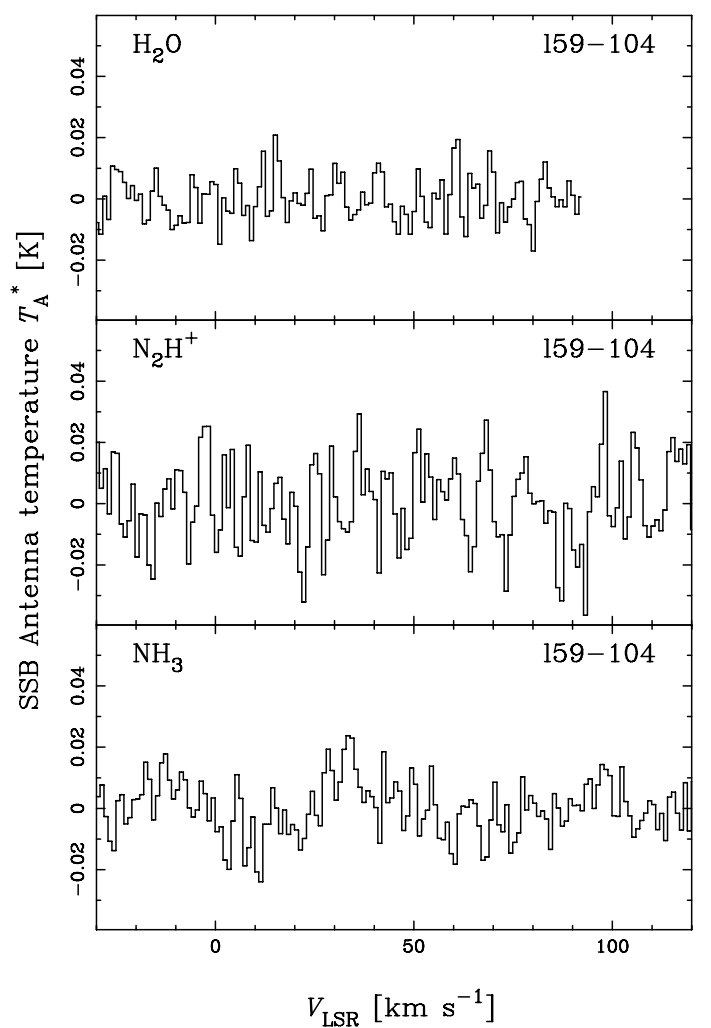

Fig. B.8.

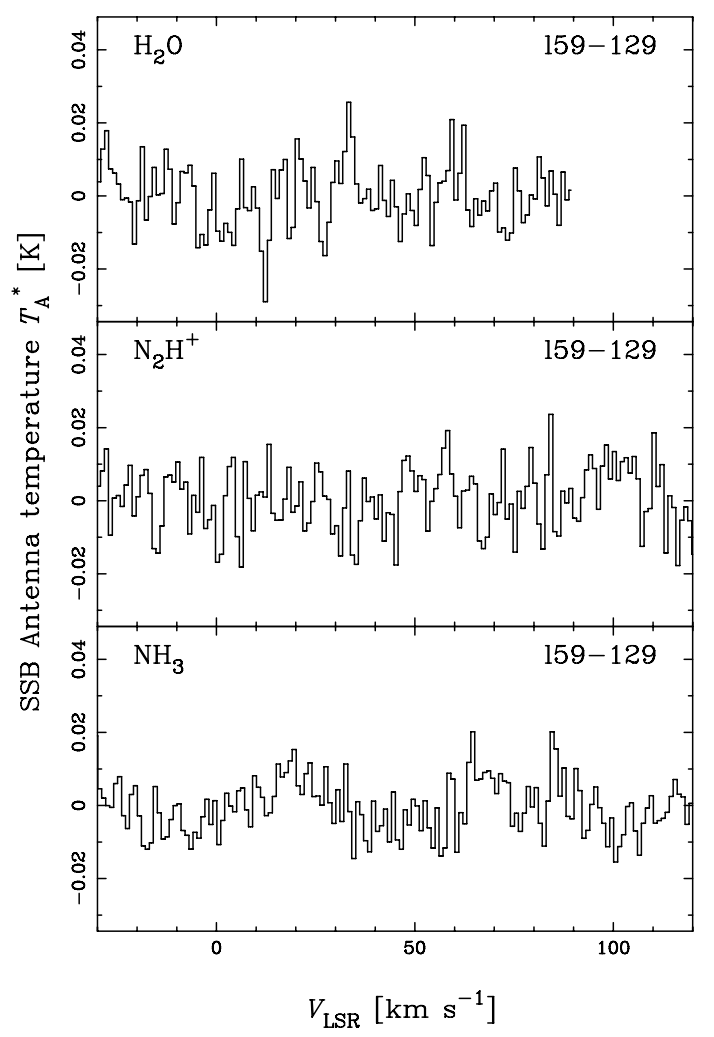

Fig. B.9.

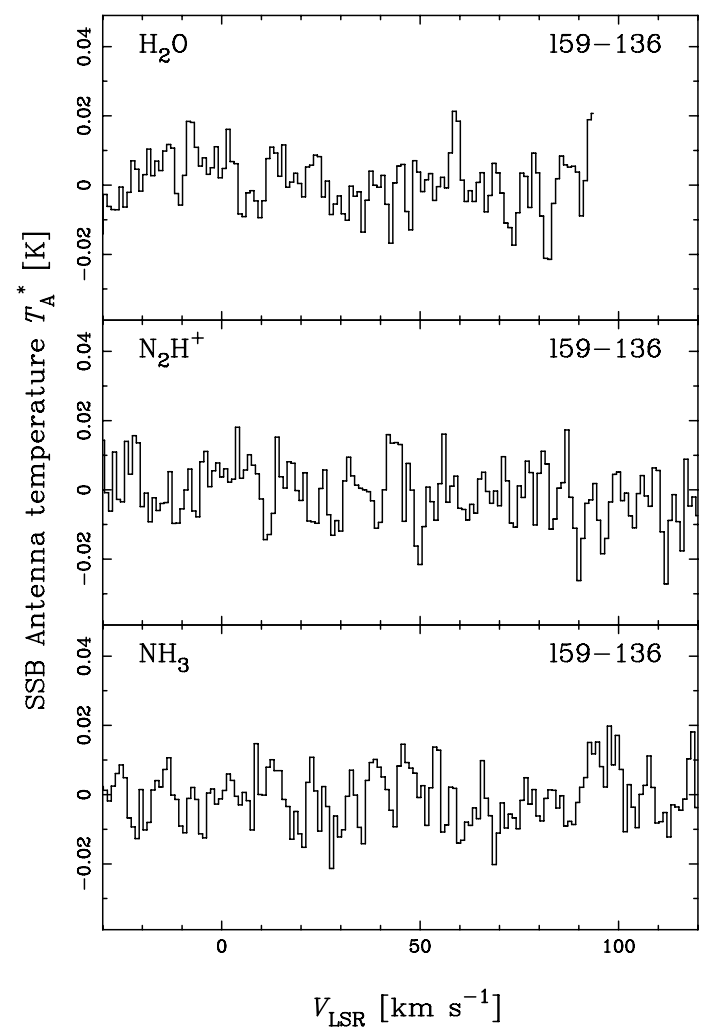

Fig. B.10.

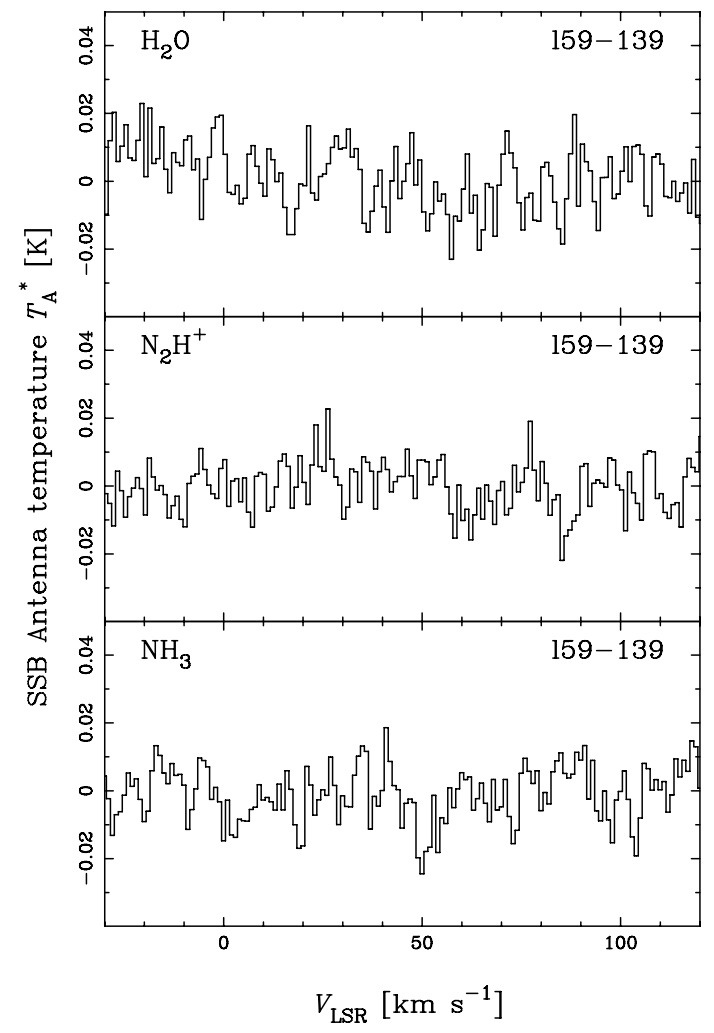

Fig. B.11. 
L. Olmi et al.: High-mass starless and protostellar clumps identified by the Hi-GAL survey

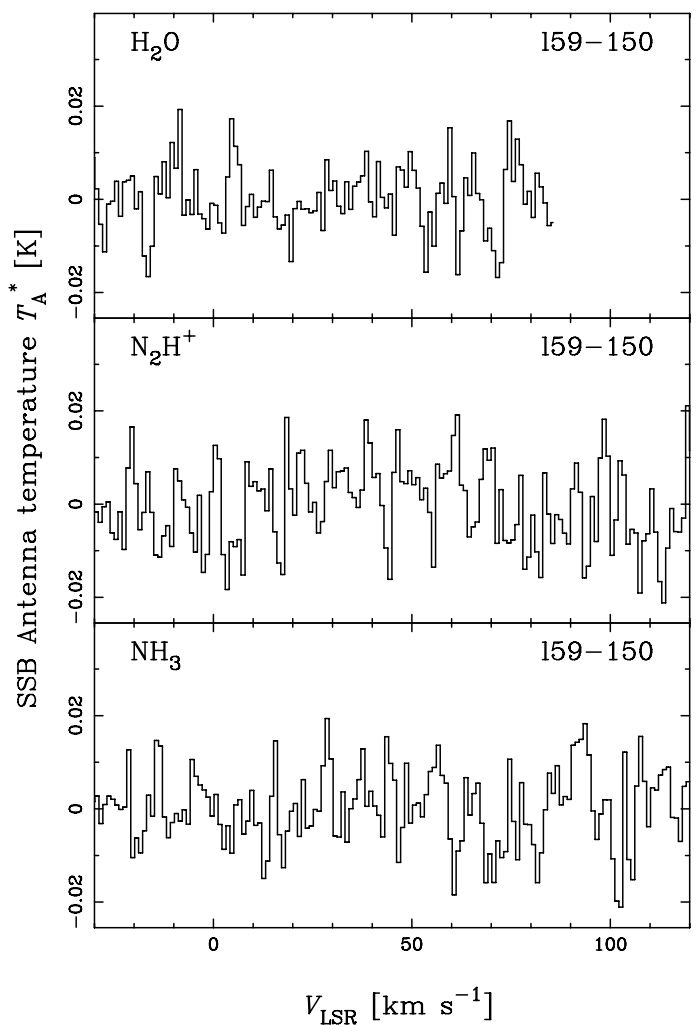

Fig. B.12.

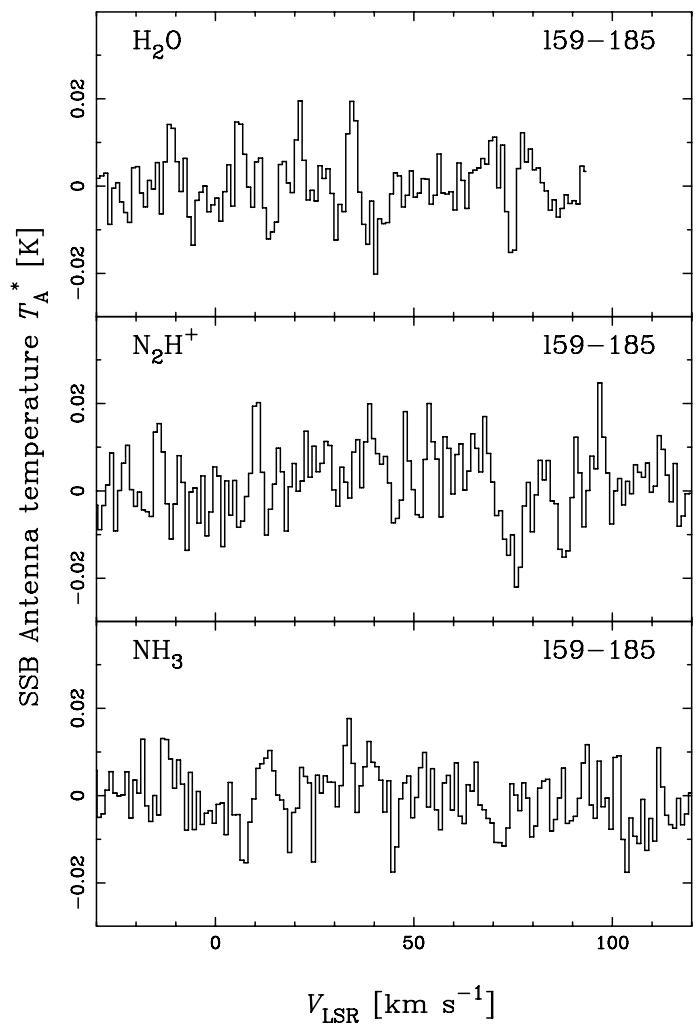

Fig. B.13.

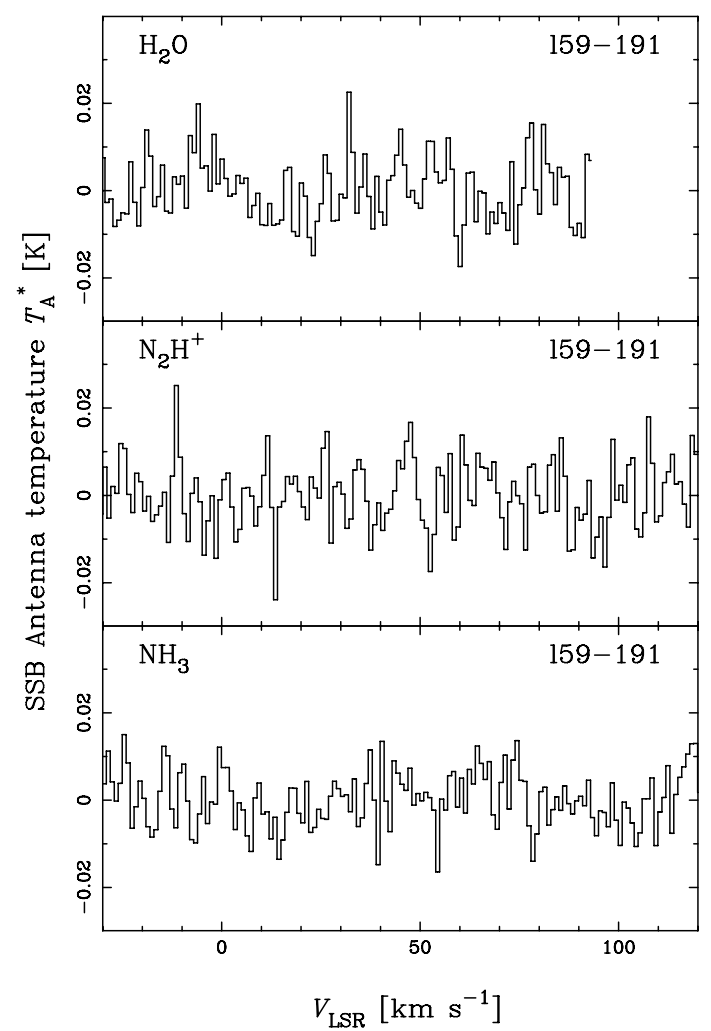

Fig. B.14.

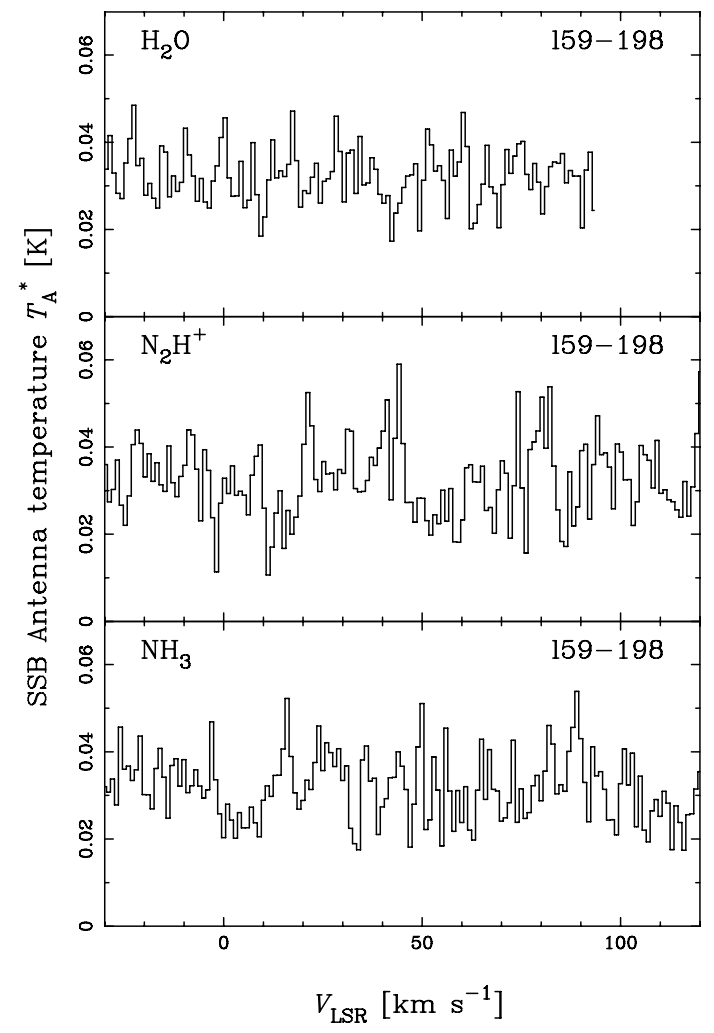

Fig. B.15. 


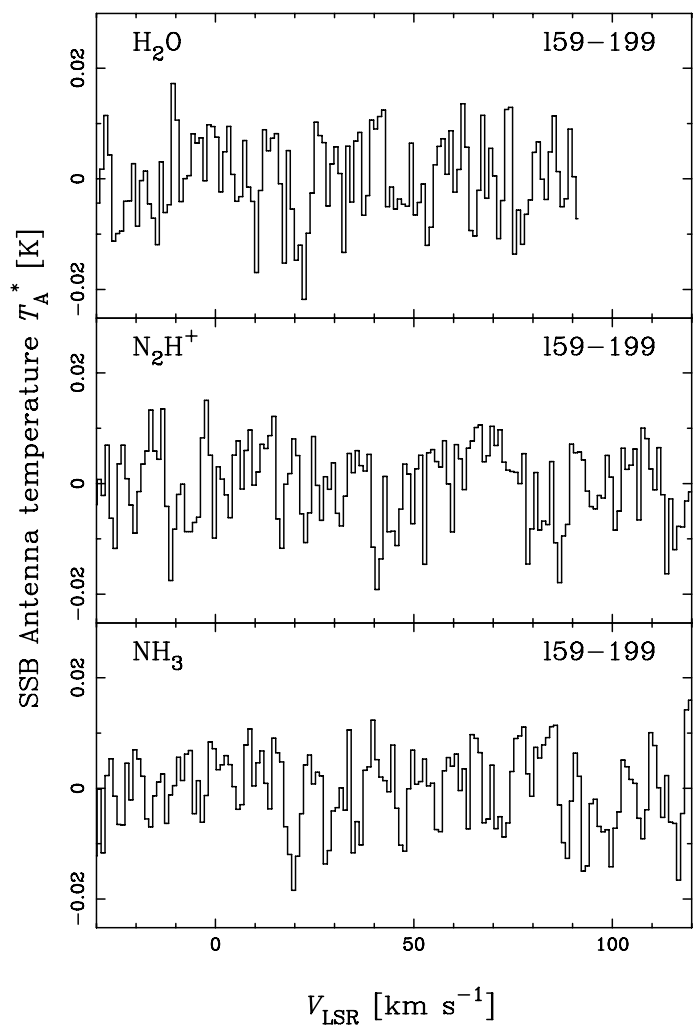

Fig. B.16.

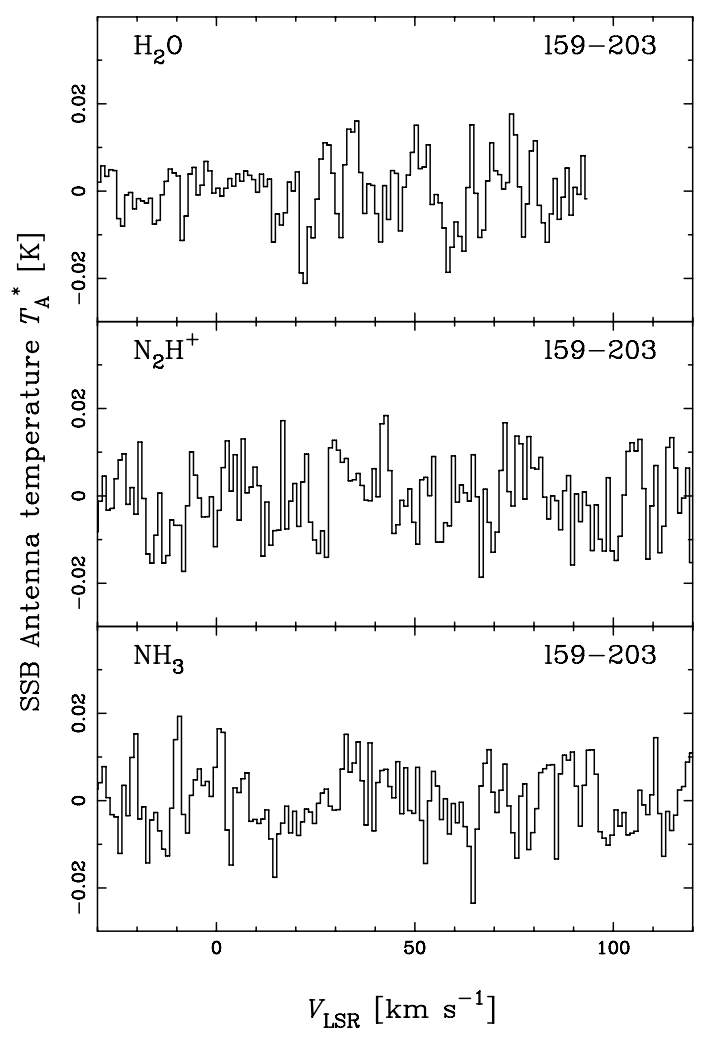

Fig. B.17.

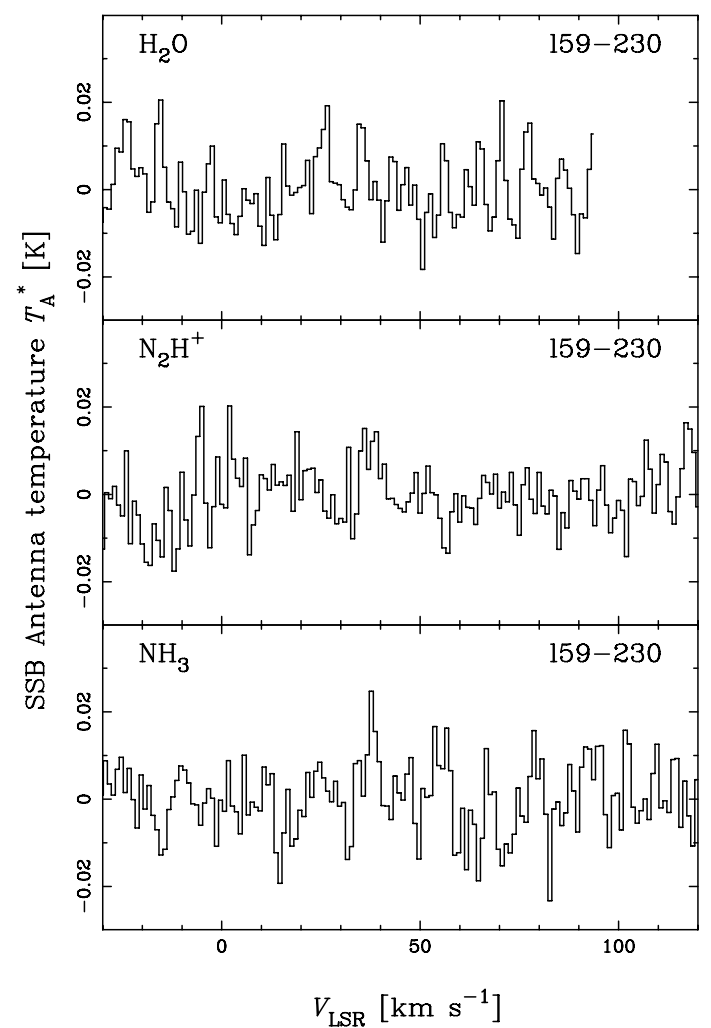

Fig. B.18.

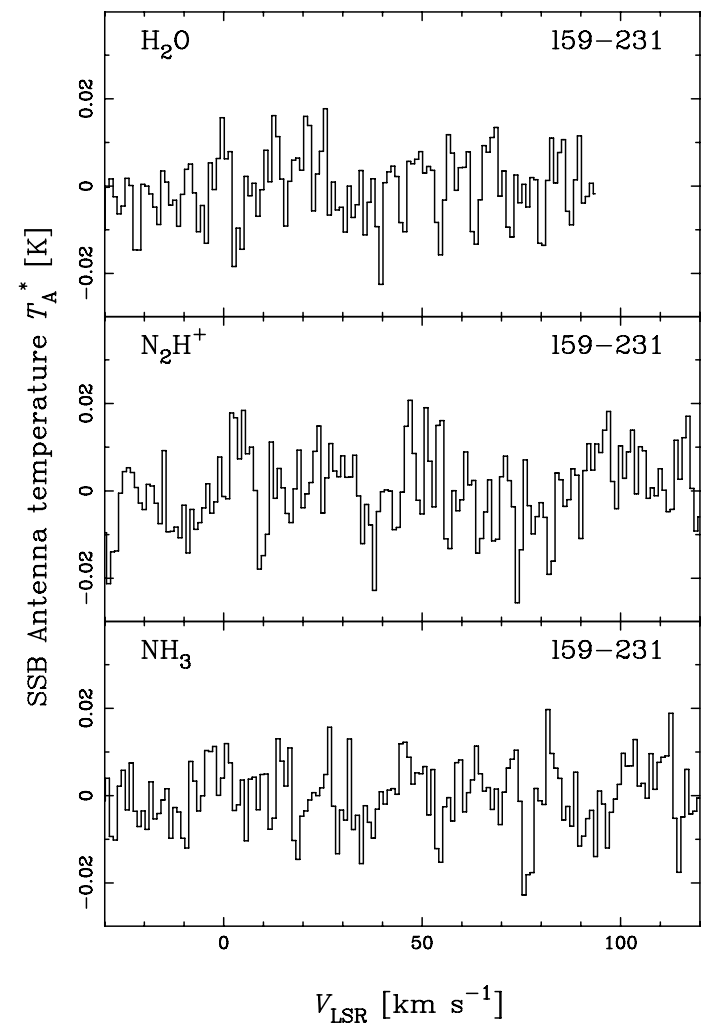

Fig. B.19. 
L. Olmi et al.: High-mass starless and protostellar clumps identified by the Hi-GAL survey

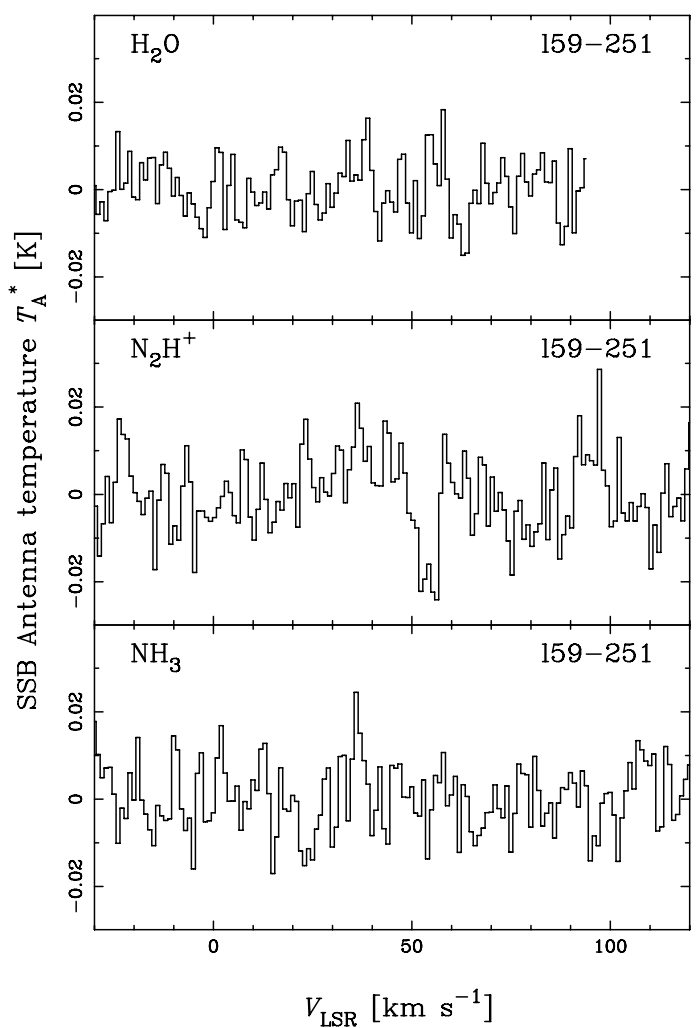

Fig. B.20.

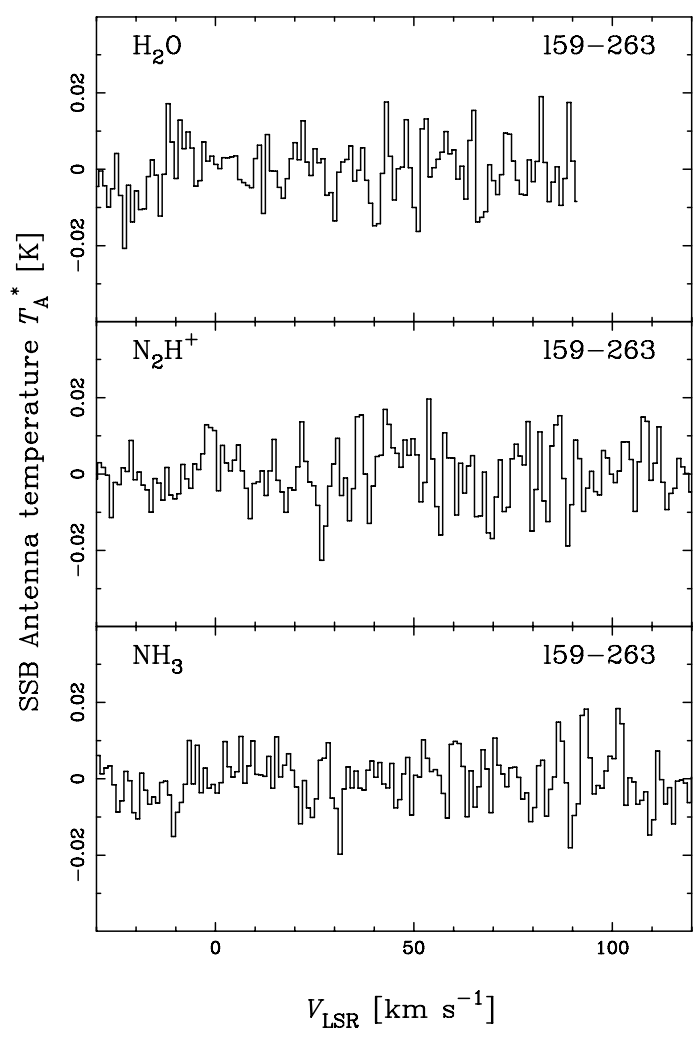

Fig. B.21.

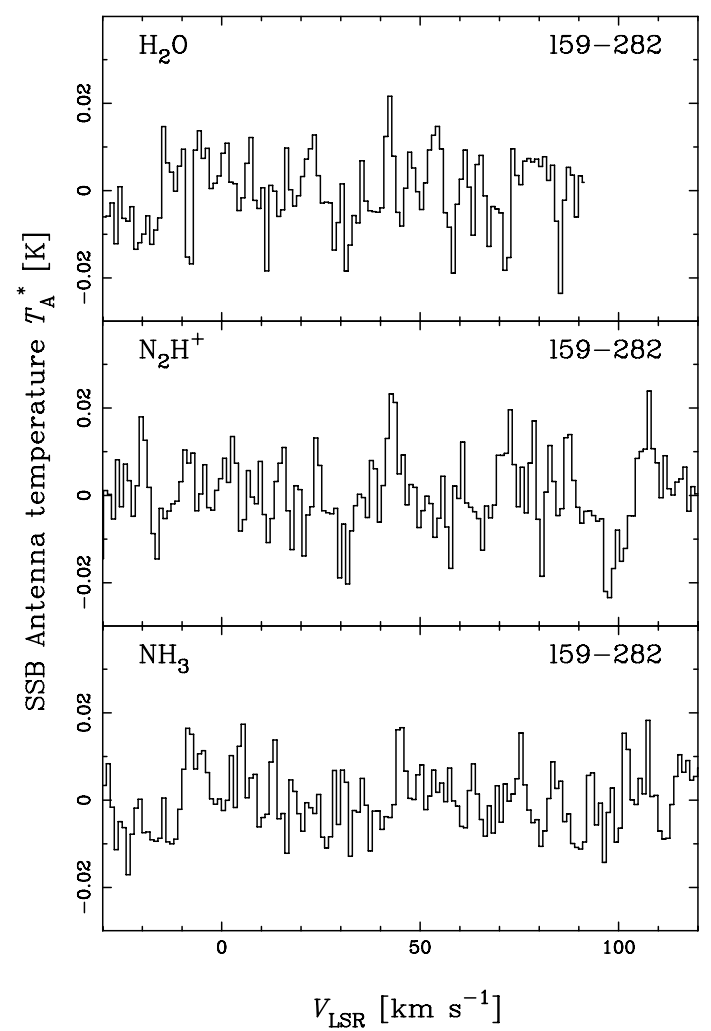

Fig. B.22.

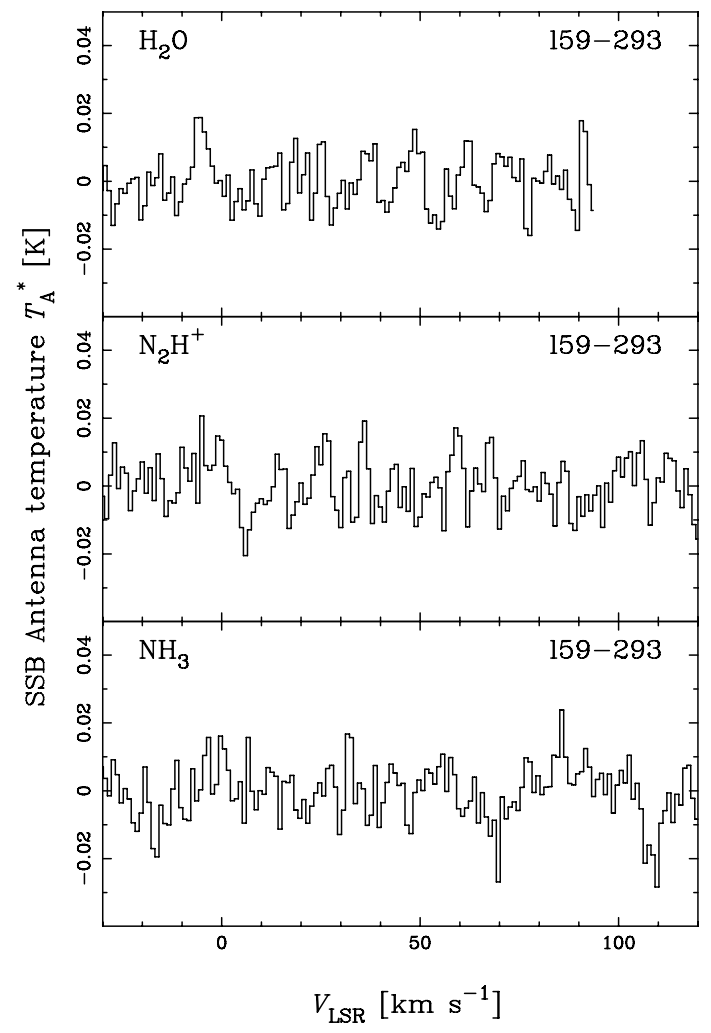

Fig. B.23. 


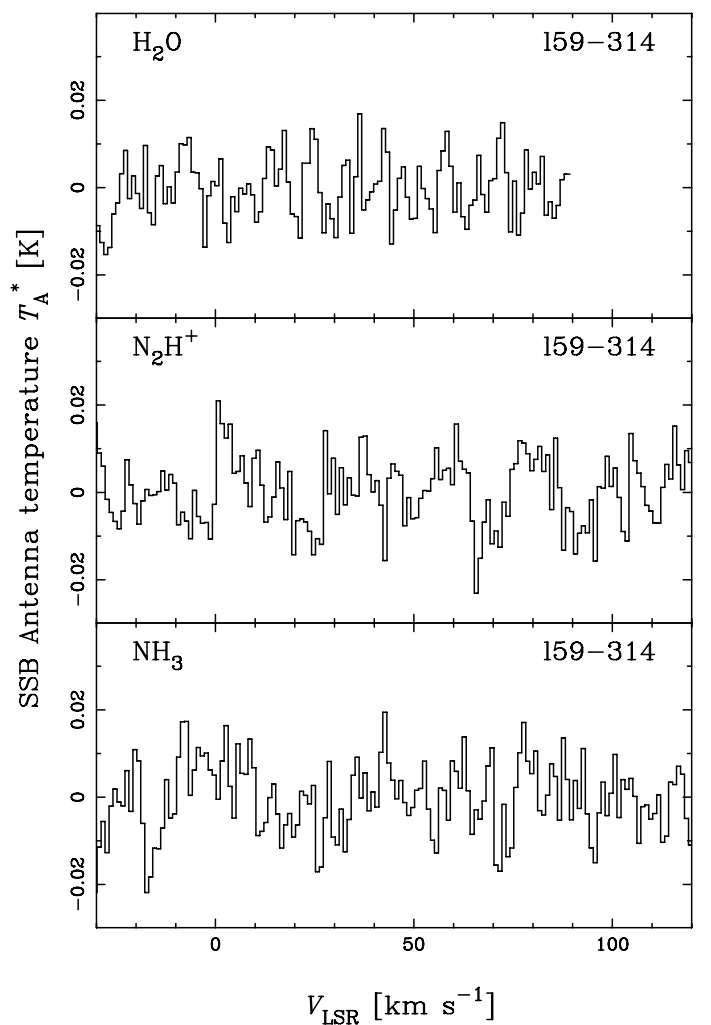

Fig. B.24.

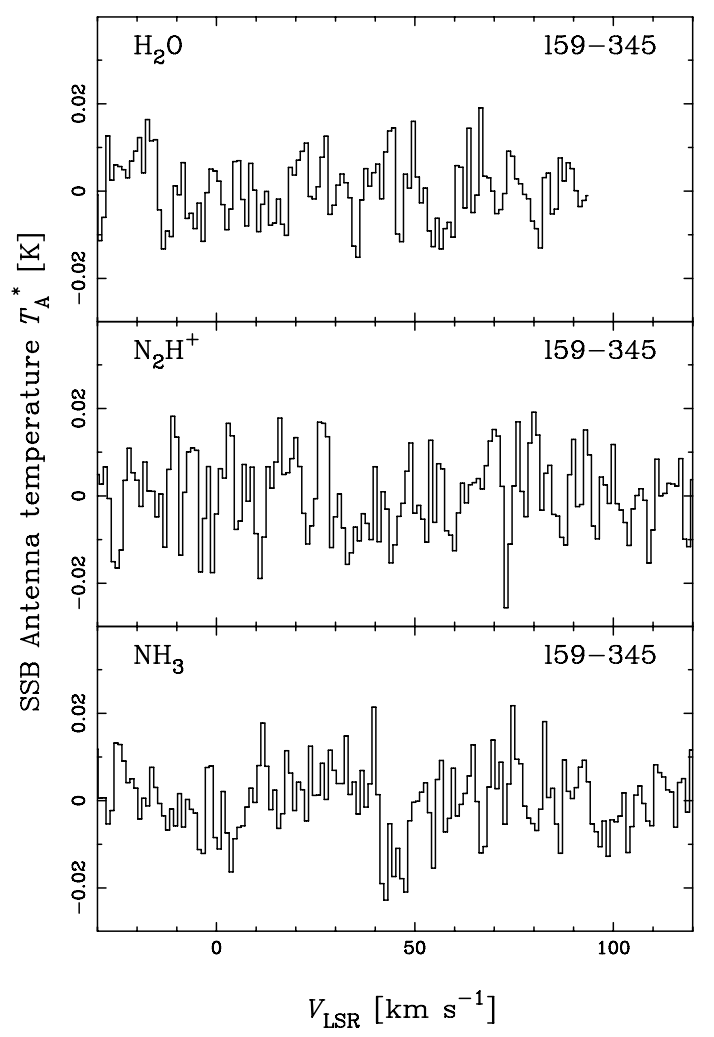

Fig. B.25.

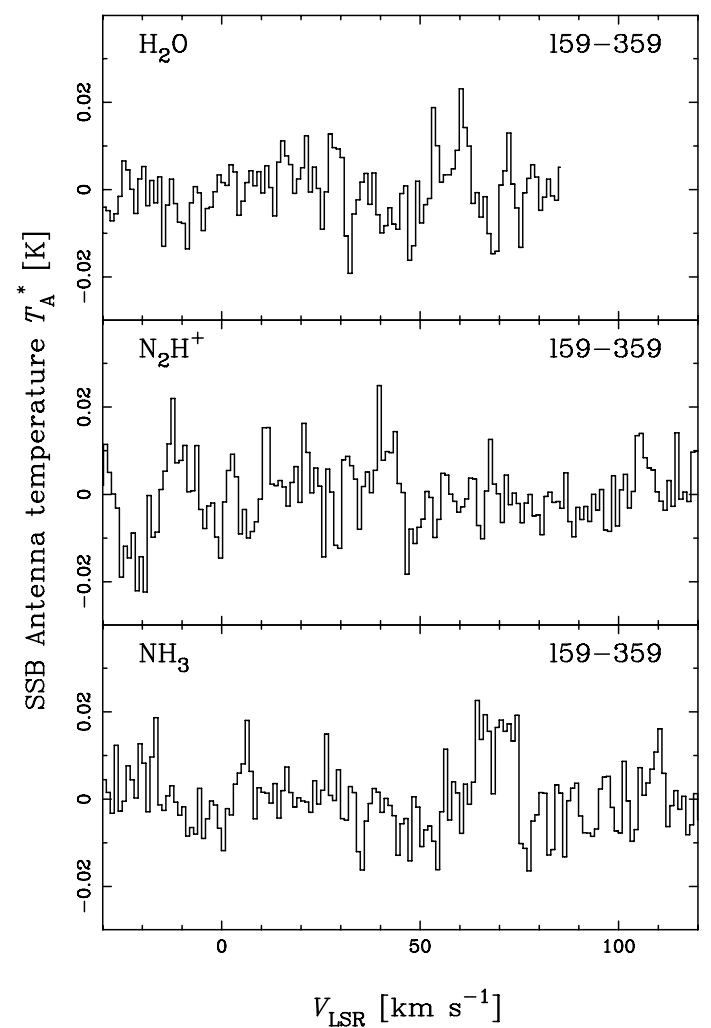

Fig. B.26.

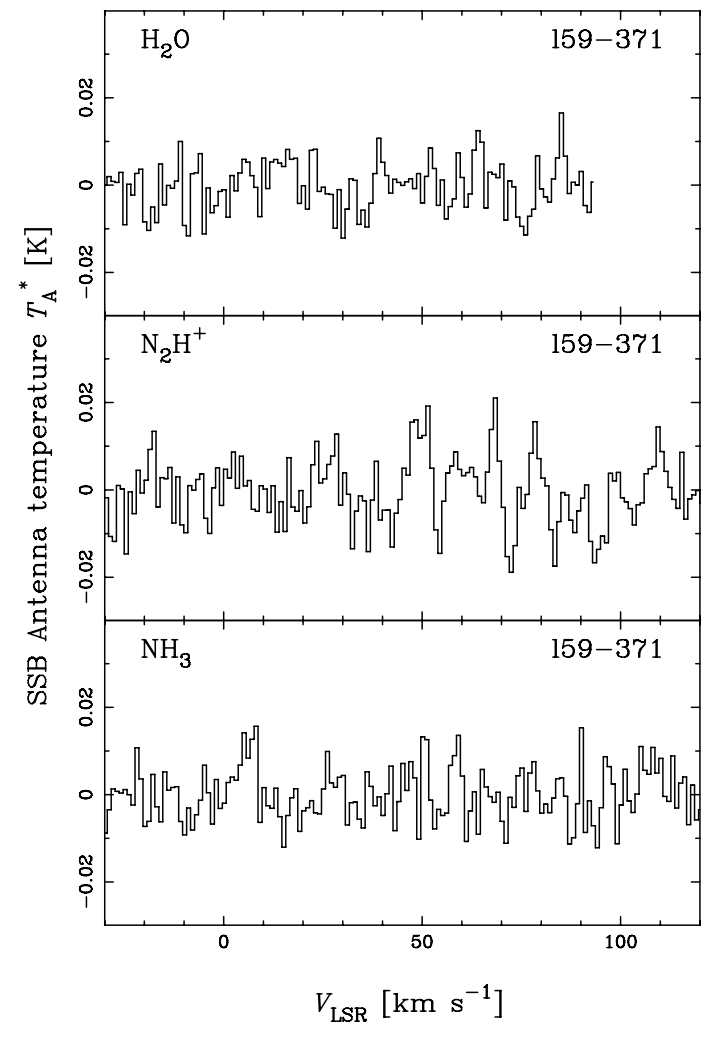

Fig. B.27. 
L. Olmi et al.: High-mass starless and protostellar clumps identified by the Hi-GAL survey

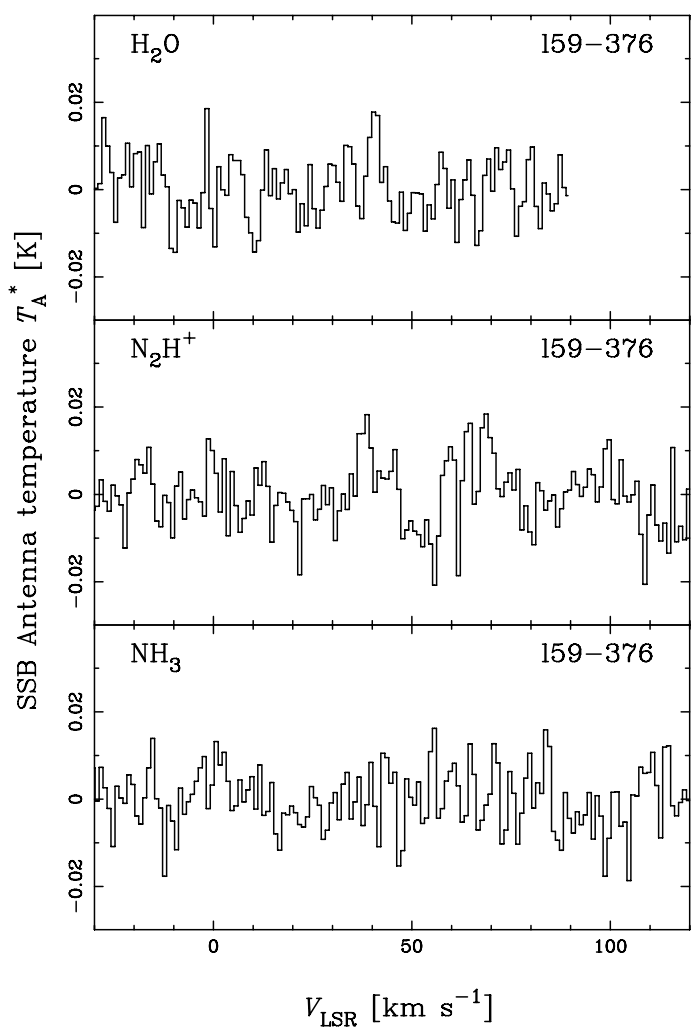

Fig. B.28.

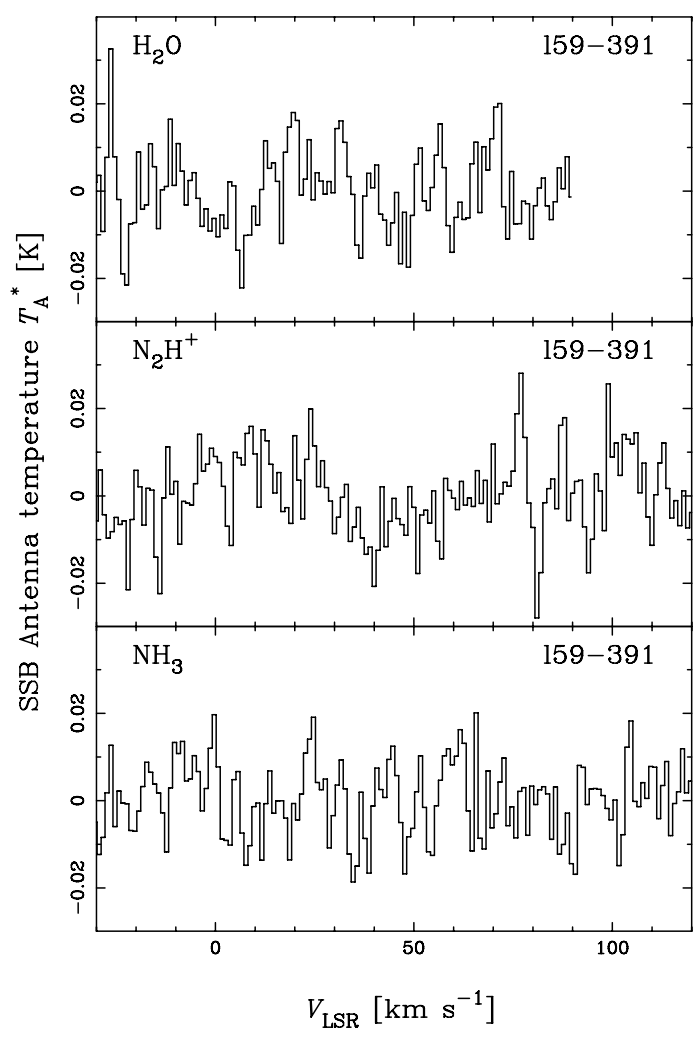

Fig. B.29.

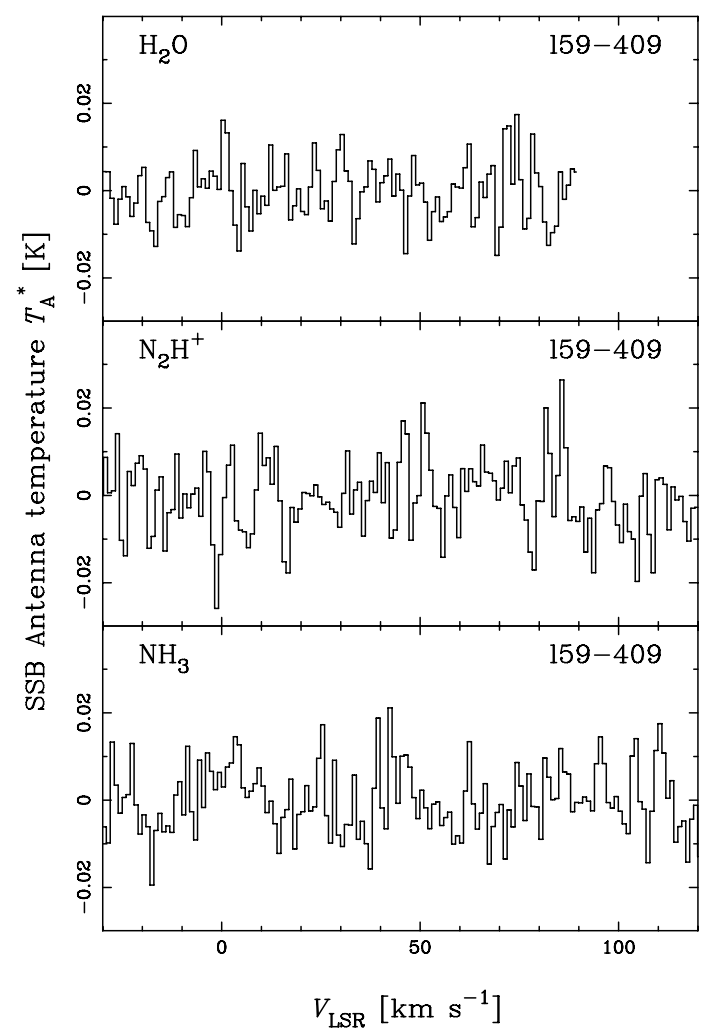

Fig. B.30.

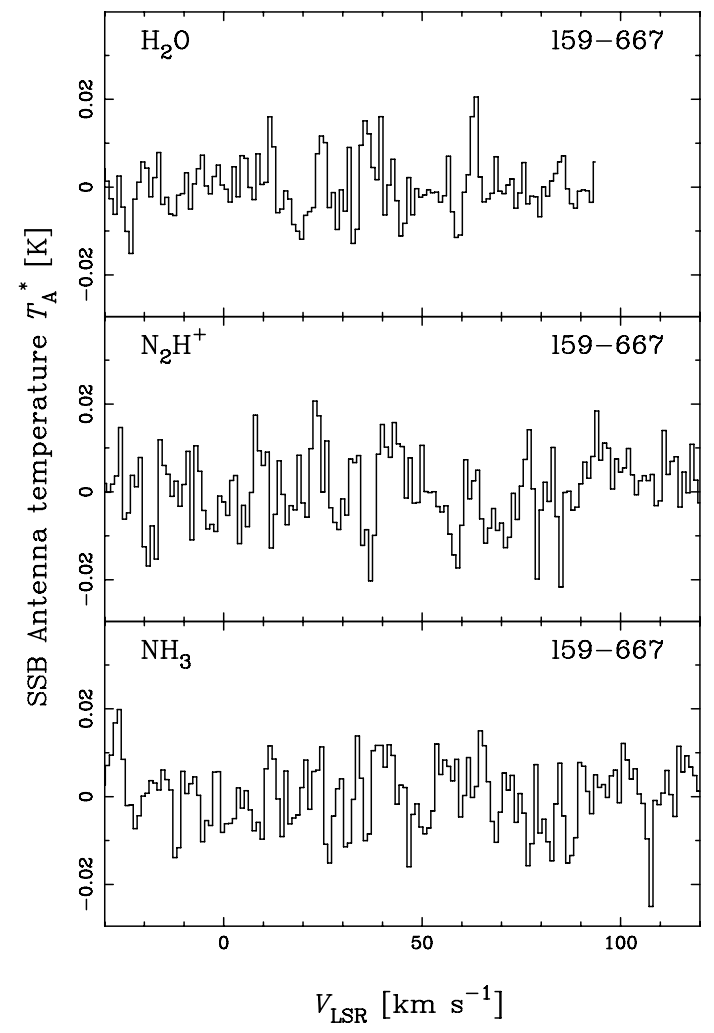

Fig. B.31. 


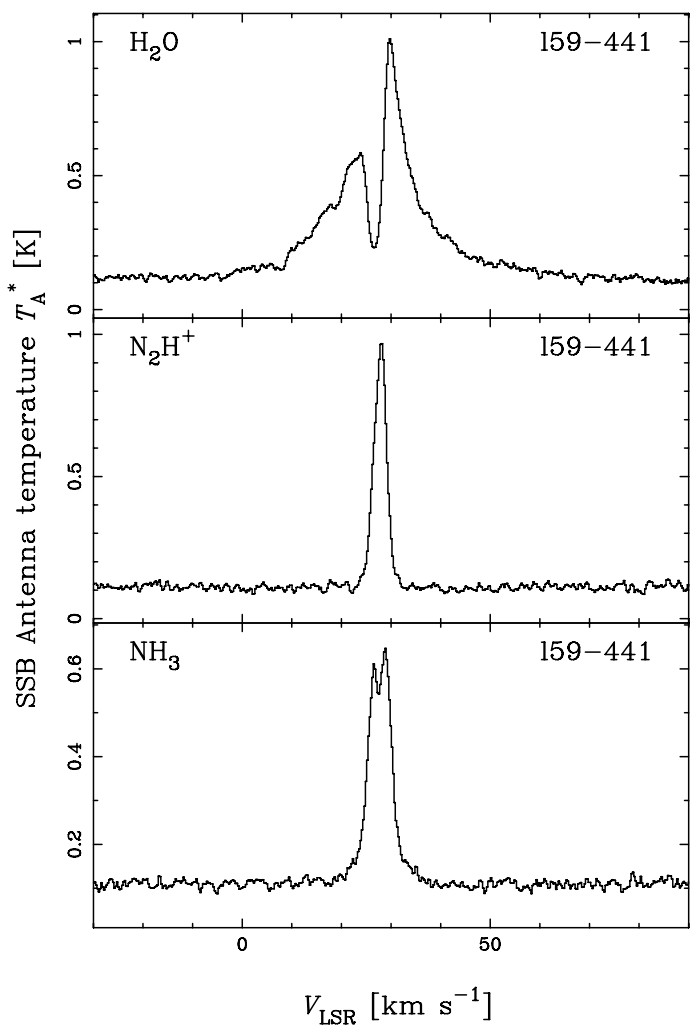

Fig. B.32. Original channel width $\left(0.27 \mathrm{~km} \mathrm{~s}^{-1}\right)$.

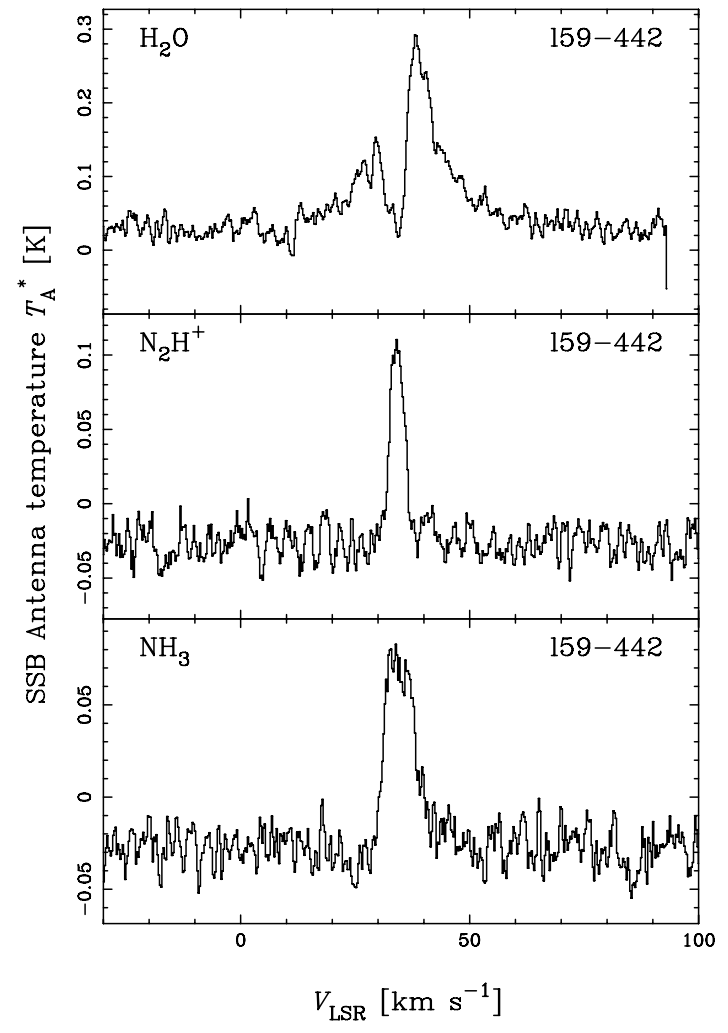

Fig. B.33. Original channel width $\left(0.27 \mathrm{~km} \mathrm{~s}^{-1}\right)$.

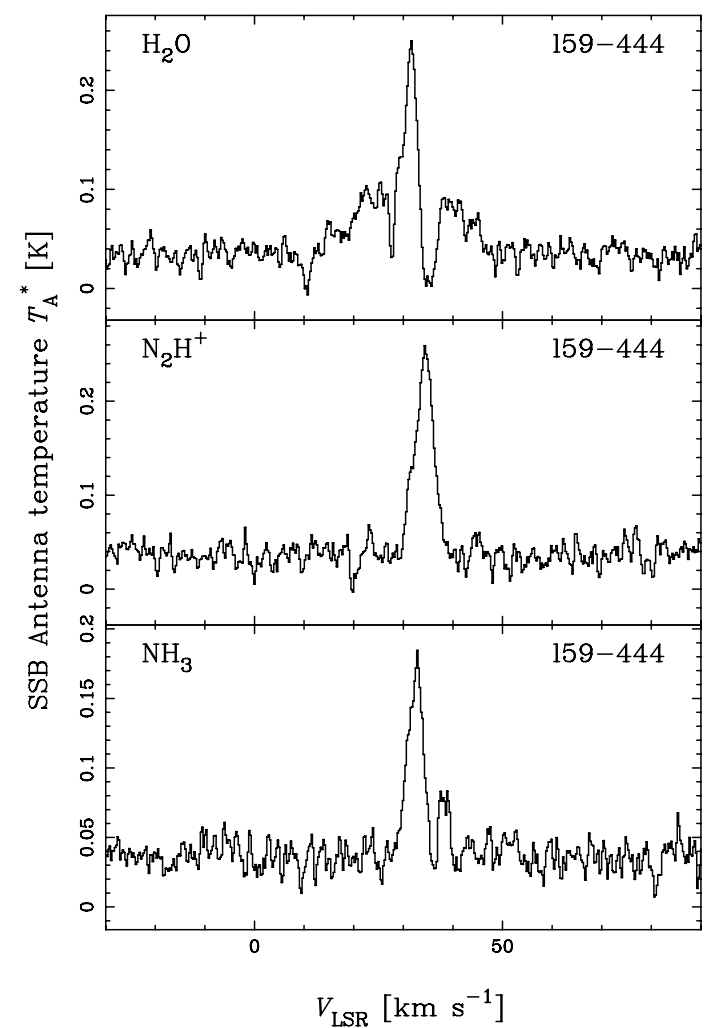

Fig. B.34. Original channel width $\left(0.27 \mathrm{~km} \mathrm{~s}^{-1}\right)$.

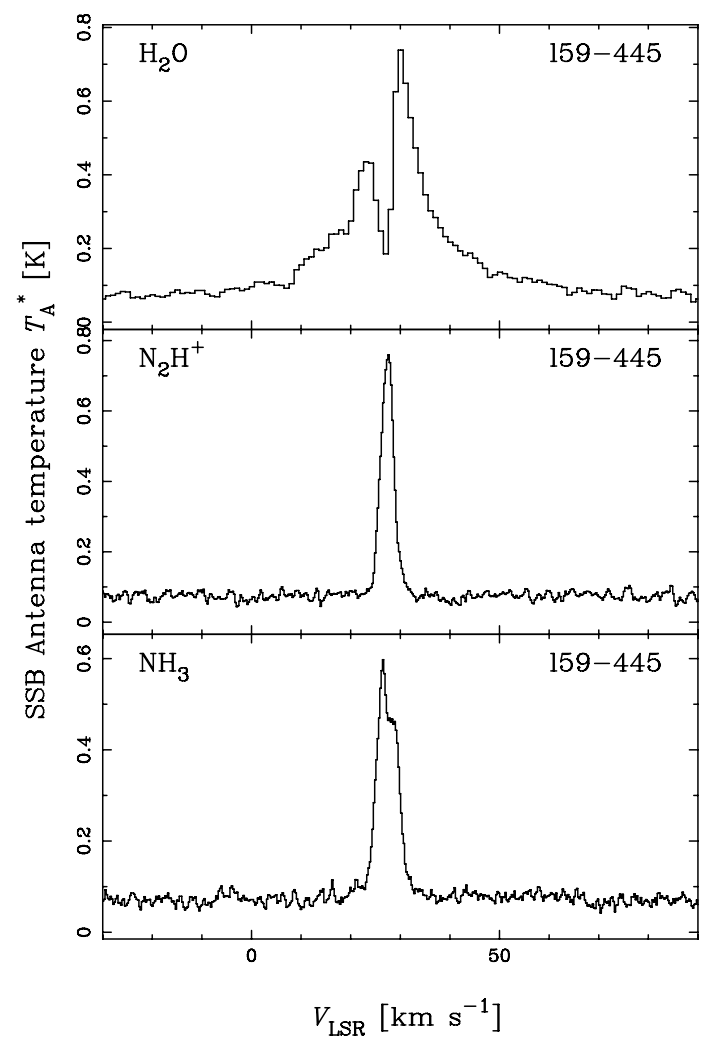

Fig. B.35. Original channel width $\left(0.27 \mathrm{~km} \mathrm{~s}^{-1}\right)$. 